\title{
Jets from massive unstable particles: Top-mass determination
}

\author{
Sean Fleming, ${ }^{1, *}$ Andre H. Hoang, ${ }^{2, \dagger}$ Sonny Mantry, ${ }^{3,}$ and Iain W. Stewart ${ }^{4, \S}$ \\ ${ }^{1}$ Department of Physics, University of Arizona, Tucson, Arizona 85721, USA \\ ${ }^{2}$ Max-Planck-Institut für Physik, Föhringer Ring 6, 80805 München, Germany \\ ${ }^{3}$ California Institute of Technology, Pasadena, California 91125, USA \\ ${ }^{4}$ Department of Physics, Massachusetts Institute of Technology, Boston, Massachusetts 02139, USA
}

(Received 9 April 2007; published 14 April 2008)

\begin{abstract}
We construct jet observables for energetic top quarks that can be used to determine a short-distance top quark mass from reconstruction in $e^{+} e^{-}$collisions with accuracy better than $\Lambda_{\mathrm{QCD}}$. Using a sequence of effective field theories we connect the production energy, mass, and top width scales, $Q \gg m \gg \Gamma$, for the top jet cross section, and derive a QCD factorization theorem for the top invariant mass spectrum. Our analysis accounts for $\alpha_{s}$ corrections from the production and mass scales, corrections due to constraints in defining invariant masses, nonperturbative corrections from the cross talk between the jets, and $\alpha_{s}$ corrections to the Breit-Wigner line shape. This paper mainly focuses on deriving the factorization theorem for hemisphere invariant mass distributions and other event shapes in $e^{+} e^{-}$collisions applicable at a future linear collider. We show that the invariant mass distribution is not a simple Breit-Wigner function involving the top width. Even at leading order it is shifted and broadened by nonperturbative soft QCD effects. We predict that the invariant mass peak position increases linearly with $Q / m$ due to these nonperturbative effects. They are encoded in terms of a universal soft function that also describes soft effects for massless dijet events. In a future paper we compute $\alpha_{s}$ corrections to the jet invariant mass spectrum, including a summation of large logarithms between the scales $Q, m$, and $\Gamma$.
\end{abstract}

DOI: 10.1103/PhysRevD.77.074010

PACS numbers: $14.65 . \mathrm{Ha}$

\section{INTRODUCTION}

Precise determinations of the top quark mass $m$ are among the most important standard model measurements being carried out at the Tevatron and being planned at the Large Hadron Collider (LHC) and a future International Linear Collider (ILC). A precise top-mass determination is important for precision electroweak constraints, as well as extensions to the standard model like minimal supersymmetry [1]. The present combined measurement from the Tevatron is $m=171.4 \pm 2.1 \mathrm{GeV}[2,3]$ and mainly relies on methods where a number of top-mass dependent kinematical quantities and observables are used in a global fit to determine the most likely top quark mass. For these fitting methods $[4,5]$ the observable most sensitive to the top quark mass is the top invariant mass distribution. It is obtained from reconstructing the total invariant mass of the top decay products. At the Tevatron the invariant mass distribution is being used in connection with other topmass-dependent observables due to the limited statistics.

In principle the reconstruction of the top invariant mass distribution provides the most natural way to measure the top quark mass since the peaked structure at the resonance is most closely related to the notion of the mass of a propagating massive and unstable degree of freedom.

\footnotetext{
*fleming@physics.arizona.edu

†ahoang@mppmu.mpg.de

mantry@theory.caltech.edu

§iains@mit.edu
}

This method can be applied at the LHC and ILC where larger statistics are available. Experimental studies have concluded that at the LHC top-mass measurements with uncertainties at the level of $1 \mathrm{GeV}[6,7]$ can be achieved, while at the ILC even smaller uncertainties can be expected $[8,9]$. However, since the top quark is a parton carrying nonvanishing color charge, its mass is a priori not directly observable. In fact the top mass should be considered as a renormalization scheme-dependent coupling in the QCD Lagrangian rather than a physical object, just like the strong coupling $\alpha_{s}$. As such, the top mass obtained from reconstruction also depends on the method and prescription that is used to define the top invariant mass since the latter is not a unique physical quantity. In fact the notion of a physical particle whose squared four-momentum is the mass does not apply to the top quark if one asks for a precision in the mass value that is comparable to the hadronic scale. This is also reflected in a number of conceptual and experimental issues for top quark mass determinations that are associated with gluon radiation, underlying events, and the jet energy scales-effects that can never be fully eliminated for measurements of the top quark mass from reconstruction. Moreover, certain top quark mass renormalization schemes are more suitable for precision measurements than others since the choice of scheme can affect the higher order behavior of the perturbative corrections as well as the organization of power corrections. Suitable quark mass schemes are compatible with the power counting and also lead to an optimal 
behavior of the perturbative expansion. Such schemes can be identified and defined unambiguously if the precise relation of the observable to a given Lagrangian top quark mass scheme can be established.

For all jet based methods of top quark mass determination, and for reconstruction, in particular, these issues have been intrinsically difficult to address in the past. Previous work has not provided a coherent analytic framework in which perturbative and nonperturbative effects could be described in a systematic manner. Considering the expected precision for top quark mass measurements in the upcoming experiments such a framework is imperative.

A top-mass determination method where a systematic analytic framework exists and where the relation between the Lagrangian top-mass parameter $m$ and the measured top mass can be established to high precision is the threshold scan of the line-shape of the total hadronic cross section in the top-antitop threshold region, $Q \approx 2 \mathrm{~m}$, at a future Linear Collider [10-14], where $Q$ is the c.m. energy. In this case the system of interest is a top-antitop quark pair in a color singlet state and the observable is related to a comparatively simple counting measurement. The lineshape of the cross section rises near a center of mass energy that is related to a toponiumlike top-antitop bound state with a mass that can be computed perturbatively to very high precision [15-19] using nonrelativistic QCD [20,21], an effective field theory (EFT) for nonrelativistic heavy quark pairs. The short lifetime of the top quark, $\tau=1 / \Gamma \approx$ $(1.5 \mathrm{GeV})^{-1}$, provides an infrared cutoff for all kinematic scales governing the top-antitop dynamics and leads to a strong power suppression of nonperturbative QCD effects. Experimental studies concluded that theoretical as well as experimental systematic uncertainties for this method are at a level of only $100 \mathrm{MeV}[22,23]$. The most suitable top quark mass schemes are the so-called threshold masses [15], which can be related accurately to other shortdistance mass schemes such as the running $\overline{\mathrm{MS}}$ mass. Unfortunately, the threshold scan method cannot be used at the LHC because the top-antitop invariant mass can only be determined with a relative uncertainty of around 5\% [24], which is not sufficient to resolve the top-antitop threshold region.

In this work we use EFT's to provide, for the first time, an analytic framework that can be applied to systematically describe the perturbative and nonperturbative aspects of top quark invariant mass distributions obtained from reconstruction. As a first step towards developing a detailed framework for the LHC, we focus in this work on jets in a $e^{+} e^{-}$linear collider environment at c.m. energies far above threshold $Q \sim 0.5--1 \mathrm{TeV}$. For $e^{+} e^{-}$collisions strong interaction effects arising from the initial state can be neglected and there is no need to identify or remove any "beam remnant" or underlying events. Also, in the $e^{+} e^{-}$ framework it is easier to formulate shape variables like thrust that control the jet-likeness and the soft dynamics of

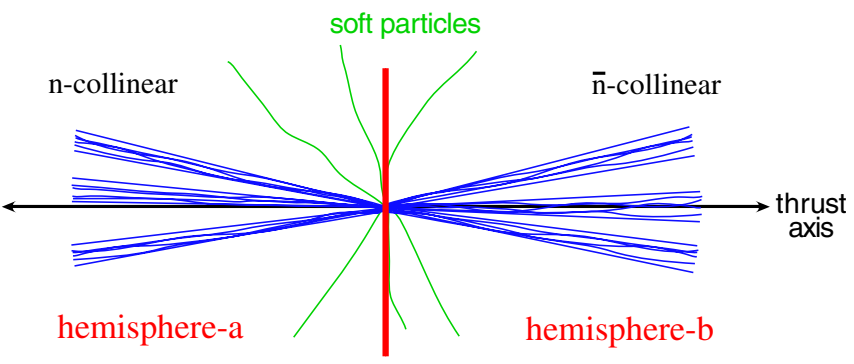

FIG. 1 (color online). Six jet event initiated by a top quark pair, $t \bar{t} \rightarrow b W \bar{b} W \rightarrow b q q^{\prime} \bar{b} q q^{\prime}$. The plane separating the two hemispheres is perpendicular to the thrust axis and intersects the thrust axis at the interaction point. The total invariant mass inside each hemisphere is measured. Our analysis applies equally well to the lepton + jets and the dilepton channels (not shown).

an event. We consider the double differential top and antitop invariant mass distribution, where each of the invariant masses, $M_{t}^{2}$ and $M_{\bar{t}}^{2}$, are defined from all particles in each of the two hemispheres that are determined by the event's thrust axis. In Fig. 1 we show a sketch of such an event. Other invariant mass definitions, e.g. based on $k_{T}$ algorithms and criteria to identify jets from top and antitop decay can be employed as well. Our approach also works for all-jet and lepton-plus-jet final states. Our focus is to study the double differential invariant mass distribution in the peak region close to the top mass, so that $M_{t}^{2}-m^{2} \sim$ $m \Gamma$ and $M_{\bar{t}}^{2}-m^{2} \sim m \Gamma$. It is convenient to introduce the shifted variables

$$
\hat{s}_{t, \bar{t}} \equiv \frac{s_{t, \bar{t}}}{m} \equiv \frac{M_{t, \bar{t}}^{2}-m^{2}}{m} \sim \Gamma \ll m,
$$

because it is only the invariant mass distribution close to the peak that we wish to predict. Here the top width $\Gamma$ is setting a lower bound on the width of the invariant mass distribution and the shifted variable $\hat{s}_{t, \bar{t}}$ can also be larger than $\Gamma$ as long as $\hat{s}_{t, \bar{t}} \ll m$. However, for simplicity we will often write $\hat{s}_{t, \bar{t}} \sim \Gamma$ as we did in Eq. (1).

There are three relevant disparate scales governing the dynamics of the system,

$$
Q \gg m \gg \Gamma>\Lambda_{\mathrm{QCD}}
$$

This kinematic situation is characterized by energy deposits contained predominantly in two back-to-back regions of the detector with opening angles of order $m / Q$ associated with the energetic jets coming from the top quark decay and collinear radiation. Frequently in this work we refer to the jets coming from the top and antitop quark collectively as top and antitop jet, respectively, but we stress that we do not require the jets from the top and antitop decay products to be unresolved as pictured in Fig. 1 (for example one can still identify a $W$ and do $b$-tagging). The region between the top jets is predominantly populated by soft particles with energies of order of the hadronic scale. 


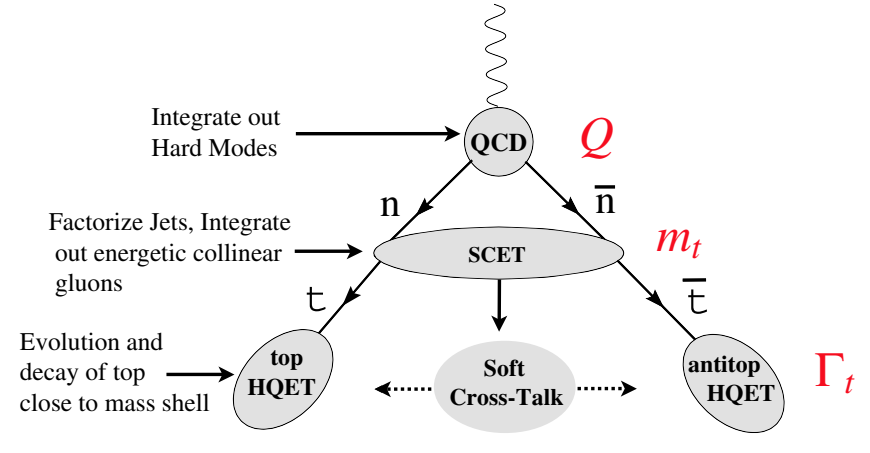

FIG. 2 (color online). Sequence of effective field theories used to compute the top/antitop invariant mass distribution in the peak region.

The EFT setup used to describe the dynamics in this kinematic situation is illustrated in Fig. 2 and represents a sequence of different EFT's. The use of different EFT's is mandatory to separate the various relevant physical fluctuations. The high energy dynamics for the top quarks at the scale $Q \gg m$ can be described by quark and gluon degrees of freedom that are collinear to the top and antitop jet axes, and by soft degrees of freedom that can freely propagate between the jets. The appropriate EFT for this situation is the soft collinear effective theory (SCET) [25-28] with a nonzero top quark mass term [29], which represents an expansion in $\lambda \sim m / Q \sim 0.2-0.3$. The leading order soft collinear decoupling [27] properties of SCET allows a factorization of the process into three sectors: top jet dynamics, antitop jet dynamics, and dynamics of the soft cross talk between the top and antitop jets, which corresponds quite intuitively to the situation pictured in Fig. 1. In SCET the typical fluctuation of the jet invariant masses around the top mass are still of order $m, \hat{s}_{t, \bar{t}} \sim m$. Thus to describe invariant masses in the peak region $\hat{s}_{t, \bar{t}} \sim \Gamma$ the top and antitop jets are finally computed in heavy quark effective theory (HQET) [30] which represents an expansion in $\hat{s} / m$ and $\Gamma / m \sim 0.01$. We have in fact two copies of HQET, one for the top and one for the antitop, plus soft interactions between them. In these EFT's the top decay can be treated as inclusive and is therefore described by the total top width term $\Gamma$ that acts as an imaginary residual mass term [10,31]. Since HQET is usually understood as being formulated close to the rest frame of the heavy quark without the soft cross talk interactions, we refer to these two EFT's as boosted HQET's (bHQET's). ${ }^{1}$

At leading order in the expansion in $m / Q$ and $\Gamma / m$ we show that the double differential invariant hemisphere mass distribution can be factorized in the form

\footnotetext{
${ }^{1}$ We adopt the acronym bHQET in cases where we wish to remind the reader that the residual momentum components of the heavy quark in the $e^{+} e^{-}$c.m. frame are not homogeneous, and that additional gluon interactions occur which are not simply the soft gluons of standard HQET.
}

$$
\begin{aligned}
\left(\frac{d \sigma}{d M_{t}^{2} d M_{\bar{t}}^{2}}\right)_{\text {hemi }}= & \sigma_{0} H_{Q}\left(Q, \mu_{Q}, \mu_{m}\right) H_{m}\left(m, \frac{Q}{m}, \mu_{m}, \mu\right) \\
& \times \int d \ell^{+} d \ell^{-} B_{+}\left(\hat{s}_{t}-\frac{Q \ell^{+}}{m}, \Gamma, \mu\right) \\
& \times B_{-}\left(\hat{s}_{\bar{t}}-\frac{Q \ell^{-}}{m}, \Gamma, \mu\right) S_{\text {hemi }}\left(\ell^{+}, \ell^{-}, \mu\right),
\end{aligned}
$$

where $\hat{s}_{t}$ and $\hat{s}_{\bar{t}}$ are defined in terms of $M_{t, \bar{t}}^{2}$ in Eq. (1). The term $\sigma_{0}$ is a normalization factor, and the factors $H_{Q}$ and $H_{m}$ are matching corrections that are derived from matching and running in SCET and the bHQET's, respectively. $H_{Q}$ and $H_{m}$ are independent of $\hat{s}_{t}$ and $\hat{s}_{\bar{t}}$ and do not affect the form of the invariant mass distributions. The jet functions $B_{ \pm}$describe the QCD dynamics of collinear radiation in the top/antitop direction, and the decay of the top and antitop quarks near mass shell within the top/antitop jets. They can be computed perturbatively at the scale $\mu \gtrsim \Gamma$ since the top width $\Gamma$ provides an infrared cutoff from hadronization. At tree level they are Breit-Wigner functions

$$
B_{ \pm}(\hat{s}, \Gamma)=\frac{1}{\pi m} \frac{\Gamma}{\hat{s}^{2}+\Gamma^{2}}+\ldots,
$$

where the ellipses indicate QCD corrections that distort the Breit-Wigner functions. For the computation of the $B_{ \pm}$it is mandatory to employ properly defined short-distance top mass schemes, to achieve a well-behaved perturbative expansion. Finally, the soft function $S_{\text {hemi }}\left(\ell^{+}, \ell^{-}\right)$describes the physics of the soft nonperturbative gluons through which the top and antitop jets can communicate. The low energy fluctuations of these soft gluons are not cut off by the large top quark width. This can be intuitively understood due to the lifetime dilation of the boosted top quarks. As explained in Sec. II, using soft collinear factorization, we can show that the soft function is universal, namely, that the same function governs the low energy dynamics for massless jets in the dijet limit [32-36]. So, information on the form of $S_{\text {hemi }}\left(\ell^{+}, \ell^{-}\right)$can be gained in a model-independent way from experimental data on massless dijet events. The form of the factorization theorem in Eq. (3) is based on the same principles as the factorization formula for massless dijet event shapes [32-34,36], but it differs due to the need to treat massive quark jets and effects related to the large top quark width. We also use our results to derive a factorization theorem for thrust and the heavy jet mass event shape for $t \bar{t}$ production in the peak region. These distributions can also be used to measure the top quark mass.

The convolution in Eq. (3) shows that the observed hemisphere mass distributions are inevitably distorted by the nonperturbative soft momentum distribution, and that top and antitop jets can only interact indirectly through exchange of different light-cone momentum components 
that are governed by the soft function. We can also show that for invariant masses $M_{t, \bar{t}}$ that are defined through the identification of the jets from top and antitop decay, which are determined from a $k_{T}$ jet algorithm, the same factorization formula as in Eq. (3) can be derived up to a different soft function.

We believe that the factorization approach proposed in this work, and the factorization formula in Eq. (3), represent advancements concerning the following points:

(i) We give a well-defined relation between a jet observable sensitive to the top mass and the Lagrangian mass. This allows the definition of a short-distance top mass which we call the "jet mass." Theoretically the jet mass can be determined with a precision better than $\Lambda_{\mathrm{QCD}}$, once the soft function governing nonperturbative effects is known by other means. We expect that the jet mass will be useful for a broad range of observables involving jets and parton showering from massive quarks.

(ii) The soft function appearing in the massive-jet factorization formula is universal, and appears in massless dijet event shapes. This universality can reduce the dependence of uncertainties in the top mass from reconstruction on parton shower Monte Carlos and hadronization models.

(iii) The factorization approach opens up the possibility to systematically construct top-mass observables where nonperturbative effects are suppressed.

While the focus of this paper is on $t \bar{t}$ production at an $e^{+} e^{-}$ linear collider, the main ideas and tools developed are general, and will also play an important role for the environment of the LHC where a substantial number of top events with large $p_{T}$ will be available. Other applications of our approach to factorization of jets from massive particles may include processes such as single top production [37,38], W-pair production, or processes involving new colored unstable particles [39,40]. We briefly comment on these applications in the summary.

The outline of the paper is as follows. In Sec. II we describe the relevant EFT formalism for our computation. In Sec. III we derive the factorization theorem in SCET, introduce the hemisphere jet invariant masses, and perform the factorization of mass effects in boosted HQET. The result of the analysis in this section is the complete factorization theorem for the double invariant mass distribution, and the extension to thrust and the heavy jet mass event shapes. In this section we also define the short-distance jet mass scheme. In Sec. IV we study the factorization theorem numerically at leading order and discuss implications for top-mass measurements. We also display numerical results for the shape of the peak region. In Sec. V we discuss the relation between the factorization theorem for the hemisphere invariant masses used in our work, to a factorization theorem for the reconstruction method based on $k_{T}$ jet algorithms employed in Refs. [8,9]. Finally we summarize and conclude in Sec. VI.
This paper concentrates on the derivation of the factorization theorem, on field theoretic issues, and on the basic phenomenological implications of our result. Readers only interested in the final result may skip over the analysis in Secs. II and III, and go directly to Sec. IV. In a future paper we present the computation of $\alpha_{s}$ corrections to the jet invariant mass cross section, and the summation of large logarithms between the scales $Q, m, \Gamma$.

\section{THE EFFECTIVE FIELD THEORIES}

In this section we discuss the EFT's required to compute the double differential invariant mass distribution $d^{2} \sigma / d M_{t}^{2} d M_{\bar{t}}^{2}$ in the peak region. The relevant energy scales are

$$
Q \gg m \gg \hat{s}_{t} \sim \hat{s}_{\bar{t}} \sim \Gamma,
$$

where the hatted $s$ variables were defined in terms of $M_{t}^{2}$ and $M_{\bar{t}}^{2}$ in Eq. (1). Once radiative corrections are included, large logarithms arise through ratios of the above energy scales, some of which are double logs, and thus can be quite large. For example $\Gamma / m \approx 1 / 120$, so $\ln ^{2}(\Gamma / m) \approx$ 25 . It is obviously important to understand the appearance of all large logs as accurately as possible, and to sum them systematically. This summation is accomplished by matching onto a sequence of EFTs and using renormalization group equations.

Starting from QCD we first switch to the SCET [26$28,41]$ for massive quarks and then to heavy quark effective theory [42-46] combined with the unstable particle EFT method [31,47-49]. This scheme includes systematically effects related to the large top quark width, as well as interactions related to the soft cross talk:

$$
\begin{aligned}
\text { QCD } & \rightarrow \text { SCET } \\
& \rightarrow \text { boosted-HQET with unstable heavy quarks. }
\end{aligned}
$$

An intuitive picture which displays why this sequence of EFTs is relevant is shown in Fig. 2. We are interested in events where the top quarks are produced close to their mass shell as characterized by the condition in Eq. (1). At the production scale $Q$, the invariant mass of the top and antitop quarks can still fluctuate with $\hat{s}_{t, \bar{t}} \sim Q$ due to its interactions with hard gluons of characteristic momentum $p_{h} \sim Q$. In the first step, when switching to SCET, these hard modes are integrated out and we expand in $m / Q \ll 1$. SCET makes it simple to separate the physics associated with (i) the top jet, (ii) the antitop jet, and (iii) the soft cross talk between the jets. After the implementation of this factorization theorem, each top jet and the soft cross talk can be studied independently in the field theory. The factorization theorem tells us how to tie them together. Now in SCET the invariant mass of the top quark fluctuates with $\hat{s}_{t, \bar{t}} \sim m$, so we still have to remove these large momentum fluctuations to describe the desired kinematic 
region where $\Gamma \sim \hat{s}_{t, \bar{t}} \ll m$. Such invariant mass fluctuations are analogous to those encountered in HQET for a bottom quark inside a $B$ meson

$$
(m v+k)^{2}-m^{2}=2 m v \cdot k+k^{2} \sim 2 m \Lambda_{\mathrm{QCD}},
$$

with the difference that for the unstable top quark $v \cdot k \rightarrow$ $v \cdot k+i \Gamma / 2$. Since top quarks decay before they have a chance to hadronize, the top width $\Gamma$ adopts the role $\Lambda_{\mathrm{QCD}}$ plays for the $B$ meson. Keeping in mind that the tops are highly boosted and unstable, we actually match onto two boosted versions of HQET, one for the top and one for the antitop. A discussion of the necessary SCET and HQET theoretical ingredients is given in the following subsections.

\section{A. SCET with masses}

SCET is an effective theory describing the interactions of soft and collinear particles, which are characterized by the scaling of their momenta. In this framework it is convenient to introduce the four-vectors

$$
n^{\mu}=(1, \vec{n}), \quad \bar{n}^{\mu}=(1,-\vec{n}),
$$

where $\vec{n}$ can be thought of as the direction of the top jet and $-\vec{n}$ as the direction of the antitop jet $\left(\vec{n}^{2}=1, n^{2}=0, \vec{n}^{2}=\right.$ 0 ). Any momentum can then be decomposed as

$$
p^{\mu}=n \cdot p \frac{\bar{n}^{\mu}}{2}+\bar{n} \cdot p \frac{n^{\mu}}{2}+p_{\perp}^{\mu},
$$

and we denote momentum components in this light-cone basis as $\left(p^{+}, p^{-}, p_{\perp}\right)=\left(n \cdot p, \bar{n} \cdot p, p_{\perp}\right)$. The square of the momentum vector $p^{\mu}$ then reads $p^{2}=p^{+} p^{-}+p_{\perp}^{2}$. It is also convenient to denote the momentum of collinear particles in the $\vec{n}$ and $-\vec{n}$ directions by the subscripts $n$ and $\bar{n}$, respectively, which corresponds to the large energy modes in the corresponding jets. Thus we have collinear labels

$n$ for the top jet, $\quad \bar{n}$ for the antitop jet.

The momentum of soft particles that communicate between the jets will be denoted by a subscript $s$. We also have mass modes that are required in order to describe certain top quark vacuum polarization loops. The momenta of the collinear, mass, and soft modes ${ }^{2}$ have the typical scalings shown in Table I in the SCET column, where $\lambda$ is the small expansion parameter. A particle with components scaling as $\left(\lambda^{2}, 1, \lambda\right)$ has a small $\perp$-momentum relative to

\footnotetext{
${ }^{2}$ In some factorization theorems it is necessary to distinguish between soft and ultrasoft particles, and between two versions of SCET: called SCET $\mathrm{I}_{\mathrm{I}}$ and $\mathrm{SCET}_{\mathrm{II}}$. In this paper we only deal with SCET $_{\text {I }}$ with ultrasoft gluons. For simplicity we will therefore simply use the term soft modes. For modes with momenta $p^{\mu} \sim$ $(m, m, m)$ that are specific to the massive SCET theory, we use the term "mass modes."
}

its energy and is said to be collinear to the $n^{\mu}$ direction etc. Both $\lambda$ and the hard scale $Q$ have a size that depends on the particular process under study. For example, in $B \rightarrow X_{s} \gamma$ the hard scale is the $b$-quark mass $m_{b}$, and the expansion parameter is $\sqrt{\Lambda_{\mathrm{QCD}} / m_{b}}$. For pair production of top jets, the hard scale $Q$ is the center of mass energy, and the SCET expansion parameter is

$$
\lambda \sim \frac{m}{Q}
$$

It follows that the typical virtuality of the collinear, mass, and soft modes in SCET satisfy

$$
p_{n}^{2} \sim p_{\bar{n}}^{2} \sim m^{2}, \quad p_{m}^{2} \sim m^{2}, \quad \text { and } \quad p_{s}^{2} \sim \frac{m^{4}}{Q^{2}} .
$$

Since $m^{4} / Q^{2} \gg \Lambda_{\mathrm{OCD}}^{2}$, the soft modes in this theory still contain perturbative components as well as the underlying nonperturbative dynamics at smaller scales. Using $m=$ $171 \mathrm{GeV}$ this is true for $Q \lesssim 40 \mathrm{TeV}$ i.e. for any conceivable c.m. energy of a future linear collider. The soft particles correspond to modes with wavelengths that allow cross talk between the two jets. In addition, at two-loop order the soft gluons in SCET interact with virtual top quarks which are described by the mass modes indicated in Table I. These mass modes do not interact directly with the collinear fields and only appear as virtual effects for our observable, because we only consider cases where $s_{t, \bar{t}} \ll$ Qm. [As discussed below Eq. (23).] In addition we have virtual collinear top quarks that can interact with the collinear particles through the collinear Lagrangian. The $n$-collinear, $\bar{n}$-collinear, mass modes, and soft modes are described by separate quark and gluon fields which are also listed in Table I. Hard modes involving momenta $p^{\mu} \sim Q$ have already been integrated out when QCD is matched onto SCET.

At leading order the SCET Lagrangian for collinear particles in different directions can be written as a soft Lagrangian plus a sum of collinear terms [41], $\mathcal{L}^{(0)}=$ $\mathcal{L}_{s}+\sum_{n_{i}} \mathcal{L}_{n_{i}}^{(0)}$. The sum satisfies the constraint $n_{i} \cdot n_{j} \gg$ $\lambda^{2}$ for $i \neq j$, with the choice of $\lambda$ determining what is meant by distinct collinear directions. The collinear particles in different sectors only interact via soft gluon exchange or interactions in external operators. When the $\perp$-momentum of the collinear particles is of the same size as the quark mass the result for the leading order collinear Lagrangian [26,27] must include the quark mass terms derived in Ref. [29] (see also Ref. [50]). The collinear quark Lagrangian for the direction $n$ is therefore given by 
TABLE I. Summary of the fields required in SCET and bHQET. The first field in each bracket is a quark and the second is a gluon. The scaling of momentum components is given for $\left(p^{+}, p^{-}, p^{\perp}\right)$. After factorization, the soft fields on the last line generate a cross talk theory that communicates with collinear fields in both SCET and bHQET through two kinematic variables. $\Delta$ is the scale for the soft modes.

\begin{tabular}{|c|c|c|c|}
\hline \multicolumn{2}{|c|}{$\operatorname{SCET}[\lambda \sim m / Q \ll 1]$} & \multicolumn{2}{|c|}{ bHQET $[\Gamma / m \ll 1]$} \\
\hline$n$-collinear $\left(\xi_{n}, A_{n}^{\mu}\right)$ & $p_{n}^{\mu} \sim Q\left(\lambda^{2}, 1, \lambda\right)$ & $n$-ucollinear $\left(h_{v_{+}}, A_{+}^{\mu}\right)$ & $k^{\mu} \sim \Gamma\left(\lambda, \lambda^{-1}, 1\right)$ \\
\hline $\begin{array}{c}\bar{n} \text {-collinear }\left(\xi_{\bar{n}}, A_{\bar{n}}^{\mu}\right) \\
\text { mass modes }\left(q_{m}, A_{m}^{\mu}\right)\end{array}$ & $\begin{aligned} p_{\bar{n}}^{\mu} & \sim Q\left(1, \lambda^{2}, \lambda\right) \\
p_{m}^{\mu} & \sim Q(\lambda, \lambda, \lambda)\end{aligned}$ & $\bar{n}$-ucollinear $\left(h_{v_{-}}, A_{-}^{\mu}\right)$ & $k^{\mu} \sim \Gamma\left(\lambda^{-1}, \lambda, 1\right)$ \\
\hline Cross talk: soft $\left(q_{s}, A_{s}^{\mu}\right)$ & $p_{s}^{\mu} \sim Q\left(\lambda^{2}, \lambda^{2}, \lambda^{2}\right)$ & same soft $\left(q_{s}, A_{s}^{\mu}\right)$ & $p_{s}^{\mu} \sim(\Delta, \Delta, \Delta)$ \\
\hline
\end{tabular}

$$
\begin{aligned}
\mathcal{L}_{q n}^{(0)}= & \bar{\xi}_{n}\left[i n \cdot D_{s}+g n \cdot A_{n}+\left(i \not D_{c}^{\perp}-m\right) W_{n} \frac{1}{\bar{n} \cdot \mathcal{P}}\right. \\
& \left.\times W_{n}^{\dagger}\left(i \not D_{c}^{\perp}+m\right)\right] \frac{\bar{h}}{2} \xi_{n},
\end{aligned}
$$

with $D_{c}^{\perp} \sim m \gg D_{s}^{\perp}$. There is also an $n$-collinear Lagrangian for gluons [28]. Here the soft and collinear covariant derivatives are

$$
i D_{s}^{\mu}=i \partial^{\mu}+g A_{s}^{\mu}, \quad i D_{c}^{\mu}=\mathcal{P}^{\mu}+g A_{n}^{\mu},
$$

where $\mathcal{P}^{\mu}$ is a label operator picking out the large collinear momentum of order $Q$ and $Q \lambda$ of a collinear field [27], while the partial derivative acts on the residual momentum components $\partial^{\mu} \sim \lambda^{2}$. The term $W_{n}$ is the momentum space Wilson line built out of collinear gluon fields

$$
W_{n}(x)=\sum_{\text {perms }} \exp \left(-\frac{g}{\overline{\mathcal{P}}} \bar{n} \cdot A_{n}(x)\right)
$$

We also note that Eq. (13) is the bare Lagrangian. In particular, any mass definition can be chosen for $m$ through an appropriate renormalization condition without breaking the power counting. At $\mathcal{O}\left(\alpha_{s}\right)$ these mass schemes are the same as those in QCD [51], because the self-energy graphs are directly related.

An example of an external operator that connects different collinear sectors is the jet production current, which couples to the $\gamma^{*}$ or $Z^{*}$. In QCD the production matrix element is $\left\langle X\left|\mathcal{J}_{a, v}^{\mu}\right| 0\right\rangle$ where $\langle X|$ is the final state. The required vector and axial currents are given by

$$
\mathcal{J}_{v}^{\mu}(x)=\bar{\psi}(x) \gamma^{\mu} \psi(x), \quad \mathcal{J}_{a}^{\mu}(x)=\bar{\psi}(x) \gamma^{\mu} \gamma_{5} \psi(x),
$$

and for convenience we will adopt the shorthand notation $\mathcal{J}_{i}^{\mu}=\bar{\psi}(x) \Gamma_{i}^{\mu} \psi(x)$. The matching relation of these QCD currents to SCET currents is given by the convolution formula [26]

$$
\mathcal{J}_{i}^{\mu}(0)=\int d \omega d \bar{\omega} C(\omega, \bar{\omega}, \mu) J_{i}^{(0) \mu}(\omega, \bar{\omega}, \mu),
$$

where $C$ contains short-distance dynamics at the scale $Q$, while $J_{i}^{(0) \mu}$ describes fluctuations at all longer distance scales. In the presence of multiple collinear fields, as well as modes scaling like our mass modes and soft modes, the construction of currents in SCET has been discussed in great detail in Ref. [41]. Interactions between the mass modes and the collinear modes produce off-shell particles, which when integrated out leave residual interactions through Wilson lines in the SCET current. The SCET production current at leading order in $\lambda$ is given by

$$
J_{i}^{(0) \mu}(\omega, \bar{\omega}, \mu)=\bar{\chi}_{n, \omega}(0) S_{n}^{\dagger} \Gamma_{i}^{\mu} S_{\bar{n}} \chi_{\bar{n}, \bar{\omega}}(0),
$$

where $\chi_{n, \omega}(0)=\delta(\omega-\bar{n} \cdot \mathcal{P})\left(W_{n}^{\dagger} \xi_{n}\right)(0)$ and $\chi_{\bar{n}, \bar{\omega}}(0)=$ $\delta(\bar{\omega}-n \cdot \mathcal{P})\left(W_{\bar{n}}^{\dagger} \xi_{\bar{n}}\right)(0)$. The mass mode Wilson lines $S_{n}^{\dagger}$ and $S_{\bar{n}}$ will be described below. Here the (0) indicates that the fields are at coordinate $x^{\mu}=0$, and we recall that this $x^{\mu}$ dependence carries information about the residual momenta at the scale $Q \lambda^{2}=m^{2} / Q$. The dependence on larger momenta is encoded in labels on the collinear fields [27], and, for example, $\delta(\omega-\bar{n} \cdot P)$ forces the total minus-label-momentum of $\left(W_{n}^{\dagger} \xi_{n}\right)$ to be $\omega$. We also use the notation $\chi_{n}=\left(W_{n}^{\dagger} \xi_{n}\right)$ and $\chi_{\bar{n}}=\left(W_{\bar{n}}^{\dagger} \xi_{\bar{n}}\right)$.

One can decouple the soft and collinear modes in $\mathcal{L}_{q n}^{(0)}$ by performing a field redefinition on collinear fields [28]

$$
\xi_{n} \rightarrow Y_{n} \xi_{n}, \quad A_{n}^{\mu} \rightarrow Y_{n} A_{n}^{\mu} Y_{n}^{\dagger},
$$

where $Y_{n}$ is a soft Wilson line

$$
Y_{n}(x)=\overline{\mathrm{P}} \exp \left(-i g \int_{0}^{\infty} d s n \cdot A_{s}(n s+x)\right) .
$$

This gives

$$
Y_{n}^{\dagger}(x)=\mathrm{P} \exp \left(i g \int_{0}^{\infty} d s n \cdot A_{s}(n s+x)\right),
$$

which satisfies $Y_{n}^{\dagger} Y_{n}=1$. For two-jet production the factorization is most transparent [34] with the reference point $s_{0}=\infty$ shown in Eq. (20). The gluon fields are either antipath-ordered (for $\overline{\mathrm{P}}$ ) or path-ordered (for P). We use the same Wilson line for both the quark and antiquark parts of $\xi_{n}$. Another possibility is to make different field redefinitions on the particle and antiparticle parts of the fields [52]. In fact, all results are independent of the choice of reference point in the field redefinition; the path is determined entirely by changes the field redefinition induces on the operators and the interpolating fields for the states [53]. 
The mass mode Wilson line $S_{n}(x)$ is defined in an identical manner to Eq. (20), but with $n \cdot A_{s} \rightarrow n \cdot A_{m}$. In order to avoid double counting with the effects contained in the soft Wilson lines the mass mode $A_{m}^{\mu}$ fields are defined with zero-bin subtractions for the soft region [54], and we have mass mode top quarks $\psi_{m}$ with a mass $m$. Any graphs with mass mode gluons that do not involve a top-bubble from $\psi_{m}$ fields are exactly canceled by these zero-bin subtractions. Thus the mass modes only contribute in these vacuum polarization graphs. The soft gluons can also couple to the $\psi_{m}$ fields; however, they do so with a multipole expansion and therefore do not inject momentum into the closed $\psi_{m}$ loop.

After the change of variable in Eq. (19) the leading order SCET collinear quark Lagrangian and current become

$$
\begin{aligned}
\mathcal{L}_{n q}^{(0)}= & \bar{\xi}_{n}\left[i n \cdot \partial+g n \cdot A_{n}\right. \\
& \left.+\left(i \not D_{c}^{\perp}-m\right) W_{n} \frac{1}{\overline{\mathcal{P}}} W_{n}^{\dagger}\left(i \not D_{c}^{\perp}+m\right)\right] \frac{\bar{h}}{2} \xi_{n}, \\
J_{i}^{(0) \mu}= & \bar{\chi}_{n, \omega} Y_{n}^{\dagger} S_{n}^{\dagger} \Gamma_{i}^{\mu} S_{\bar{n}} Y_{\bar{n}} \chi_{\bar{n}, \bar{\omega}}(0),
\end{aligned}
$$

where we used the property $Y_{n}^{\dagger} n \cdot D_{s} Y_{n}=n \cdot \partial$. The only coupling of the soft gluon to the collinear quark was through in $\cdot D_{s}$ which is no longer present (and a similar property occurs in the collinear gluon action). These soft couplings reappear as Wilson lines in the current as shown above. Hence we have achieved soft collinear decoupling in the Lagrangian and the current.

In the two-jet process we wish to factorize we must also consider the transformation property of the state $|X\rangle$ under Eq. (19). For manipulations in the factorization theorem for two-jet production we decompose the state into collinear and soft pieces,

$$
\langle X|=\left\langle X_{n} X_{\bar{n}} X_{s}\right| .
$$

Note that this decomposition is only valid for the states we are interested in for describing the dijet region, not for a general state in QCD. Since there is always at least one $n$-collinear and one $\bar{n}$-collinear particle, we do not consider any mass modes, $X_{m}$, in these states either. The presence of a mass mode would induce an invariant mass $p_{X}^{2}=\left(p_{n}+\right.$ $\left.p_{m}\right)^{2} \simeq p_{n}^{-} p_{m}^{+} \simeq Q m \gg m^{2}$, which would make it impossible to satisfy the invariant mass condition required to study the peak region. Therefore the mass modes will only appear as virtual contributions. The collinear states $\left\langle X_{n}\right|$ and $\left\langle X_{\bar{n}}\right|$ are a color triplet and color antitriplet, just like a quark and antiquark state. Therefore, we must consider how these collinear states transform under the change in the action induced by Eq. (19). However, because these color triplet states can be derived from the out states at large time, $t \rightarrow \infty$, they are not affected by the field redefinition with reference point at $\infty$ [53]. With the current at $x$, we therefore have

$$
\left\langle X\left|\mathrm{~T}\left\{\bar{\chi}_{n, \omega} S_{n}^{\dagger} \Gamma S_{\bar{n}} \chi_{\bar{n}, \bar{\omega}}\right\}\right| 0\right\rangle \rightarrow\left\langle X\left|\mathrm{~T}\left\{\bar{\chi}_{n, \omega} Y_{n}^{\dagger} S_{n}^{\dagger} \Gamma S_{\bar{n}} Y_{\bar{n}} \chi_{\bar{n}, \bar{\omega}}\right\}\right| 0\right\rangle .
$$

Here the T reminds us to keep the proper time-ordering of the $A^{a}(x)$ gluon fields in the $Y$ 's. There is no ordering issue between fields in $Y_{\bar{n}}$ with those in $Y_{n}^{\dagger}$, since they are spacelike separated and commute [34]. We also need the complex conjugate of Eq. (24) for the matrix element, which is

$$
\left\langle 0\left|\mathrm{~T}\left\{\bar{\chi}_{\bar{n}, \bar{\omega}} S_{\bar{n}}^{\dagger} \Gamma S_{n} \chi_{n, \omega}\right\}\right| X\right\rangle \rightarrow\left\langle 0\left|\overline{\mathrm{T}}\left\{\bar{\chi}_{\bar{n}, \bar{\omega}} Y_{\bar{n}}^{\dagger} S_{\bar{n}}^{\dagger} \bar{\Gamma} S_{n} Y_{n} \chi_{n, \omega}\right\}\right| X\right\rangle,
$$

where $\overline{\mathrm{T}}$ is antitime-ordering. Note that

$$
\begin{aligned}
& \mathrm{T}\left(Y_{\bar{n}}\right)^{T}=\left(Y_{\bar{n}}^{\dagger}\right)^{*}=\bar{Y}_{\bar{n}}^{\dagger}=\mathrm{P} \exp \left(i g \int_{0}^{\infty} d s \bar{n} \cdot \bar{A}_{s}(\bar{n} s+x)\right), \\
& \overline{\mathrm{T}}\left(Y_{\bar{n}}^{\dagger}\right)^{T}=\left(Y_{\bar{n}}\right)^{*}=\bar{Y}_{\bar{n}}=\overline{\mathrm{P}} \exp \left(-i g \int_{0}^{\infty} d s \bar{n} \cdot \bar{A}_{s}(\bar{n} s+x)\right),
\end{aligned}
$$

where $\bar{A}_{s}=A_{s}^{A} \bar{T}^{A}$, with $\bar{T}^{A}=-\left(T^{A}\right)^{T}$ the generator for the $\overline{3}$ representation, and the superscript $T$ is the transpose with respect to the color indices of the fundamental representation. If we switch to these barred Wilson lines then the time-ordering and antitime-ordering becomes redundant. Equation (26) applies equally well for the $S$ Wilson lines. Considering the squared matrix element for the cross section we find

$$
\begin{aligned}
& \left\langle 0\left|\overline{\mathrm{T}}\left\{\bar{\chi}_{\bar{n}, \bar{\omega}^{\prime}} Y_{\bar{n}}^{\dagger} S_{\bar{n}}^{\dagger} \bar{\Gamma} S_{n} Y_{n} \chi_{n, \omega^{\prime}}\right\}\right| X\right\rangle\left\langle X\left|\mathrm{~T}\left\{\bar{\chi}_{n, \omega} Y_{n}^{\dagger} S_{n}^{\dagger} \Gamma S_{\bar{n}} Y_{\bar{n}} \chi_{\bar{n}, \bar{\omega}}\right\}\right| 0\right\rangle \\
& \quad=\left\langle 0\left|\bar{\chi}_{\bar{n}, \bar{\omega}^{\prime}}^{a}\left(\bar{Y}_{\bar{n}}\right)^{b a}\left(\bar{S}_{\bar{n}}\right)^{b^{\prime} b}\left(\bar{\Gamma} S_{n} Y_{n} \chi_{n, \omega^{\prime}}\right)^{b^{\prime}}\right| X\right\rangle\left\langle X\left|\left(\bar{\chi}_{n, \omega} Y_{n}^{\dagger} S_{n}^{\dagger} \Gamma\right)^{c^{\prime}}\left(\bar{S}_{\bar{n}}^{\dagger}\right)^{c c^{\prime}}\left(\bar{Y}_{\bar{n}}^{\dagger}\right)^{d c} \chi_{\bar{n}, \bar{\omega}}^{d}\right| 0\right\rangle \\
& \quad=\mathcal{M}(m, \mu)\left\langle 0\left|\bar{\chi}_{\bar{n}, \bar{\omega}^{\prime}}^{a}\left(\bar{Y}_{\bar{n}}\right)^{b a}\left(\bar{\Gamma} Y_{n} \chi_{n, \omega^{\prime}}\right)^{b^{\prime}}\right| X\right\rangle\left\langle X\left|\left(\bar{\chi}_{n, \omega} Y_{n}^{\dagger} \Gamma\right)^{c^{\prime}}\left(\bar{Y}_{\bar{n}}^{\dagger}\right)^{d c} \chi_{\bar{n}, \bar{\omega}}^{d}\right| 0\right\rangle,
\end{aligned}
$$

where $a, b, c, d, b^{\prime}, c^{\prime}$ are color indices. The decoupling of soft gluons in Eq. (27) is identical to that in massless twojet production, and ignoring the mass mode Wilson lines the discussion above agrees with the SCET derivation in Ref. [34], as well as the original derivation in Refs. [55,56].
To obtain the last line in Eq. (27) we note that the Dirac structures $\Gamma$ and $\bar{\Gamma}$ are color singlets, and that the mass mode Wilson lines can be separated into vacuum matrix elements since there are no mass modes in the states. Furthermore 


$$
\left\langle 0\left|\left(\bar{S}_{\bar{n}}\right)^{b^{\prime} b}\left(S_{n}\right)^{b^{\prime} a}\right| 0\right\rangle=\frac{\delta^{b a}}{N_{c}}\left\langle 0\left|\left(\bar{S}_{\bar{n}}\right)^{b^{\prime} a^{\prime}}\left(S_{n}\right)^{b^{\prime} a^{\prime}}\right| 0\right\rangle,
$$

with an analogous result for $\left\langle 0\left|\left(S_{n}^{\dagger}\right)^{a c^{\prime}}\left(\bar{S}_{\bar{n}}^{\dagger}\right)^{c c^{\prime}}\right| 0\right\rangle$, so this contracts the color indices on either side of the product of soft Wilson-line factors. Thus defining

$$
\mathcal{M}(m, \mu) \equiv \frac{1}{N_{c}^{2}}\left|\left\langle 0\left|\bar{S}_{\bar{n}}^{a b} S_{n}^{a b}\right| 0\right\rangle\right|^{2},
$$

we are left with the matrix element shown on the last line of Eq. (27). Here $\mathcal{M}(m, \mu)=1+\mathcal{O}\left(\alpha_{s}^{2}\right)$.

The soft collinear decoupling property is crucial to organizing the physics of the massive two-jet problem. As we will show in Sec. III, there is a factorization theorem in SCET that decouples the soft and collinear modes at leading order which allows us to study the physics of each jet independently. The cross talk is confined to a simple top-mass independent vacuum matrix element involving the $Y$ Wilson lines,

$$
\left\langle 0\left|\left(\bar{Y}_{\bar{n}}\right)^{b a}\left(Y_{n}\right)^{b c}\right| X_{s}\right\rangle\left\langle X_{s}\left|\left(Y_{n}^{\dagger}\right)^{c d}\left(\bar{Y}_{\bar{n}}^{\dagger}\right)^{a d}\right| 0\right\rangle,
$$

which agrees with the corresponding soft matrix element for massless quark production [36,56,57] and which will eventually determine the soft function $S_{\text {hemi }}\left(\ell^{+}, \ell^{-}\right)$to be used in Eq. (3). As we also show in Sec. III, the precise definition of the soft function $S$ depends on the prescription that is used for how the momenta of soft particles enter the top and antitop invariant masses $\hat{s}_{t}$ and $\hat{s}_{\bar{t}}$, respectively. In the next subsection we describe how the matrix element in Eq. (30) is modified when we integrate out the top quark mass.

Finally, in SCET because the top quark mass $m$, and mass of the $W$ boson, $m_{W}$, are still low energy scales, the decay of an $n$-collinear top quark is simply described by the full electroweak interaction,

$$
\mathcal{L}_{\mathrm{ew}}=\frac{g_{2}}{\sqrt{2}} \bar{b} W_{\mu}^{-} \gamma^{\mu} P_{L} t+\frac{g_{2}}{\sqrt{2}} \bar{t} W_{\mu}^{+} \gamma^{\mu} P_{L} b,
$$

where $G_{F}=\sqrt{2} g_{2}^{2} /\left(8 m_{W}^{2}\right)$ is the Fermi constant. This treatment is consistent since we can treat the top decay as fully inclusive up to $\mathcal{O}\left(\mathrm{m}^{2} / \mathrm{Q}^{2}\right)$.

The collinear $A_{n}$ and $A_{\bar{n}}$ gluons in SCET can induce fluctuations $\hat{s}_{t}, \hat{s}_{\bar{t}} \sim m$. Once we restrict ourselves to events with $\Gamma \sim \hat{s}_{t}, \hat{s}_{\bar{t}} \ll m$, i.e. we force the top quark and antiquark to remain close to their mass shell, the situation looks very much like two distinct copies of HQET in boosted frames. There was nothing special in the dynamics that sets the scale $m^{2} / Q$ for the soft interactions, and so we call $\Delta$ the scale that controls the soft cross talk. In the field theory $\Delta$ will be defined as the scale where we model or fit the primordial soft function. Generally we will take $m \gg$ $\Delta \sim \Gamma$, although any value $\Delta>\Lambda_{\mathrm{QCD}}$ can be considered. So we must switch from SCET onto these HQET theories, and also consider what happens to the decay interaction in Eq. (31). We describe the boosted HQET theories in detail in the next section, and we also discuss how the soft cross talk interactions remain active when the fluctuations at the top-mass scale $m$ are integrated out.

Since the above Lagrangians and currents are leading order in $\lambda$, it is natural to ask about the role of power corrections. As it turns out, higher order Lagrangians and currents give corrections to our analysis at $\mathcal{O}\left(\alpha_{s} m / Q\right)$, $\mathcal{O}(\Delta / Q), \quad \mathcal{O}\left(m^{2} / Q^{2}\right), \quad$ or $\mathcal{O}(\Gamma / m)$. The absence of $\mathcal{O}(m / Q)$ implies that the $m / Q$ expansion does not significantly modify the top-mass determination. The leading action contains all $m / Q$ corrections that do not involve an additional perturbative gluon, so the corrections are $\mathcal{O}\left(\alpha_{s} m / Q\right)$. We have also verified that at tree level the $m / Q$ corrections to the SCET current [50] vanish when contracted with the leptonic tensor. Furthermore, many of the higher order $m / Q$ corrections have the form of normalization corrections, and thus do not change the shape of the invariant mass distribution. Subleading soft interactions are $\mathcal{O}(\Delta / Q)$. The interplay of our hemisphere invariant mass variable with the top decay can induce $\mathcal{O}\left(\mathrm{m}^{2} / Q^{2}\right)$ corrections, as we discuss later on. Finally there will be power corrections of $\mathcal{O}(\Gamma / m)$ in bHQET.

\section{B. Boosted HQET with unstable particles and soft cross talk}

Boosted heavy quarks. - HQET [42-46] is an effective theory describing the interactions of a heavy quark with soft degrees of freedom, and also plays a crucial role for jets initiated by massive unstable particles in the peak regions close to the heavy particles mass shell. The momentum of a heavy quark interacting with soft degrees of freedom can be written as

$$
p^{\mu}=m v^{\mu}+k^{\mu},
$$

where $k^{\mu}$ denotes momentum fluctuations due to interactions with the soft degrees of freedom and is much smaller than the heavy quark mass $\left|k^{\mu}\right| \ll m$. Also typically $v^{\mu} \sim$ 1 so that we are parametrically close to the top quark rest frame, $v^{\mu}=(1, \overrightarrow{0})$.

In the top quark rest frame we have $\Gamma \lesssim k^{\mu} \ll m$, where $k^{\mu}$ refers to momentum fluctuations of the top due to interactions with gluons collinear to its direction, which preserve the invariant mass conditions $\Gamma \sim \hat{s}_{t}, \hat{s}_{\bar{t}} \ll m$. For our top quark analysis, the center of mass frame is the most convenient to setup the degrees of freedom. In this frame the gluons collinear to the top quark which preserve the invariant mass condition will be called ultracollinear (ucollinear) in the $n$ direction. A different set of $\bar{n}$-ucollinear gluons interact with the antitop quark which moves in the $\bar{n}$ direction. The leading order Lagrangian of the EFT describing the evolution and decay of the top or antitop close to its mass shell is given by 


$$
\begin{aligned}
& \mathcal{L}_{+}=\bar{h}_{v_{+}}\left(i v_{+} \cdot D_{+}-\delta m+\frac{i}{2} \Gamma\right) h_{v_{+}}, \\
& \mathcal{L}_{-}=\bar{h}_{v_{-}}\left(i v_{-} \cdot D_{-}-\delta m+\frac{i}{2} \Gamma\right) h_{v_{-}},
\end{aligned}
$$

where the + and - subscripts refer to the top and antitop sectors, respectively, and $i D_{ \pm}^{\mu}=i \partial^{\mu}+g A_{ \pm}^{\mu}$. These HQETs represent an expansion in $\Gamma / m$. The HQET field $h_{v_{+}}$annihilates top quarks, while $h_{v_{-}}$creates antitop quarks. In the c.m. frame the components of $k^{\mu}$ are no longer homogeneous in size, and $v_{ \pm}^{\mu} \mathcal{f}$. Instead for the $(+,-, \perp)$ components we have

$$
\begin{array}{lll}
v_{+}^{\mu}=\left(\frac{m}{Q}, \frac{Q}{m}, \mathbf{0}_{\perp}\right), & k_{+}^{\mu} \sim \Gamma\left(\frac{m}{Q}, \frac{Q}{m}, 1\right), \\
\boldsymbol{v}_{-}^{\mu}=\left(\frac{Q}{m}, \frac{m}{Q}, \mathbf{0}_{\perp}\right), & k_{-}^{\mu} \sim \Gamma\left(\frac{Q}{m}, \frac{m}{Q}, 1\right) .
\end{array}
$$

Note that the $\Gamma$ in Eq. (34) can be replaced by a larger scale, of order $\hat{s}$, as long as this scale is much less than $m$. Equation (34) is easily obtained by boosting from the rest frame of the top and antitop, respectively, with a boost factor of $Q / \mathrm{m}$. In this naming scheme we will continue to call the gluons that govern the cross talk between top and antitop jets soft. We emphasize that they are not included in $\mathcal{L}_{ \pm}$, since they have nothing to do with the gluons in standard HQET. Soft gluon interactions will be added below. To avoid double counting between the soft gluons, the ultracollinear gluons are defined with zero-bin subtractions [54], so that for example $\bar{n} \cdot k_{+} \neq 0$ and $n \cdot k_{-} \neq 0$. Finally, since HQET is applied for $\mu<m$ there are no analogs of the SCET mass modes in this theory. All effects associated with virtual top quark loops are integrated out at the scale $m$.

The leading order Lagrangians $\mathcal{L}_{ \pm}$contain a residual mass term $\delta m$ which has to be chosen according to the desired top quark mass scheme. For a given top mass scheme $m$, the residual mass term is determined by its relation to the pole mass $m_{\text {pole }}=m+\delta m$. Anticipating that we have to switch to a properly defined short-distance mass definition [58-61] when higher order QCD corrections are included, we note that only short-distance mass definitions are allowed which do not violate the power counting of the bHQET theories, $\delta m \sim \Gamma$. This excludes, for example, the use of the well-known $\overline{\mathrm{MS}}$ mass, since in this scheme $\delta m \sim \alpha_{s} m \gg \Gamma$. In practice, this means that using the $\overline{\mathrm{MS}}$ mass leads to an inconsistent perturbative expansion as explained in Sec. III G. This is the reason why the $\overline{\text { MS }}$ mass cannot be measured directly from reconstruction.

The leading order Lagrangians $\mathcal{L}_{ \pm}$also contain top width terms $i \Gamma / 2$. An effective field theory treatment of the evolution and decay of a massive unstable particle close to its mass shell was developed in [12,19,31,47-49]. The examples treated in these references were the resonant production of a single unstable scalar particle, and the leading and subleading width corrections to threshold $t \bar{t}$ production. In our case, we deal with the energetic pair production of massive unstable fermions, and we arrive at two copies of this unstable HQET corresponding to the top and antitop sectors. In these two HQET theories we treat the top and antitop decays as totally inclusive, since we do not require detailed differential information on the decay products. So the total top width $\Gamma$ appears as an imaginary mass term in $\mathcal{L}_{ \pm}$, which is obtained by simply matching the imaginary part of the top and antitop self-energy graphs from SCET onto bHQET. As we show in Sec. III, this inclusive treatment of the top decay is consistent with the hemisphere invariant mass definition we employ in this work up to power corrections of order $(m / Q)^{2}$. We will come back to the role of higher order power corrections in the treatment of the finite top lifetime at the end of this section.

Soft interactions. - Let us consider how the soft gluons interact with our heavy quarks in each bHQET. For a heavy quark in the boosted frame we consider interactions with soft gluons of momentum

$$
\ell^{\mu} \lesssim(\Delta, \Delta, \Delta) .
$$

Our main interest is in the case $\Delta \lesssim \Gamma$, but it is useful to keep a more general $\Delta \geq \Lambda_{\mathrm{QCD}}$ for the moment. We wish to demonstrate that these gluons are still entirely described by the cross talk matrix element in Eq. (30), and that this is true without needing to expand in the ratio of $\Delta$ to $\Gamma$. Or in other words, that the simple eikonal propagators for the soft gluon attachments to the energetic tops remain valid even below the mass of the quarks and even in the presence of the top width. Our demonstration assumes the reader is quite familiar with Ref. [28]. To prove this we go back to the original SCET Lagrangian in Eq. (13) prior to the field redefinition and match the soft interactions onto the HQET theory. This gives the same Lagrangian as in Eq. (33) but with replacements

$$
\begin{aligned}
& i D_{+}^{\mu} \rightarrow i \mathcal{D}_{+}^{\mu}=i \tilde{\partial}_{+}^{\mu}+g A_{+}^{\mu}+\frac{\bar{n}^{\mu}}{2} g n \cdot A_{s}, \\
& i D_{-}^{\mu} \rightarrow i \mathcal{D}_{-}^{\mu}=i \tilde{\partial}_{-}^{\mu}+g A_{-}^{\mu}+\frac{n^{\mu}}{2} g \bar{n} \cdot A_{s} .
\end{aligned}
$$

The new covariant derivatives $\mathcal{D}_{ \pm}$also appear in the pure gluon action responsible for the ultracollinear gluon kinetic term. The nature of the expansion for different momenta in $i \tilde{\partial}^{\mu}$ will depend on the size of the soft scale $\Delta$ relative to the smallest ultracollinear components $m \Gamma / Q$ displayed in Eq. (34). Note in this comparison that the width is suppressed by a factor of $m / Q$. Physically this factor is easy to understand, it is simply the time-dilation of the width of the energetic top quark from the point of view of the soft gluons. The boost factor is encoded in $v_{+} \cdot \bar{n}=v_{-} \cdot n=$ $Q / m$.

For our analysis we also need the effective current in the bHQET theories that corresponds to the SCET current in 
Eq. (18). It is

$$
J_{\text {bHQET }}^{\mu}=\left(\bar{h}_{v_{+}} W_{n}\right) \Gamma_{i}^{\mu}\left(W_{\bar{n}}^{\dagger} h_{v_{-}}\right),
$$

where the Wilson lines are the same as $W_{n}$ and $W_{\bar{n}}^{\dagger}$ in SCET, except here we have gluons $\bar{n} \cdot A_{+}$with path along $\bar{n}^{\mu}$ for $W_{n}$, and $n \cdot A_{-}$with path along $n^{\mu}$ for $W_{\bar{n}}^{\dagger}$. The simplest way to derive this result is to note that the two collinear sectors in the SCET current in Eq. (18) do not directly interact, and neither do the two sectors of the two bHQETs. In the rest frame of the top quark, for example, the matching is simply $\left(W_{n}^{\dagger} \psi_{s}\right) \rightarrow\left(W_{n}^{\dagger} h_{v_{+}}\right)$, where $\psi_{s}$ is a field for the top quark near its rest frame. Boosting this result gives the matching for the top quark field in Eq. (37), and the result for the antitop quark is analogous. The dynamics of the $B_{+}$and $B_{-}$jet functions will be defined by the two interpolating field operators $\left(W_{n}^{\dagger} h_{v_{+}}\right)$and $\left(W_{\bar{n}}^{\dagger} h_{v_{-}}\right)$and is governed by the Lagrangians $\mathcal{L}_{+}$and $\mathcal{L}_{-}$, respectively.

Let us now come back to the derivation of Eq. (30) from bHQET. For convenience we start by taking both scales the same size, $m \Gamma / Q \sim \Delta$. (Below we will show that the same result is obtained for the case where $m \Gamma / Q \ll \Delta$, which includes the situation $\Delta \sim \Gamma$.) For $m \Gamma \sim Q \Delta$ we can formulate the multipole expansion for the coupling of soft gluons to the heavy quarks by splitting the momenta into a large label components of size $Q \Gamma / m$ and $\Gamma$, and residual momentum components of size $m \Gamma / Q$. Thus ${ }^{3}$

$$
\begin{aligned}
& i \tilde{\partial}_{+}^{\mu}=\frac{n^{\mu}}{2} \bar{n} \cdot \mathcal{P}_{c}+\mathcal{P}_{c \perp}^{\mu}+\frac{\bar{n}^{\mu}}{2} n \cdot i \partial, \\
& i \tilde{\partial}_{-}^{\mu}=\frac{\bar{n}^{\mu}}{2} n \cdot \mathcal{P}_{c}+\mathcal{P}_{c \perp}^{\mu}+\frac{n^{\mu}}{2} \bar{n} \cdot i \partial .
\end{aligned}
$$

The notation indicates that soft momenta only appear in the components $i \partial^{\mu}$. On the other hand ultracollinear momenta appear in all four components and are picked out by the label operators $\mathcal{P}_{c}^{\mu}$ or the $i \partial$. Next we make the same field redefinition on bHQET fields that we made on the SCET fields in Eq. (19)

$$
\begin{array}{ll}
h_{v_{+}} \rightarrow Y_{n} h_{v_{+}}, & A_{+}^{\mu} \rightarrow Y_{n} A_{+}^{\mu} Y_{n}^{\dagger}, \\
h_{v_{-}} \rightarrow Y_{\bar{n}} h_{v_{-}}, & A_{-}^{\mu} \rightarrow Y_{n} A_{-}^{\mu} Y_{n}^{\dagger},
\end{array}
$$

where the fields in $Y_{n}$ and $Y_{\bar{n}}$ are soft gluons. Since

$$
\begin{aligned}
& \left(v_{+} \cdot \bar{n}\right)\left(i n \cdot \partial+g n \cdot A_{s}\right) Y_{n}=0, \\
& \left(v_{-} \cdot n\right)\left(i \bar{n} \cdot \partial+g \bar{n} \cdot A_{s}\right) Y_{\bar{n}}=0,
\end{aligned}
$$

this field redefinition gives back exactly Eq. (33) for the bHQET Lagrangian and also gives a leading ucollinear

\footnotetext{
${ }^{3}$ This formulation of the multipole expansion is the same as for the coupling of ultrasoft particles to collinear particles in SCET [28] where the two types of derivatives are formally separated by introducing label operators and leaving residual momenta to be picked out by $i \partial^{\mu}$.
}

gluon action that has no couplings to soft gluons. In addition when making the field redefinition in the bHQET currents, Eq. (37), we get exactly the same soft cross talk matrix element for the two-jet production

$$
\left\langle 0\left|\left(\bar{Y}_{\bar{n}}\right)^{b a}\left(Y_{n}\right)^{b c}\right| X_{s}\right\rangle\left\langle X_{s}\left|\left(Y_{n}^{\dagger}\right)^{c d}\left(\bar{Y}_{\bar{n}}^{\dagger}\right)^{a d}\right| 0\right\rangle .
$$

The only difference between the SCET matrix element in Eq. (30) and the HQET matrix element in Eq. (41) is that in the former the soft gluons couple to the mass modes $q_{m}$ fields, while there are no such couplings in the latter. In matching renormalized soft matrix elements at a scale $\mu \simeq$ $m$

the only effect of these couplings to $q_{m}$ fields is to induce an overall Wilson coefficient, so that $S^{\text {SCET }}=$ $T_{0}(m, \mu) S^{\text {bHQET }}$. Thus the main dynamics of the soft gluons is not modified in a substantial way by passing from SCET to the boosted HQET Lagrangian, nor by the presence of the width term for unstable quarks.

For completeness, let us now consider the case $\Delta \gg$ $m \Gamma / Q$ and show that the same result is obtained. In this case a soft gluon of momentum $\ell^{\mu}$, coupling to an $h_{v_{+}}$with residual momentum $k_{+}$, has $\bar{n} \cdot \ell \gg \bar{n} \cdot k_{+}$, while for $h_{v}$ we have $n \cdot \ell \gg n \cdot k_{-}$. Thus these soft gluons knock the heavy quarks away from their mass shell, and their interactions cannot be formulated in a local manner in the same theory as the ucollinear gluons. This is similar to how soft and collinear gluons interact in the theory SCET $_{\text {II }}$ as discussed in Ref. [28]. To derive the form of the soft gluon interactions for this situation we can construct an auxiliary intermediate theory where the ucollinear gluons and heavy quarks are further from their mass shell and the soft interactions are local. The form of this theory is identical to Eqs. (33), (36), and (38), but with $\Gamma$ in Eq. (34) replaced by $Q \Delta / \mathrm{m}$, and we can make the field redefinition of Eq. (39) in this theory. Then we lower the off-shellness of the ucollinear particles and match onto the bHQET with scaling exactly as in Eq. (34). (This is identical to the procedure used to construct $\mathrm{SCET}_{\mathrm{II}}$ operators from SCET $_{\text {I }}$ which was devised in Refs. [35,62].) The result of this procedure is exactly Eqs. (33) and (41). Thus, the result for $\Delta \gg m \Gamma / Q$ is the same as for $\Delta \sim m \Gamma / Q$.

We conclude that at leading order the interaction of the bHQET heavy quarks with soft gluons are described by Eq. (41). This matrix element can be used to define a soft function $S$, that describes the cross talk between massive top quarks which have fluctuations below the mass scale $m$, and we can use Eq. (33) for the remaining dynamics at leading order. Thus, the dynamics separates in the manner shown in Fig. 2, into two decoupled HQET's and a decoupled soft sector. In Sec. III F below we will derive the same result in an alternative manner, starting from the factorization theorem for the cross section in SCET. In this approach the jet functions and soft cross talk matrix elements are first defined in SCET, and then matched onto bHQET. In this case the soft couplings are fully formulated 
by the matrix element in Eq. (41), and there is no need to consider soft couplings to fields in the bHQET Lagrangian.

Decay product interactions. - It is conspicuous that in the leading order bHQET setup, gluon exchange involving top and antitop decay products is not present. We now show that this treatment is correct and discuss the size of possible power corrections. Since we are interested in top/ antitop invariant masses in the peak region at large $Q$, we only have to consider ucollinear and soft gluons. Concerning ucollinear gluons it is convenient to switch for each bHQET into the respective heavy quark rest frame where $v_{ \pm}^{\mu}=(1,0,0,0)$ and the ucollinear gluons have momenta $k^{\mu} \sim \Gamma \ll m$. For the hemisphere invariant masses we can treat the top decay as fully inclusive at leading order (see Sec. III), so we can address the issue by analyzing possible cuts from the top/antitop final states in electroweak diagrams contributing to the bHQET matching conditions [19]. At leading order in the expansion in $\Gamma / m$ there are cuts from the top/antitop self-energy which lead to the width terms in $\mathcal{L}_{+}$. Subleading finite lifetime corrections to the heavy quark bilinear terms are suppressed by $\Gamma / m$ and physically related to the lifetimedilations coming from residual momentum fluctuations of the heavy quark. Furthermore, due to gauge invariance finite lifetime matching contributions cannot arise for the $v_{ \pm} \cdot A_{ \pm}$couplings in the covariant derivatives of $\mathcal{L}_{ \pm}$. Diagrammatically this involves a cancellation between the graphs in Fig. 4 including all possible cuts. Diagram (a) is a vertex correction, while diagrams (b) and (c) are wave-function-type contributions. Since momenta in the cut graphs are of order $m$, at leading order we can take the ucollinear gluons to have momentum $k^{\mu}=0$. In this situation the diagrams cancel due to gauge invariance. Thus, at leading order there are no finite lifetime effects involving ucollinear gluon exchange. Effects from the sum of the diagrams in Fig. 4 that do not cancel are suppressed by at least a factor $\alpha_{s} \Gamma / m$ relative to the leading order factorization theorem.

Finally we consider soft gluon interactions. Using the proof above for the universality of the soft cross talk matrix element in Eq. (41) and repeating the arguments made for the ucollinear gluon interactions we find that the dominant soft gluon interactions involving top/antitop decay products are described by possible cuts of electroweak matching contributions of the $n \cdot A_{s}$ and $\bar{n} \cdot A_{s}$ couplings in Eq. (36). In this case the same cancellation as for the ucollinear gluons takes place since the average soft gluon energy in the top/antitop rest frame is still $\Delta$ and thus much smaller than $m$. Thus interactions involving top/antitop decays products and soft gluons are suppressed by at least a factor $\Delta / m$. Numerical studies in Ref. [63] have estimated QCD interconnection effects based on nonperturbative models (see also Ref. [64]).

Having defined the EFT's we now turn to the derivation of the factorization theorem.

\section{FACTORIZED CROSS SECTION AND INVARIANT MASS DEFINITIONS}

\section{A. The QCD cross section}

We start with the general expression of the cross section for top-antitop quark production, $e^{+} e^{-} \rightarrow \gamma^{*}, Z^{*} \rightarrow t \bar{t}+$ $X$. The final state we are interested in is observed as the top and antitop jets plus soft radiation $J(t) J(\vec{t}) X_{s}$. We remind the reader that we refer to all the jets coming from the top and antitop quark decay collectively as top and antitop jets, respectively. But we stress that despite the language, our analysis is still perfectly consistent with the fact that the different jets from each the top and antitop decay can be resolved in the experimental analysis.

The full cross section is

$$
\begin{aligned}
\sigma= & \sum_{X}^{\text {res. }}(2 \pi)^{4} \delta^{4}\left(q-p_{X}\right) \sum_{i=a, v} L_{\mu \nu}^{i}\left\langle 0\left|\mathcal{J}_{i}^{\nu \dagger}(0)\right| X\right\rangle \\
& \times\left\langle X\left|\mathcal{J}_{i}^{\mu}(0)\right| 0\right\rangle,
\end{aligned}
$$

where the initial state total leptonic momentum is $q=$ $p_{e^{-}}+p_{e^{+}}, Q^{2}=q^{2}$, and the QCD currents $\mathcal{J}_{v, a}^{\mu}$ are given in Eqs. (16). The superscript res. on the summation symbol denotes a restriction on the sum over final states $X$, to give $J(t) J(\bar{t}) X_{s}$. These final states contain top and antitop jets with invariant masses close to the top quark mass. The explicit form of these restrictions depends on the specific jet and invariant mass definitions used. For the hemisphere invariant mass prescription these restrictions will be implemented explicitly in Sec. III E below, while other methods are discussed in Sec. V.

In Eq. (42) we include photon and $Z$ boson exchange, and imply an angular average of the leptonic tensor, to obtain the parity conserving $L_{\mu \nu}^{i}$ with a sum over vector and axial-vector parts, $i=v, a$. For convenience we also include the charges and boson propagators, and the cross section prefactor $1 /\left(2 Q^{2}\right)$, so that

$$
\begin{aligned}
L_{\mu \nu}^{(v)}= & -\frac{8 \pi^{2} \alpha^{2}}{3 Q^{4}}\left(g_{\mu \nu}-\frac{q_{\mu} q_{\nu}}{Q^{2}}\right) \\
& \times\left[e_{t}^{2}-\frac{2 Q^{2} v_{e} v_{t} e_{t}}{Q^{2}-m_{Z}^{2}}+\frac{Q^{4}\left(v_{e}^{2}+a_{e}^{2}\right) v_{t}^{2}}{\left(Q^{2}-m_{Z}^{2}\right)^{2}}\right], \\
L_{\mu \nu}^{(a)}= & -\frac{8 \pi^{2} \alpha^{2}}{3 Q^{4}}\left(g_{\mu \nu}-\frac{q_{\mu} q_{\nu}}{Q^{2}}\right)\left[\frac{Q^{4}\left(v_{e}^{2}+a_{e}^{2}\right) a_{t}^{2}}{\left(Q^{2}-m_{Z}^{2}\right)^{2}}\right] .
\end{aligned}
$$

Here $e_{t}$ is the top quark charge, and

$$
v_{f}=\frac{T_{3}^{f}-2 Q_{f} \sin ^{2} \theta_{W}}{2 \sin \theta_{W} \cos \theta_{W}}, \quad a_{f}=\frac{T_{3}^{f}}{2 \sin \theta_{W} \cos \theta_{W}},
$$

where $T_{3}^{f}$ is the third component of weak isospin, and $\theta_{W}$ is the weak mixing angle. 


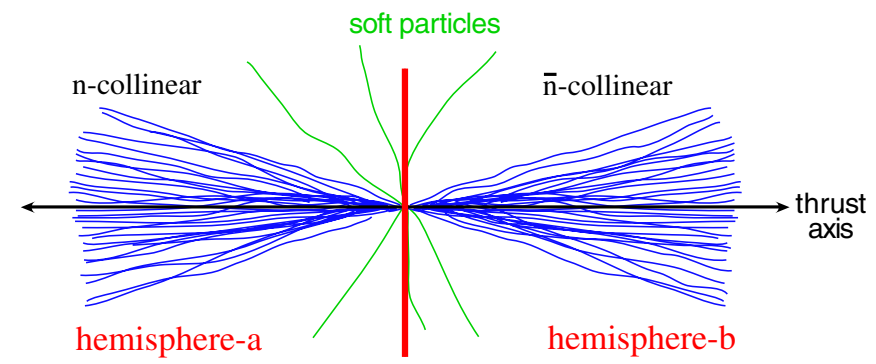

FIG. 3 (color online). Final-state jets in SCET for stable top quarks with invariant mass $\sim m^{2}$. The invariant mass is restricted and the top decay products become explicit by matching onto HQET.

\section{B. The SCET cross section}

We now proceed by using the fact that the states are restricted to be dijetlike through the constraint that the top and antitop jet invariant masses are close to the top quark mass, as illustrated in Fig. 3. In this section we reformulate the cross section by using the more specific SCET currents of Eq. (18) that are suitable for this kinematic situation. We integrate out the hard production energy scale $Q$ by matching the SCET currents onto the QCD currents giving us via the matching relation (17) a new expression for the cross section defined with matrix elements in SCET.

The SCET currents in Eq. (18) correctly reproduce the long distance physics of the QCD current, and the difference in the short-distance physics is contained in the Wilson coefficient $C(\omega, \bar{\omega}, \mu)$. We will see momentarily that momentum conservation dictates that the final form of the cross section depends only on $C(Q,-Q, \mu) \equiv$ $C(Q, \mu)$. In Ref. [65] the Wilson coefficient at one-loop was computed. It is independent of the Dirac structure $\Gamma_{i}$ and also of whether or not the collinear quarks are massive (the latter fact is demonstrated in Ref. [66] where the matching computation for the corresponding vertex diagrams is carried out explicitly for finite heavy quark mass). The result is

$$
\begin{aligned}
C(Q, \mu)= & 1+\frac{\alpha_{s} C_{F}}{4 \pi}\left[3 \log \frac{-Q^{2}-i 0}{\mu^{2}}\right. \\
& \left.-\log ^{2} \frac{-Q^{2}-i 0}{\mu^{2}}-8+\frac{\pi^{2}}{6}\right] .
\end{aligned}
$$

At the matching scale $\mu=Q$ this Wilson coefficient does not contain any large logarithms. The product of the
Wilson coefficient $C(Q, \mu)$ and the SCET matrix element is independent of the scale $\mu$, and renormalization group evolution determines the Wilson coefficient at a lower scale $\mu$. This renormalization group (RG) evolution of the hard Wilson coefficient sums logarithms of $\mu / Q$ with $\mu \gtrsim m$. The Wilson coefficient contains an imaginary part that arises from real QCD intermediates states in the QCD vertex diagram that are not accounted for in the corresponding SCET diagrams when the collinear action only contains the two sectors for the $n$ and $\bar{n}$ directions (see Sec. II A). However, only $|C(Q, \mu)|^{2}$ will appear in the final factorization theorem since we will sum over $\vec{n}$.

Using Eqs. (17) and (18) in Eq. (42), the cross section in SCET takes the form

$$
\begin{aligned}
\sigma= & \sum_{\vec{n}} \sum_{X_{n} X_{\bar{n}} X_{s}}^{\text {res. }}(2 \pi)^{4} \delta^{4}\left(q-P_{X_{n}}-P_{X_{\bar{n}}}-P_{X_{s}}\right) \\
& \times \sum_{i} L_{\mu \nu}^{(i)} \int d \omega d \bar{\omega} d \omega^{\prime} d \bar{\omega}^{\prime} C(\omega, \bar{\omega}) C^{*}\left(\omega^{\prime}, \bar{\omega}^{\prime}\right) \\
& \times\left\langle 0\left|\mathrm{~T}\left\{\bar{\chi}_{\bar{n}, \bar{\omega}^{\prime}} S_{\bar{n}}^{\dagger} \bar{\Gamma}_{i}^{\nu} S_{n} \chi_{n, \omega^{\prime}}\right\}\right| X_{n} X_{\bar{n}} X_{s}\right\rangle \\
& \times\left\langle X_{n} X_{\bar{n}} X_{s}\left|\mathrm{~T}\left\{\bar{\chi}_{n, \omega} S_{n}^{\dagger} \Gamma_{i}^{\mu} S_{\bar{n}} \chi_{\bar{n}, \bar{\omega}}\right\}\right| 0\right\rangle .
\end{aligned}
$$

Here we have pulled out the explicit sum over the top jet label directions $\vec{n}$ and keep only two collinear sectors $\mathcal{L}_{n}^{(0)}$ and $\mathcal{L}_{\bar{n}}^{(0)}$ for the SCET description of top and antitop jets. This allows us to explicitly carry out the integral over the top jet directions $\vec{n}$ in Sec. III D in parallel to implementing factorization.

In Eq. (46) we have decomposed the final states $|X\rangle$ into a soft sector $\left|X_{s}\right\rangle$ and collinear sectors $\left|X_{n}\right\rangle,\left|X_{\bar{n}}\right\rangle$ in the $\vec{n}$ and $\overrightarrow{\vec{n}}$ directions, respectively,

$$
|X\rangle=\left|X_{n} X_{\bar{n}} X_{s}\right\rangle
$$

Since the hard production scale is integrated out by the matching procedure, these states now form a complete set of final states that can be produced by the SCET currents $\mathcal{J}_{i}^{\mu}$. This already implements part of the restrictions, "res.", in the sum over states in Eq. (46). The momentum $P_{X}$ of the final state $|X\rangle$ is also decomposed into the momentum of the collinear and soft sectors:

$$
P_{X}=P_{X_{n}}+P_{X_{\bar{n}}}+P_{X_{s}} .
$$

Recall that there are no particles with $p_{m}^{\mu} \sim(m, m, m)$ scaling that can cross the final-state cut, without taking
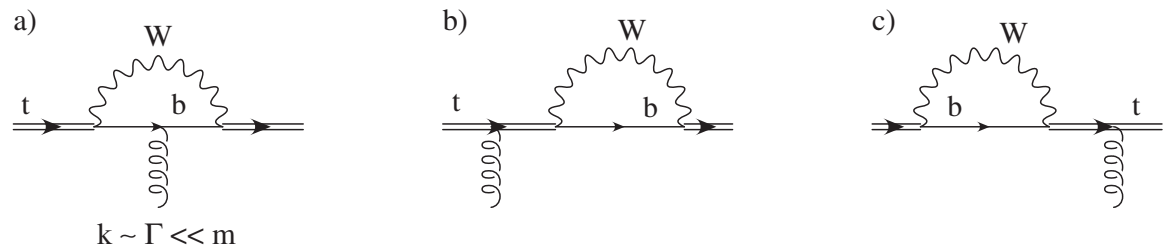

FIG. 4. Example of the cancellation of soft gluon attachments to the top decay products. 
the invariant mass far from the peak region, so there are no mass modes in this decomposition. Because the set of hadrons observed in the detector has a well-defined set of momenta, it is possible to impose criteria on the hadrons in the final state to associate them with one of $X_{n}, X_{\bar{n}}$, or $X_{s}$. Thus, the hadronic two-jet state factorizes as a direct product

$$
|X\rangle=\left|X_{n}\right\rangle\left|X_{\bar{n}}\right\rangle\left|X_{s}\right\rangle
$$

This factorization is also a manifest property of the hadronic states in SCET.
For quark and gluon states in SCET the difference from the purely hadronic case in Eq. (49) is that the states can carry global color quantum numbers. After having made the soft collinear decoupling field redefinition, the individual Lagrangians for these sectors are decoupled, and they only organize themselves into color singlets in the matrix elements which appear in the observable cross section. We can take this as a manifestation of quark-hadron duality. Using the soft collinear decoupling property from Sec. II A we can write the matrix elements in Eq. (46) as $\mathcal{M}(m, \mu)$ times

$$
\begin{aligned}
& \left\langle 0\left|\bar{\chi}_{\bar{n}, \bar{\omega}^{\prime}}^{a}\left(\bar{Y}_{\bar{n}}\right)^{b a}\left(\bar{\Gamma} Y_{n} \chi_{n, \omega^{\prime}}\right)^{b}\right| X_{n} X_{\bar{n}} X_{s}\right\rangle\left\langle X_{n} X_{\bar{n}} X_{s}\left|\left(\bar{\chi}_{n, \omega} Y_{n}^{\dagger} \Gamma\right)^{c}\left(\bar{Y}_{\bar{n}}^{\dagger}\right)^{d c} \chi_{\bar{n}, \bar{\omega}}^{d}\right| 0\right\rangle \\
& \quad=\left\langle 0\left|\bar{\chi}_{\bar{n}, \bar{\omega}^{\prime}}^{a}\right| X_{\bar{n}}\right\rangle\left\langle X_{\bar{n}}\left|\chi_{\bar{n}, \bar{\omega}}^{a^{\prime}}\right| 0\right\rangle\left\langle 0\left|\chi_{n, \omega^{\prime}}^{b}\right| X_{n}\right\rangle\left\langle X_{n}\left|\bar{\chi}_{n, \omega}^{b^{\prime}}\right| 0\right\rangle\left\langle 0\left|\left(\bar{Y}_{\bar{n}}\right)^{c a}\left(\bar{\Gamma} Y_{n}\right)^{c b}\right| X_{s}\right\rangle\left\langle X_{s}\left|\left(Y_{n}^{\dagger} \Gamma\right)^{b^{\prime} c^{\prime}}\left(\bar{Y}_{\bar{n}}^{\dagger}\right)^{a^{\prime} c^{\prime}}\right| 0\right\rangle,
\end{aligned}
$$

where here roman indices are for color and spin and $\left|X_{n}\right\rangle$ and $\left|X_{\bar{n}}\right\rangle$ are color triplets. Next we rearrange the color and spinor indices so that they are fully contracted within each of the $n$-collinear, $\bar{n}$-collinear, and soft product of matrix elements. This makes explicit the fact that in SCET each of these contributions to the cross section must separately be a spin and color singlet. Although it is not absolutely necessary to make this arrangement of indices manifest at this point, it does allow us to avoid carrying around unnecessary indices (a similar manipulation was used for $B \rightarrow X_{s} \gamma$ in Ref. [67]). For color, our $\left|X_{\bar{n}}\right\rangle\left\langle X_{\bar{n}}\right|$ forces the indices on $\bar{\chi}_{\bar{n}}^{a}$ and $\chi_{\bar{n}}^{a^{\prime}}$ to be the same, so $\left\langle 0\left|\bar{\chi}_{\bar{n}}^{a}\right| X_{\bar{n}}\right\rangle\left\langle X_{\bar{n}}\left|\chi_{\bar{n}}^{a^{\prime}}\right| 0\right\rangle=\left(\delta^{a a^{\prime}} / N_{c}\right)\left\langle 0\left|\bar{\chi}_{\bar{n}}^{b}\right| X_{\bar{n}}\right\rangle\left\langle X_{\bar{n}}\left|\chi_{\bar{n}}^{b}\right| 0\right\rangle$. A similar result holds for the $n$-collinear matrix elements. For spin we can use the SCET Fierz formula

$$
1 \otimes 1=\frac{1}{2}\left[\left(\frac{\bar{h}}{2}\right) \otimes\left(\frac{\not h}{2}\right)+\left(\frac{-\bar{h} \gamma_{5}}{2}\right) \otimes\left(\frac{\not h \gamma_{5}}{2}\right)+\left(\frac{-\bar{h} \gamma_{\perp}^{\alpha}}{2}\right) \otimes\left(\frac{\not h \gamma_{\alpha}^{\perp}}{2}\right)\right]
$$

which is valid when the identity matrices are inserted so that the $\not h$ terms on the right-hand side appear between $\bar{\chi}_{\bar{n}} \cdots \chi_{\bar{n}}$ without additional $\bar{h}$ factors next to these fields (or the analogous statement with $n \leftrightarrow \bar{n}$ ). Combining the color and spin index rearrangement, the matrix element in Eq. (50) becomes

$$
\begin{aligned}
\operatorname{tr} & {\left[\frac{\not h}{2} \Gamma_{i}^{\mu} \frac{\bar{h}}{2} \bar{\Gamma}_{i}^{\nu}\right]\left[\left\langle 0\left|\bar{\chi}_{\bar{n}, \bar{\omega}^{\prime}}^{a}\right| X_{\bar{n}}\right\rangle\left\langle X_{\bar{n}}\left|\left(\frac{\not h}{4 N_{c}} \chi_{\bar{n}, \bar{\omega}}\right)^{a}\right| 0\right\rangle\right]\left[\left\langle 0\left|\left(\frac{\bar{h}}{4 N_{c}} \chi_{n, \omega^{\prime}}\right)^{b}\right| X_{n}\right\rangle\left\langle X_{n}\left|\bar{\chi}_{n, \omega}^{b}\right| 0\right\rangle\right] } \\
& \times\left[\left\langle 0\left|\left(\bar{Y}_{\bar{n}}\right)^{c a^{\prime}}\left(Y_{n}\right)^{c b^{\prime}}\right| X_{s}\right\rangle\left\langle X_{s}\left|\left(Y_{n}^{\dagger}\right)^{b^{\prime} c^{\prime}}\left(\bar{Y}_{\bar{n}}^{\dagger}\right)^{a^{\prime} c^{\prime}}\right| 0\right\rangle\right] \\
\equiv & \operatorname{tr}\left[\frac{\not h}{2} \Gamma_{i}^{\mu} \frac{\bar{h}}{2} \bar{\Gamma}_{i}^{\nu}\right] \operatorname{tr}\left(\left\langle 0\left|\bar{\chi}_{\bar{n}, \bar{\omega}^{\prime}}\right| X_{\bar{n}}\right\rangle\left\langle X_{\bar{n}}\left|\hat{h} \chi_{\bar{n}, \bar{\omega}}\right| 0\right\rangle\right) \operatorname{tr}\left(\left\langle 0\left|\hat{\bar{h}} \chi_{n, \omega^{\prime}}\right| X_{n}\right\rangle\left\langle X_{n}\left|\bar{\chi}_{n, \omega}\right| 0\right\rangle\right) \times \operatorname{tr}\left(\left\langle 0\left|\bar{Y}_{\bar{n}} Y_{n}\right| X_{s}\right\rangle\left\langle X_{s}\left|Y_{n}^{\dagger} \bar{Y}_{\bar{n}}^{\dagger}\right| 0\right\rangle\right),
\end{aligned}
$$

where for convenience we defined

$$
\hat{h} \equiv \not h /\left(4 N_{c}\right), \quad \hat{\vec{h}} \equiv \bar{h} /\left(4 N_{c}\right) .
$$

Note that only the first term on the right-hand side of Eq. (51) contributes because the collinear states give at least one matrix element which is zero when we have a $\gamma_{5}$ or $\gamma_{\perp}^{\alpha}$. This factorizes the SCET cross section into a product of three singlets under spin and color. For convenience we will in the following suppress writing these explicit traces on the matrix elements.

Using Eq. (52) in Eq. (46), the factorized SCET cross section takes the form

$$
\begin{aligned}
\sigma= & K_{0} \mathcal{M} \sum_{\bar{n}} \sum_{X_{n} X_{\bar{n}} X_{s}}^{\text {res. }}(2 \pi)^{4} \delta^{4}\left(q-P_{X_{n}}-P_{X_{\bar{n}}}-P_{X_{s}}\right) \\
& \times\left\langle 0\left|\bar{Y}_{\bar{n}} Y_{n}\right| X_{s}\right\rangle\left\langle X_{s}\left|Y_{n}^{\dagger} \bar{Y}_{\bar{n}}^{\dagger}\right| 0\right\rangle \int d \omega d \bar{\omega} d \omega^{\prime} d \bar{\omega}^{\prime} C(\omega, \bar{\omega}) \\
& \times C^{\dagger}\left(\omega^{\prime}, \bar{\omega}^{\prime}\right)\left\langle 0\left|\hat{\bar{h}} \chi_{n, \omega^{\prime}}\right| X_{n}\right\rangle\left\langle X_{n}\left|\bar{\chi}_{n, \omega}\right| 0\right\rangle \\
& \times\left\langle 0\left|\bar{\chi}_{\bar{n}, \bar{\omega}^{\prime}}\right| X_{\bar{n}}\right\rangle\left\langle X_{\bar{n}}\left|\hat{\not} \chi_{\bar{n}, \bar{\omega}}\right| 0\right\rangle
\end{aligned}
$$

where $\mathcal{M}=\mathcal{M}(m, \mu)$ and we defined the normalization factor 


$$
\begin{aligned}
K_{0} & =\sum_{i=v, a} L_{\mu \nu}^{(i)} \operatorname{Tr}\left[\frac{\not h}{2} \Gamma_{i}^{\mu} \frac{\bar{h}}{2} \bar{\Gamma}_{i}^{\nu}\right]=-2 g_{\perp}^{\mu \nu} \sum_{i=v, a} L_{\mu \nu}^{(i)} \\
& =\frac{32 \pi^{2} \alpha^{2}}{3 Q^{4}}\left[e_{t}^{2}-\frac{2 Q^{2} v_{e} v_{t} e_{t}}{Q^{2}-m_{Z}^{2}}+\frac{Q^{4}\left(v_{e}^{2}+a_{e}^{2}\right)\left(v_{t}^{2}+a_{t}^{2}\right)}{\left(Q^{2}-m_{Z}^{2}\right)^{2}}\right] .
\end{aligned}
$$

We can further simplify the form of the factorized cross section. First we use the identities

$$
\begin{aligned}
& \left\langle X_{n}\left|\bar{\chi}_{n, \omega^{\prime}}\right| 0\right\rangle=\left\langle X_{n}\left|\bar{\chi}_{n} \delta_{\omega^{\prime}, \bar{n} \cdot \mathcal{P}^{\dagger}}\right| 0\right\rangle=\delta_{\omega^{\prime}, p_{X_{n}}^{-}}\left\langle X_{n}\left|\bar{\chi}_{n}\right| 0\right\rangle, \\
& \left\langle X_{\bar{n}}\left|\bar{\chi}_{\bar{n}, \bar{\omega}^{\prime}}\right| 0\right\rangle=\left\langle X_{\bar{n}}\left|\bar{\chi}_{\bar{n}} \delta_{\bar{\omega}^{\prime}, n \cdot \mathcal{P}^{\dagger}}\right| 0\right\rangle=\delta_{-\bar{\omega}^{\prime}, p_{X_{\bar{n}}^{+}}^{+}}\left\langle X_{\bar{n}}\left|\bar{\chi}_{\bar{n}}\right| 0\right\rangle,
\end{aligned}
$$

with similar relations for the other two collinear matrix elements in Eq. (54). Combining this with the relation $\delta_{\omega^{\prime}, p_{X_{n}}^{-}} \delta_{\omega, p_{X_{n}}^{-}}=\delta_{\omega^{\prime}, \omega} \delta_{\omega, p_{X_{n}}^{-}}$, and analog for $p_{X_{\bar{n}}}^{+}$, we can write the product of collinear matrix elements in Eq. (54) as

$$
\begin{aligned}
& \left\langle 0\left|\hat{\vec{h}} \chi_{n, \omega^{\prime}}\right| X_{n}\right\rangle\left\langle X_{n}\left|\bar{\chi}_{n, \omega}\right| 0\right\rangle\left\langle 0\left|\bar{\chi}_{\bar{n}, \bar{\omega}^{\prime}}\right| X_{\bar{n}}\right\rangle\left\langle X_{\bar{n}}\left|\hat{\not} \chi_{\bar{n}, \bar{\omega}}\right| 0\right\rangle \\
& =\delta_{\bar{\omega}^{\prime}, \bar{\omega}} \delta_{\omega^{\prime}, \omega}\left\langle 0\left|\hat{\vec{h}} \chi_{n}\right| X_{n}\right\rangle\left\langle X_{n}\left|\bar{\chi}_{n, \omega}\right| 0\right\rangle\left\langle 0\left|\bar{\chi}_{\bar{n}}\right| X_{\bar{n}}\right\rangle \\
& \quad \times\left\langle X_{\bar{n}}\left|\hat{h} \chi_{\bar{n}, \bar{\omega}}\right| 0\right\rangle .
\end{aligned}
$$

Next we do the sums over $\omega^{\prime}, \bar{\omega}^{\prime}$ to arrive at the form

$$
\begin{aligned}
\sigma= & K_{0} \mathcal{M} \sum_{\bar{n}} \sum_{X_{n} X_{\bar{n}} X_{s}}^{\text {res. }}(2 \pi)^{4} \delta^{4}\left(q-P_{X_{n}}-P_{X_{\bar{n}}}-P_{X_{s}}\right) \\
& \times\left\langle 0\left|\bar{Y}_{\bar{n}} Y_{n}\right| X_{s}\right\rangle\left\langle X_{s}\left|Y_{n}^{\dagger} \bar{Y}_{\bar{n}}^{\dagger}\right| 0\right\rangle \int d \omega d \bar{\omega}|C(\omega, \bar{\omega})|^{2} \\
& \times\left\langle 0\left|\hat{\bar{h}} \chi_{n}\right| X_{n}\right\rangle\left\langle X_{n}\left|\bar{\chi}_{n, \omega}\right| 0\right\rangle\left\langle 0\left|\bar{\chi}_{\bar{n}}\right| X_{\bar{n}}\right\rangle\left\langle X_{\bar{n}}\left|\hat{h} \chi_{\bar{n}, \bar{\omega}}\right| 0\right\rangle .
\end{aligned}
$$

Before proceeding, we pause to define the thrust axis which is needed to properly define the invariant mass of jets and to state its relation to the direction of the energetic collinear degrees of freedom. Then in order to make the power counting manifest we decompose the final-state momenta into label and residual parts and perform some general manipulations of the phase space integrals to setup a formula for the cross section to be used for the remaining calculation.

\section{Thrust or jet axis}

The thrust $T$ of any event is defined to be

$$
T=\max _{\hat{\mathbf{t}}} \frac{\sum_{i}\left|\hat{\mathbf{t}} \cdot \mathbf{p}_{i}\right|}{Q},
$$

where the sum is over the momenta $\mathbf{p}_{i}$ of all the final-state particles produced. The thrust axis $\hat{\mathbf{t}}$ is chosen so that it maximizes the sum of particle momenta projected along $\hat{\mathbf{t}}$. Intuitively, for a dijetlike event the thrust axis corresponds to the axis along which most of the momentum is deposited. Conversely, the thrust $T$ is close to its maximum for a dijetlike event. We choose $\vec{n}$ to point along $\hat{\mathbf{t}}$. For an event with exactly two massive stable particles $T=$ $\sqrt{Q^{2}-4 m^{2}} / Q=1-2 m^{2} / Q^{2}+\mathcal{O}\left(m^{4} / Q^{4}\right)$ is the maximum allowed thrust. Since we are interested in thrusts in the dijet region for the top and antitop jets it is convenient to define a shifted thrust parameter,

$$
\tau=\sqrt{1-\frac{4 m^{2}}{Q^{2}}}-T=1-\frac{2 m^{2}}{Q^{2}}-T+\mathcal{O}\left(\frac{m^{4}}{Q^{4}}\right) .
$$

For stable top-antitop production additional jets always result in $\tau>0$. For unstable top quarks the values of $\tau<$ 0 also become allowed. Note that for massless jet production the thrust $(T)$ distribution is peaked close to $T=1$ while for events containing a heavy quark pair it is peaked close to $T=\sqrt{Q^{2}-4 m^{2}} / Q$. Thus a cut on thrust can in principle be used to discriminate between massive and massless quark production [9].

\section{Differential cross section with momentum decomposition}

To insert the invariant mass constraints into our cross section in Eq. (54) we use the identity operator:

$$
\begin{aligned}
1= & \int d^{4} p_{n} d^{4} p_{\bar{n}} d^{4} p_{s} \delta^{4}\left(p_{n}-P_{X_{n}}\right) \delta^{4}\left(p_{\bar{n}}-P_{X_{\bar{n}}}\right) \\
& \times \delta^{4}\left(p_{s}-P_{X_{s}}\right),
\end{aligned}
$$

which sets the total collinear and soft momenta of the states $P_{X_{n}}, P_{X_{\bar{n}}}, P_{X_{s}}$ to $p_{n}, p_{\bar{n}}, p_{s}$, respectively. In Sec. III E we will use an additional insertion of an identity operator to define the hemisphere invariant masses, $M_{t}$ and $M_{\bar{t}}$. In this section we carry out manipulations that are common to any definition of the invariant masses. For now we ensure that the invariant mass of each hemisphere is close to the top mass by including in the restrictions, res, on the states the fact that $M_{t}, M_{\bar{t}}$ are in the region

$$
\left|s_{t, \bar{t}}\right|=\left|M_{t, \bar{t}}^{2}-m^{2}\right| \ll m^{2} .
$$

From here on we assume that in the sense of power counting $\Delta \sim \Gamma$. We now decompose the collinear and soft momenta into label and residual parts

$$
\begin{gathered}
p_{n}=\tilde{p}_{n}+k_{n}, \quad p_{\bar{n}}=\tilde{p}_{\bar{n}}+k_{\bar{n}}, \quad P_{\bar{X}_{n}}^{\perp}=K_{X_{n}}^{\perp}, \\
P_{\bar{X}_{\bar{n}}}^{\perp}=K_{\bar{X}_{\bar{n}}}^{\perp}, \quad P_{X_{n}}^{-}=\tilde{P}_{X_{n}}^{-}+K_{X_{n}}^{-}, \\
P_{X_{\bar{n}}}^{+}=\tilde{P}_{X_{\bar{n}}}^{+}+K_{\bar{X}_{\bar{n}}}^{+}, \quad P_{X_{n}}^{+}=K_{X_{n}}^{+}, \quad P_{X_{\bar{n}}}^{-}=K_{\bar{X}_{\bar{n}}}^{-}, \\
P_{X_{s}}^{\mu}=K_{X_{s}}^{\mu}, \quad p_{s}^{\mu}=k_{s}^{\mu} .
\end{gathered}
$$

Note that our choice of $\vec{n}$ along the thrust axis together with the restrictions on the states ensures that the perpendicular momentum of the jets relative to this axis, $P_{X_{n}}^{\perp}$ and $P_{X_{\bar{n}}}^{\perp}$, are purely residual. The last result in Eq. (63) indicates that the soft state also has a momentum that is purely residual. The 
integrals in Eq. (61) can be decomposed into a sum over labels and integrals over residual momenta as

$$
\begin{aligned}
\int d^{4} p_{n} \int d^{4} p_{\bar{n}}= & \frac{1}{2} \sum_{\tilde{p}_{n}} \int d k_{n}^{+} d k_{n}^{-} d^{2} k_{n}^{\perp} \\
& \times \frac{1}{2} \sum_{\tilde{p}_{\bar{n}}} \int d k_{\bar{n}}^{+} d k_{\bar{n}}^{-} d^{2} k_{\bar{n}}^{\perp} .
\end{aligned}
$$

In the total cross section in Eq. (54) we sum over the directions $\vec{n}$ of the thrust axis. To turn this sum into an integral over the full solid angle, we need to combine it with a residual solid angle integration for each $\vec{n}$. Therefore, we decompose the residual measure as

$$
d^{2} k_{n}^{\perp}=\left|\vec{p}_{n}\right|^{2} d \phi d \cos \left(\theta_{r}\right)=\left(\frac{Q^{2}}{4}-p_{n}^{2}\right) d \phi d \cos \left(\theta_{r}\right),
$$

where $\theta_{r}$ is the small angle of $p_{3}$ relative to $\vec{p}$. In the first equality we used the fact that $\cos \left(\theta_{r}\right) \simeq 1$. Since we are in the peak region we can approximate $p_{n}^{2}=m^{2}$ up to small $\Gamma / m$ corrections. Combining this with the sum over $\vec{n}$ gives

$$
\sum_{\vec{n}} d^{2} k_{n}^{\perp}=\left(\frac{Q^{2}}{4}-m^{2}\right) d \phi d \cos (\theta)=\frac{Q^{2}}{4} d \Omega
$$

where in the last equality we work to leading order in $\mathrm{m}^{2} / \mathrm{Q}^{2}$. Since the angular averaged two-jet production is independent of the thrust direction we are free to carry out the remaining integrations in a frame where $k_{n}^{\perp}=0$, and also replace $\int d \Omega=4 \pi$. The differential cross section now reads

$$
\begin{aligned}
& \sigma=\frac{\pi Q^{2} K_{0}}{4} \mathcal{M} \sum_{X_{n} X_{\bar{n}} X_{s}}^{\text {res. }}(2 \pi)^{4} \delta^{4}\left(q-P_{X_{n}}-P_{X_{\bar{n}}}-P_{X_{s}}\right) \sum_{\tilde{p}_{n}, \tilde{p}_{\bar{n}}} \int d k_{n}^{+} d k_{n}^{-} \int d k_{\bar{n}}^{+} d k_{\bar{n}}^{-} d^{2} k_{\bar{n}}^{\perp} d^{4} k_{s} \delta^{4}\left(p_{n}-P_{X_{n}}\right) \\
& \times \delta^{4}\left(p_{\bar{n}}-P_{X_{\bar{n}}}\right) \delta^{4}\left(k_{s}-P_{X_{s}}\right)\left\langle 0\left|\bar{Y}_{\bar{n}} Y_{n}\right| X_{s}\right\rangle\left\langle X_{s}\left|Y_{n}^{\dagger} \bar{Y}_{\bar{n}}^{\dagger}\right| 0\right\rangle \int d \omega d \bar{\omega}|C(\omega, \bar{\omega})|^{2}\left\langle 0\left|\hat{\bar{h}} \chi_{n}(0)\right| X_{n}\right\rangle\left\langle X_{n}\left|\bar{\chi}_{n, \omega}(0)\right| 0\right\rangle\left\langle 0\left|\bar{\chi}_{\bar{n}}(0)\right| X_{\bar{n}}\right\rangle \\
& \times\left\langle X_{\bar{n}}\left|\hat{h} \chi_{\bar{n}, \bar{\omega}}(0)\right| 0\right\rangle .
\end{aligned}
$$

In the remainder of this section we will simplify this formula as much as possible prior to specifying the exact constraints on the restricted sum of states. First we decompose the delta functions into label and residual parts as

$$
\begin{aligned}
& \delta^{4}\left(p_{n}-P_{X_{n}}\right)=\delta_{\tilde{p}_{n}, \tilde{P}_{X_{n}}} \delta^{4}\left(k_{n}-K_{X_{n}}\right)=\delta_{\tilde{p}_{n}^{-}, \omega} \delta_{\tilde{p}_{n}^{\perp}, 0} \int \frac{d^{4} x}{(2 \pi)^{4}} e^{i\left[\left(k_{n}^{+}-K_{X_{n}}^{+}\right)\left(x^{-} / 2\right)+\left(k_{n}^{-}-K_{X_{n}}^{-}\right)\left(x^{+} / 2\right)-K_{X_{n}}^{\perp} \cdot x^{\perp}\right]}, \\
& \delta^{4}\left(p_{\bar{n}}-P_{X_{\bar{n}}}\right)=\delta_{\tilde{p}_{\bar{n}}, \tilde{P}_{X_{\bar{n}}}} \delta^{4}\left(k_{\bar{n}}-K_{X_{\bar{n}}}\right)=\delta_{\tilde{p}_{\bar{n}}^{+},-\bar{\omega}} \delta_{\tilde{p}_{\bar{n}}^{\perp}, 0} \int \frac{d^{4} y}{(2 \pi)^{4}} e^{i\left(k_{\bar{n}}-K_{X_{\bar{n}}}\right) \cdot y}, \\
& \delta^{4}\left(p_{s}-P_{X_{s}}\right)=\delta^{4}\left(k_{s}-K_{X_{s}}\right)=\int \frac{d^{4} z}{(2 \pi)^{4}} e^{i\left(k_{s}-K_{X_{s}}\right) \cdot z},
\end{aligned}
$$

where there is no $k_{n}^{\perp}$ in the first line (or below) because we fixed $k_{n}^{\perp}=0$. In the second equality on lines 1 and 2 we replaced $\tilde{P}_{X_{n}}^{-}, \tilde{P}_{X_{\bar{n}}}^{+}$with the labels $\omega, \bar{\omega}$, respectively, using the momentum conservation delta functions discussed below Eq. (56). We also decompose

$$
\delta^{4}\left(q-P_{X_{n}}-P_{X_{\bar{n}}}-K_{X_{s}}\right)=\delta_{Q, \tilde{p}_{n}^{-}} \delta_{Q, \tilde{p}_{\bar{n}}^{+}} \delta^{4}\left(k_{n}+k_{\bar{n}}+k_{s}\right),
$$

where we have replaced $P_{X_{n}}, P_{X_{\bar{n}}}$ with $p_{n}, p_{\bar{n}}$ by using the delta functions in Eq. (68).

Next we use Eqs. (68) and (69) in Eq. (67) and with the exponential factors of $e^{-i K_{X_{n}} \cdot x}, e^{-i K_{X_{n}} \cdot y}$, and $e^{-i K_{X_{s}} \cdot z}$ in Eq. (68) we translate the collinear and soft fields to the positions $x, y$, and $z$, respectively. This gives

$$
\begin{aligned}
\sigma= & \frac{\pi}{(2 \pi)^{8}} \frac{Q^{2} K_{0}}{4} \mathcal{M} \sum_{X_{n} X_{\bar{n}} X_{s}}^{\text {res. }} \int d k_{n}^{+} d k_{n}^{-} d k_{\bar{n}}^{+} d k_{\bar{n}}^{-} d^{2} k_{\bar{n}}^{\perp} d^{4} k_{s} \int d^{4} x d^{4} y d^{4} z \delta^{4}\left(k_{n}+k_{\bar{n}}+k_{s}\right) \\
& \times|C(Q, \mu)|^{2} \exp \left[\frac{i}{2} k_{n}^{+} x^{-}+\frac{i}{2} k_{n}^{-} x^{+}+i k_{\bar{n}} \cdot y+i k_{s} \cdot z\right]\left\langle 0\left|\left(\bar{Y}_{\bar{n}} Y_{n}\right)(z)\right| X_{s}\right\rangle\left\langle X_{s}\left|\left(Y_{n}^{\dagger} \bar{Y}_{\bar{n}}^{\dagger}\right)(0)\right| 0\right\rangle \\
& \times\left\langle 0\left|\hat{\bar{h}} \chi_{n}(x)\right| X_{n}\right\rangle\left\langle X_{n}\left|\bar{\chi}_{n, Q}(0)\right| 0\right\rangle\left\langle 0\left|\bar{\chi}_{\bar{n}}(y)\right| X_{\bar{n}}\right\rangle\left\langle X_{\bar{n}}\left|\hat{\not} \chi_{\bar{n},-Q}(0)\right| 0\right\rangle,
\end{aligned}
$$

where here the large label momenta in the jets are fixed to be $Q, \bar{\chi}_{n, Q}=\bar{\chi}_{n} \delta_{Q, \overline{\mathcal{P}}^{\dagger}}$, and $\chi_{\bar{n},-Q}=\delta_{-Q, \mathcal{P}} \chi_{\bar{n}}$. Next we can use the fact that the $n$-collinear graphs are independent of the $k_{n}^{-}$and $k_{n}^{\perp}$, so that the above $n$-collinear matrix element is proportional to $\delta\left(x^{+}\right) \delta\left(x_{\perp}\right)$ [28]. Similarly the $\bar{n}$-collinear matrix element is $\propto \delta\left(y^{-}\right) \delta\left(y_{\perp}\right)$. It is not crucial to use these 
$\delta$-functions at this stage, but they do allow us to simplify the formula by dropping $x^{+}, x_{\perp}, y^{-}$, and $y_{\perp}$ dependence in the exponentials. Performing a few integrals we arrive at a fairly simple form for the cross section

$$
\begin{aligned}
& \sigma=\sigma_{0}|C(Q, \mu)|^{2} \mathcal{M} \int d k_{n}^{+} d k_{\bar{n}}^{-} d k_{s}^{+} d k_{s}^{-} \sum_{X_{n}}^{\text {res. }} \frac{1}{2 \pi} \int d^{4} x e^{i k_{n}^{+} x^{-} / 2}\left\langle 0\left|\hat{\bar{h}} \chi_{n}(x)\right| X_{n}\right\rangle\left\langle X_{n}\left|\bar{\chi}_{n, Q}(0)\right| 0\right\rangle \\
& \times \sum_{X_{\bar{n}}}^{\text {res. }} \frac{1}{2 \pi} \int d^{4} y e^{i k_{\bar{n}}^{-} y^{+} / 2}\left\langle 0\left|\bar{\chi}_{\bar{n}}(y)\right| X_{\bar{n}}\right\rangle\left\langle X_{\bar{n}}\left|\hat{h} \chi_{\bar{n},-Q}(0)\right| 0\right\rangle \\
& \times \sum_{X_{s}}^{\text {res. }} \frac{1}{4 N_{c}(2 \pi)^{2}} \int d z^{+} d z^{-} e^{(i / 2)\left(k_{s}^{+} z^{-}+k_{s}^{-} z^{+}\right)}\left\langle 0\left|\bar{Y}_{\bar{n}} Y_{n}\left(z^{-}, z^{+}\right)\right| X_{s}\right\rangle\left\langle X_{s}\left|Y_{n}^{\dagger} \bar{Y}_{\bar{n}}^{\dagger}(0)\right| 0\right\rangle .
\end{aligned}
$$

The result in Eq. (71) is a factorized product of Fourier transforms over $n$-collinear, $\bar{n}$-collinear, and soft matrix elements. We introduced a $1 / N_{c}$ in front of the soft matrix element in Eq. (71) and include a compensating factor $N_{c}$ in $\sigma_{0}$. This equation provides a good starting point for the derivation of any differential cross section (for massive or massless dijet events). The new normalization factor $\sigma_{0}$ is just the total Born cross section

$$
\begin{aligned}
\sigma_{0}= & N_{c} \frac{Q^{2}}{8 \pi} K_{0} \\
= & N_{c} \frac{4 \pi \alpha^{2}}{3 Q^{2}}\left[e_{t}^{2}-\frac{2 Q^{2} v_{e} v_{t} e_{t}}{Q^{2}-m_{Z}^{2}}\right. \\
& \left.+\frac{Q^{4}\left(v_{e}^{2}+a_{e}^{2}\right)\left(v_{t}^{2}+a_{t}^{2}\right)}{\left(Q^{2}-m_{Z}^{2}\right)^{2}}\right] .
\end{aligned}
$$

For massive quarks $\sigma_{0}$ depends on $\beta_{m}=\left(1-4 m^{2} / Q^{2}\right)^{1 / 2}$ through an extra multiplicative factor of $\beta_{m}\left(3-\beta_{m}^{2}\right) / 2=$ $1-6 m^{4} / Q^{4}+\ldots$ This is only a $1 \%$ correction to $\sigma_{0}$ for $Q / m \sim 5$.

To proceed further we now need to make the prescription how the $n$ - and $\bar{n}$-collinear and the soft particles enter the invariant masses $s_{t}$ and $s_{\bar{t}}$ explicit. This removes the implicit restrictions on the sums over states indicated in Eq. (71). In the next subsection we implement the prescriptions for the hemisphere jet invariant masses. In Sec. V we briefly discuss how the implementation changes for other invariant mass prescriptions.

\section{E. Factorization for hemisphere invariant masses in SCET}

In the hemisphere mass case all the final-state particles are assigned to belong to one of two hemispheres defined with respect to the thrust axis. The boundary between the two hemispheres is perpendicular to the thrust axis and centered at the $e^{+} e^{-}$collision point, see Fig. 3. Thus the top and antitop jets we consider correspond to all the particles in the respective two hemispheres and the invariant mass of each jet is defined to be the total invariant mass of all the final-state particles in each hemisphere. As we show explicitly below, the requirement that these jet invariant masses are both close to the top mass automatically restricts the final state to be dijetlike and eliminates the need to introduce any additional event-shape constraint. We stress that some mechanism to control the soft particles is absolutely crucial for establishing the factorization theorem and the unique definition of the soft function $S$. Here this is accomplished by the fact that all soft particles enter the invariant mass variables $M_{t, \bar{t}}^{2}$.

The invariant mass of each hemisphere includes contributions from both soft and collinear particles. The total momentum of the collinear particles in the $n$ hemisphere is $P_{X_{n}}$ and in the $\bar{n}$ hemisphere is $P_{X_{\bar{n}}}$. The total final-state soft momentum $K_{X_{s}}$ is split between the two hemispheres and can be divided as

$$
K_{X_{s}}=k_{s}^{a}+k_{s}^{b}
$$

where $k_{s}^{a}$ and $k_{s}^{b}$ correspond to the total momenta of all the soft partons in the $n$ and $\bar{n}$ hemispheres, respectively. It is useful to think of these hemisphere momenta as the result of hemisphere projection operators $\hat{P}_{a}, \hat{P}_{b}$ :

$$
\hat{P}_{a}\left|X_{s}\right\rangle=k_{s}^{a}\left|X_{s}\right\rangle, \quad \hat{P}_{b}\left|X_{s}\right\rangle=k_{s}^{b}\left|X_{s}\right\rangle .
$$

In other words, these projection operators act on each state $\left|X_{s}\right\rangle$, pick out the soft partons in the respective hemisphere, and add up their total momentum. Note that the eigenvalues are dependent on the state $X_{s}$, so $k_{s}^{a}=k_{s}^{a}\left[X_{s}\right]$ and $k_{s}^{b}=$ $k_{s}^{b}\left[X_{s}\right]$. We can now define the invariant mass of each jet as $\left(P_{X_{n}}+k_{s}^{a}\right)^{2}$ and $\left(P_{X_{\bar{n}}}+k_{s}^{b}\right)^{2}$ for the $n$ and $\bar{n}$ hemispheres, respectively. The delta functions $\delta^{4}\left(p_{n}-P_{X_{n}}\right) \delta^{4}\left(p_{\bar{n}}-\right.$ $\left.P_{X_{\bar{n}}}\right)$ in Eq. (67) allow us to define the jet invariant masses in terms of $p_{n}, p_{\bar{n}}$ as $\left(p_{n}+k_{s}^{a}\right)^{2}$ and $\left(p_{\bar{n}}+k_{s}^{b}\right)^{2}$ for the $n$ and $\bar{n}$ hemispheres, respectively.

Note that this implements a very simple form of a jet algorithm. For a different jet algorithm we would change the definitions of the operators $\hat{P}_{a}$ and $\hat{P}_{b}$. Running a jet algorithm in inclusive $e^{+} e^{-}$mode [68,69] each soft parton is still accounted for, having a certain probability of being assigned to either the top or the antitop invariant mass. We discuss other algorithms in Sec. V.

If the top quark were a stable particle, these invariant mass definitions would be obvious because $n$ - and $\bar{n}$-collinear particles would be fully radiated into the $n$ and $\bar{n}$ hemispheres, respectively. Because of the finite lifetime of the top quark, however, we need to convince ourselves that this invariant mass definition still works if 
the $n$ - and $\bar{n}$-collinear momenta of the top and antitop quarks, respectively, are distributed among their decay products. So let us consider the top quark in the $n$ hemisphere. Since the top rest frame is boosted with respect to the $e^{+} e^{-}$c.m. frame with a boost factor $Q / m$, top decay events can have final-state particles appearing in the $\bar{n}$ hemisphere of the antitop quark only if these final-state particles have an angle (defined in the top rest frame) smaller than $m / Q$ with respect to the antiparticle direction. On the other hand, the top spin is only about $20 \%$ polarized (for unpolarized $e^{+} e^{-}$beams and upon averaging over the directions of the thrust axis) [70], and thus the top decay products in the top rest frame are distributed isotropically to a rather good approximation. The fraction of events in this kinematical situation is therefore suppressed by $(\mathrm{m} / \mathrm{Q})^{2}$ and can be neglected at leading order in the power counting. Of course the analogous conclusion also applies to the antitop quark in the $\bar{n}$ hemisphere. So at leading order in the power counting it is consistent to employ the invariant mass definition of the previous paragraph.

The jet invariant mass definitions can be implemented into the cross section of Eq. (71) by inserting underneath the $\sum_{X_{s}}$ the identity relation

$$
\begin{aligned}
1 & =\int d M_{t}^{2} \delta\left(\left(p_{n}+k_{s}^{a}\right)^{2}-M_{t}^{2}\right) \int d M_{\bar{t}}^{2} \delta\left(\left(p_{\bar{n}}+k_{s}^{b}\right)^{2}-M_{\bar{t}}^{2}\right) \\
& =\int d M_{t}^{2} \delta\left(\left(p_{n}+k_{s}^{a}\right)^{2}-m^{2}-s_{t}\right) \int d M_{\bar{t}}^{2} \delta\left(\left(p_{\bar{n}}+k_{s}^{b}\right)^{2}-m^{2}-s_{\bar{t}}\right),
\end{aligned}
$$

where $s_{t}=s_{t}\left(M_{t}\right)$ and $s_{\bar{t}}=s_{\bar{t}}\left(M_{\bar{t}}\right)$ from Eq. (1), i.e. it should be understood that $s_{t, \bar{t}}$ are functions of $M_{t, \bar{t}}^{2}$. In the second line $m$ is defined as the pole mass. It is straightforward to switch the final result to a suitable short-distance mass definition, as we explain in Sec. III G. Decomposing the $\delta$ functions at leading order gives

$$
\begin{array}{ll}
\delta\left(\left(p_{n}+k_{s}^{a}\right)^{2}-m^{2}-s_{t}\right)=\frac{1}{Q} \delta\left(k_{n}^{+}+k_{s}^{+a}-\frac{m^{2}+s_{t}}{Q}\right), & 1=\int d \ell^{+} d \ell^{-} \delta\left(\ell^{+}-k_{s}^{+a}\right) \delta\left(\ell^{-}-k_{s}^{-b}\right) \\
\delta\left(\left(p_{\bar{n}}+k_{s}^{b}\right)^{2}-m^{2}-s_{\bar{t}}\right)=\frac{1}{Q} \delta\left(k_{\bar{n}}^{-}+k_{s}^{-b}-\frac{m^{2}+s_{\bar{t}}}{Q}\right), &
\end{array}
$$

where we set $\tilde{p}_{n}^{-}=\tilde{p}_{\bar{n}}^{+}=Q$ due to $\delta$ functions from Eq. (69). Carrying out the integration over $k_{s}^{+}$and $k_{s}^{-}$in Eq. (71) sets the arguments of the soft function to $z^{ \pm}=0$. Inserting the identity relation

the differential cross section then reads

$$
\begin{aligned}
\frac{d^{2} \sigma}{d M_{t}^{2} d M_{\bar{t}}^{2}}= & \frac{\sigma_{0}}{Q^{2}}|C(Q, \mu)|^{2} \mathcal{M} \int d k_{n}^{+} d k_{\bar{n}}^{-} d \ell^{+} d \ell^{-} \delta\left(k_{n}^{+}+\ell^{+}-\frac{m^{2}+s_{t}}{Q}\right) \delta\left(k_{\bar{n}}^{-}+\ell^{-}-\frac{m^{2}+s_{\bar{t}}}{Q}\right) \\
& \times \sum_{X_{n}} \frac{1}{2 \pi} \int d^{4} x e^{i k_{n}^{+} x^{-} / 2} \operatorname{tr}\left\langle 0\left|\hat{\bar{h}} \chi_{n}(x)\right| X_{n}\right\rangle\left\langle X_{n}\left|\bar{\chi}_{n, Q}(0)\right| 0\right\rangle \\
& \times \sum_{X_{\bar{n}}} \frac{1}{2 \pi} \int d^{4} y e^{i k_{\bar{n}}^{-} y^{+} / 2} \operatorname{tr}\left\langle 0\left|\bar{\chi}_{\bar{n}}(y)\right| X_{\bar{n}}\right\rangle\left\langle X_{\bar{n}}\left|\hat{h} \chi_{\bar{n},-Q}(0)\right| 0\right\rangle \\
& \times \sum_{X_{s}} \frac{1}{N_{c}} \delta\left(\ell^{+}-k_{s}^{+a}\right) \delta\left(\ell^{-}-k_{s}^{-b}\right) \operatorname{tr}\left\langle 0\left|\bar{Y}_{\bar{n}} Y_{n}(0)\right| X_{s}\right\rangle\left\langle X_{s}\left|Y_{n}^{\dagger} \bar{Y}_{\bar{n}}^{\dagger}(0)\right| 0\right\rangle,
\end{aligned}
$$

where we have dropped the "res." label on the sums, because all restrictions are now explicitly implemented.

To see that the hemisphere definition of $s_{t}$ and $s_{\bar{t}}$ can be used to select dijetlike events, we can check that Eq. (62) plus the $\delta$ functions in Eq. (78) are sufficient to constrain the thrust to the dijet region. At leading order the total thrust of an event is given by

$$
Q T=\left|p_{n}^{z}\right|+\left|p_{\bar{n}}^{z}\right|+\left|k_{s}^{a z}\right|+\left|k_{s}^{b z}\right|,
$$

where $2\left|p_{n}^{z}\right|=Q+k_{n}^{-}-k_{n}^{+}$and $2\left|p_{\bar{n}}^{z}\right|=Q+k_{\bar{n}}^{+}-k_{\bar{n}}^{-}$. So the shifted thrust defined in Eq. (60) is

$$
\begin{aligned}
\tau= & -\frac{2 m^{2}}{Q^{2}}+\frac{1}{2 Q}\left[k_{n}^{+}-k_{n}^{-}+k_{\bar{n}}^{-}-k_{\bar{n}}^{+}+k_{s}^{a+}\right. \\
& \left.-k_{s}^{a-}+k_{s}^{b-}-k_{s}^{b+}\right] \\
= & -\frac{2 m^{2}}{Q^{2}}+\frac{1}{Q}\left[k_{n}^{+}+k_{s}^{a+}+k_{\bar{n}}^{-}+k_{s}^{b-}\right] \\
= & \frac{s_{t}+s_{\bar{t}}}{Q^{2}} .
\end{aligned}
$$

To obtain the second line we used the separate conservation of the + and - momentum components to eliminate $k_{n}^{-}+$ $k_{s}^{b-}$ and $k_{\bar{n}}^{+}+k_{s}^{a+}$. For the last line we used the $\delta$ functions 
in Eq. (76) to get $s_{t}$ and $s_{\bar{t}}$. Thus, the restriction to small hemisphere invariant masses $s_{t, \bar{t}}$ automatically gives small $\tau$ and restricts the events to the dijet region. The presence of a third hard jet takes us away from the dijet region and directly shows up by a substantial positive shift of $s_{t}+s_{\bar{t}}$ away from the peak region.

Next we simplify the form of the cross section by defining the massive collinear jet functions $\bar{h}$ as

$$
\begin{aligned}
\sum_{X_{n}} \operatorname{tr}\left\langle 0\left|\hat{\bar{h}} \chi_{n}(x)\right| X_{n}\right\rangle\left\langle X_{n}\left|\bar{\chi}_{n, Q}(0)\right| 0\right\rangle & =Q \int \frac{d^{4} r_{n}}{(2 \pi)^{3}} e^{-i r_{n} \cdot x} J_{n}\left(Q r_{n}^{+}-m^{2}, m\right) \\
& =Q \delta\left(x^{+}\right) \delta^{2}\left(x_{\perp}\right) \int d r_{n}^{+} e^{-(i / 2) r_{n}^{+} x^{-}} J_{n}\left(Q r_{n}^{+}-m^{2}, m\right), \\
\sum_{X_{\bar{n}}} \operatorname{tr}\left\langle 0\left|\bar{\chi}_{\bar{n}}(y)\right| X_{\bar{n}}\right\rangle\left\langle X_{\bar{n}}\left|\hat{\not} \chi_{\bar{n},-Q}(0)\right| 0\right\rangle & =Q \int \frac{d^{4} r_{\bar{n}}}{(2 \pi)^{3}} e^{-i r_{\bar{n}} \cdot y} J_{\bar{n}}\left(Q r_{\bar{n}}^{-}-m^{2}, m\right) \\
& =Q \delta\left(y^{-}\right) \delta^{2}\left(y_{\perp}\right) \int d r_{\bar{n}}^{-} e^{-(i / 2) r_{\bar{n}}^{-} y^{+}} J_{\bar{n}}\left(Q r_{\bar{n}}^{-}-m^{2}, m\right) .
\end{aligned}
$$

Here $m$ is the pole mass just as in Eq. (75) and we do not display the $\mu$ dependence. Note that the subscript $Q$ on the left-hand side does not change the mass dimension of a $\chi$-field away from $3 / 2$, since $\delta_{O, \overline{\mathcal{P}}}$ is dimensionless. We remind the reader that $\not{h}=\not h /\left(4 N_{c}\right), \not{h}=\bar{h} /\left(4 N_{c}\right)$ and that tr is a trace over both color and spin indices. The arguments of the jet functions, $J_{n}$ and $J_{\bar{n}}$, in Eq. (81) are just the offshellness of the jets, $p_{n}^{2}-m^{2}$ and $p_{\bar{n}}^{2}-m^{2}$, respectively, but given in expanded form. Here the labels $Q$ on the $\bar{\chi}_{n}$ and $\chi_{\bar{n}}$ fields ensures that there is only a contribution from the required "quark" and "antiquark" cut since $Q>0$. To see this recall that the sign of the label $p$ on $\xi_{n, p}$ picks out the quark annihilation, or antiquark production part of the field [27]. We note that the sums over collinear states in the collinear jet functions are unrestricted since the restrictions are now implemented automatically through the amount the jet invariant mass differs from $m^{2}$. Thus, the jet functions can be written as the discontinuity of a forward scattering amplitude after summing over the collinear states:

$$
\begin{aligned}
& J_{n}\left(Q r_{n}^{+}-m^{2}, m\right)=\frac{-1}{2 \pi Q} \operatorname{Disc} \int d^{4} x e^{i r_{n} \cdot x}\left\langle 0\left|\mathrm{~T}\left\{\bar{\chi}_{n, Q}(0) \hat{\bar{h}} \chi_{n}(x)\right\}\right| 0\right\rangle, \\
& J_{\bar{n}}\left(Q r_{\bar{n}}^{-}-m^{2}, m\right)=\frac{1}{2 \pi Q} \operatorname{Disc} \int d^{4} x e^{i r_{\bar{n}} \cdot x}\left\langle 0\left|\mathrm{~T}\left\{\bar{\chi}_{\bar{n}}(x) \hat{h} \chi_{\bar{n},-Q}(0)\right\}\right| 0\right\rangle .
\end{aligned}
$$

The collinear fields in the SCET jet functions $J_{n}$ and $J_{\bar{n}}$ are defined with zero-bin subtractions [54], which avoids double counting with the soft function. Using Eq. (81) and performing all the remaining integrals in the cross section of Eq. (78) we arrive at the SCET result for the double differential hemisphere invariant mass cross section

$$
\frac{d^{2} \sigma}{d M_{t}^{2} d M_{\bar{t}}^{2}}=\sigma_{0} H_{Q}(Q, \mu) \mathcal{M}(m, \mu) \int_{-\infty}^{\infty} d \ell^{+} d \ell^{-} J_{n}\left(s_{t}-Q \ell^{+}, m, \mu\right) J_{\bar{n}}\left(s_{\bar{t}}-Q \ell^{-}, m, \mu\right) S_{\mathrm{hemi}}\left(\ell^{+}, \ell^{-}, \mu, m\right),
$$

where the hard function $H_{Q}(Q, \mu)=|C(Q, \mu)|^{2}$. Here the hemisphere soft function in SCET is defined by

$$
S_{\text {hemi }}\left(\ell^{+}, \ell^{-}, \mu, m\right)=\frac{1}{N_{c}} \sum_{X_{s}} \delta\left(\ell^{+}-k_{s}^{+a}\right) \delta\left(\ell^{-}-k_{s}^{-b}\right)\left\langle 0\left|\bar{Y}_{\bar{n}} Y_{n}(0)\right| X_{s}\right\rangle\left\langle X_{s}\left|Y_{n}^{\dagger} \bar{Y}_{\bar{n}}^{\dagger}(0)\right| 0\right\rangle .
$$

At tree level for stable top quarks $H_{Q}=1, J_{n}\left(s_{t}\right)=\delta\left(s_{t}\right)$, $J_{\bar{n}}\left(s_{\bar{t}}\right)=\delta\left(s_{\bar{t}}\right)$, and $S_{\text {hemi }}\left(\ell^{+}, \ell^{-}\right)=\delta\left(\ell^{+}\right) \delta\left(\ell^{-}\right)$, and integrating Eq. (83) over $s_{t}$ and $s_{\bar{t}}$ gives the total tree-level Born cross section $\sigma_{0}$. This provides a check for the normalization of Eq. (83). The argument $m$ on the soft function in Eq. (84) and $\mathcal{M}(m, \mu)$ in Eq. (83) account for massive top quark bubbles that are perturbative and start at $\mathcal{O}\left(\alpha_{s}^{2}(m)\right)$ [71-73]. Note that Eq. (83) extends the SCET computation of the massless dijet cross section in Ref. [34,57] to all orders in perturbation theory for the jet functions.
In the factorization theorem in Eq. (83) the jet functions $J_{n}$ and $J_{\bar{n}}$ describe the dynamics of the top and antitop jets. In the next section we will see that these jet functions can be computed in perturbation theory and at the tree level are just Breit-Wigner distributions. The soft matrix elements $\left\langle 0\left|Y_{n}^{\dagger} Y_{\bar{n}}(0)\right| X_{s}\right\rangle\left\langle X_{s}\left|\tilde{Y}_{\bar{n}}^{\dagger} \tilde{Y}_{n}(0)\right| 0\right\rangle$, on the other hand, depends on the scale $\Lambda_{\mathrm{QCD}}$, and thus the soft function $S_{\text {hemi }}\left(\ell^{+}, \ell^{-}\right)$ is governed by nonperturbative QCD effects. The momentum variables $\ell^{ \pm}$represent the light-cone momentum of the soft particles in each of the two hemispheres, and $S_{\text {hemi }}\left(\ell^{+}, \ell^{-}\right)$describes the distribution of soft final-state 
radiation. Equation (83) already demonstrates that the invariant mass spectrum for unstable top quarks is not a Breit-Wigner function even at tree level because the convolution with the soft function $S_{\text {hemi }}$ modifies the observed distribution. The effects of the convolution on the observable invariant mass distribution are discussed in Sec. IV.

To sum large logs in Eq. (83) the SCET production current can be run from $\mu=Q$ down to $\mu=m$, which then characterizes the typical virtuality of the collinear degrees of freedom in massive SCET. In the process, large logarithms of $Q / m$ are summed into the hard function $H_{Q}(Q, \mu)$. In the next section we integrate out the scale $m$ and match these SCET jet functions onto bHQET jet functions.

\section{F. Factorization of jet mass effects in HQET}

The main result of the last subsection is the factorization of the scales $Q$ and $m$ in the differential cross section of Eq. (83). In this section we further factorize the scale $m$ from the low-energy scales $\Gamma, \hat{s}$, and $\Delta$. This will allow us to sum large logs of $\Gamma / m$ and $\hat{s}_{t, \bar{t}} / m$ in the jet functions, and lower the scale of the soft functions to $\Delta$. This step is also important for treating the width effects. As explained earlier, one can formulate width effects in a gauge invariant way with a natural power counting in HQET, whereas doing so in a relativistic theory such as SCET is notoriously difficult.

To perform the scale separation and sum the logarithms requires us to match and run below the scale $\mu=m$. This can be done in a standard way, by matching and running of the bHQET current in Eq. (37), as we described in Sec. II B. However, due to the factorization properties of SCET which leads to a decoupling of the $n$-collinear, $\bar{n}$-collinear, and soft sectors, the matching and running below the scale $\mu=m$ can also be done independently for $J_{n}, J_{\bar{n}}$, and $S$. In the following we explain this second method.

As discussed in Sec. II B the soft function above and below the scale $m$ is identical, except for certain vacuum polarization effects from graphs with top quark bubbles that only exist in SCET. For the soft function in bHQET we have

$$
\begin{aligned}
S_{\text {hemi }}\left(\ell^{+}, \ell^{-}, \mu\right)= & \frac{1}{N_{c}} \sum_{X_{s}} \delta\left(\ell^{+}-k_{s}^{+a}\right) \delta\left(\ell^{-}-k_{s}^{-b}\right) \\
& \times\left\langle 0\left|\bar{Y}_{\bar{n}} Y_{n}(0)\right| X_{s}\right\rangle\left\langle X_{s}\left|Y_{n}^{\dagger} \bar{Y}_{\bar{n}}^{\dagger}(0)\right| 0\right\rangle,
\end{aligned}
$$

where there is no $m$ dependence. The matching condition between the soft functions in the two theories is

$$
S_{\text {hemi }}\left(\ell^{+}, \ell^{-}, \mu, m\right)=T_{0}(m, \mu) S_{\text {hemi }}\left(\ell^{+}, \ell^{-}, \mu\right),
$$

where $T_{0}(m, \mu)$ is a Wilson coefficient. Large logarithms in the soft function can be summed by computing the

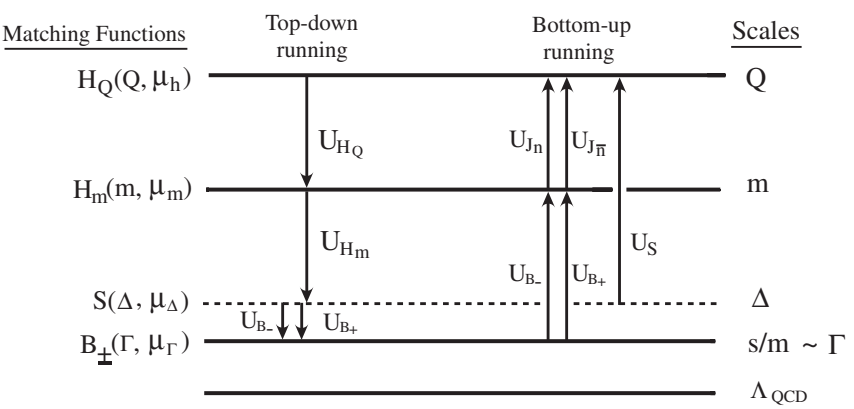

FIG. 5. Scales and functions appearing in the formula for the invariant mass distribution. The result is determined by matching at the physical scales and running to sum large logs as shown. We show both the top-down and bottom-up approach to the running. The evolution for $U_{H}$ and $U_{C}$ is local, while all other evolution functions involve convolutions. Note that the evolution functions obey $U_{H}=U_{J_{-}} \otimes U_{J_{+}} \otimes U_{S}$ and $U_{C}=U_{B_{-}} \otimes U_{B_{+}} \otimes$ $U_{S}$ where $\otimes$ indicates convolutions.

anomalous dimension of the soft function and using $\mathrm{RG}$ evolution to run between $\Delta$ and $m$ and between $m$ and $Q$ as illustrated by the line labeled $U_{S}$ in Fig. 5 .

For the SCET collinear degrees of freedom the power counting for the virtuality is $p_{c}^{2} \sim m^{2}$. Thus, $J_{n}$ and $J_{\bar{n}}$ describe the physics of jets with an invariant mass up to $M^{2} \sim \mu^{2} \sim m^{2}$. However, the restriction of being in the peak region means that $M^{2}-m^{2} \ll m^{2}$. This disparity gives rise to the large logarithms in the collinear jet functions. Intuitively, this can also be understood by noting that if one starts out with a top quark that is close to its mass shell, a typical collinear SCET gluon will knock the top far off shell so that $p_{c}^{2}-m^{2} \sim m^{2}$. By restricting the jet functions to $p_{c}^{2}-m^{2} \ll m^{2}$ we forbid such real radiation contributions, but not virtual contributions. The latter must be integrated out explicitly by switching to the description of the jet functions in the boosted unstable HQET theories discussed in Sec. II B. In these HQETs the only fluctuations are due to low energy ultracollinear gluons that preserve the condition $M^{2}-m^{2} \ll m^{2}$.

To determine the definitions of the bHQET jet functions we follow the same procedure as for the bHQET current in Eq. (37), namely, boost the SCET jet function in Eq. (82) to the heavy quark rest frame, giving $\bar{\psi}(x) W(x) W(0) \psi(0)$, then match onto HQET $\psi(x) \rightarrow h_{v}(x)$. We then boost back to the moving frame where $v \rightarrow v_{ \pm}$. The spin structure can also be simplified to give

$$
\begin{aligned}
\frac{1}{Q} \bar{\chi}_{n, Q} \hat{\bar{h}} \chi_{n} & \rightarrow \frac{1}{Q} \bar{h}_{v_{+}} \hat{\bar{h}} h_{v_{+}}=\frac{v_{+} \cdot \bar{n}}{4 N_{c} Q} \bar{h}_{v_{+}} h_{v_{+}} \\
& =\frac{1}{4 N_{c} m} \bar{h}_{v_{+}} h_{v_{+}} .
\end{aligned}
$$

Thus the bHQET jet functions are defined as 


$$
\begin{aligned}
& B_{+}\left(2 v_{+} \cdot k\right)=\frac{-1}{8 \pi N_{c} m} \operatorname{Disc} \int d^{4} x e^{i k \cdot x}\left\langle 0\left|\mathrm{~T}\left\{\bar{h}_{v_{+}}(0) W_{n}(0) W_{n}^{\dagger}(x) h_{v_{+}}(x)\right\}\right| 0\right\rangle, \\
& B_{-}\left(2 v_{-} \cdot k\right)=\frac{1}{8 \pi N_{c} m} \operatorname{Disc} \int d^{4} x e^{i k \cdot x}\left\langle 0\left|\mathrm{~T}\left\{\bar{h}_{v_{-}}(x) W_{\bar{n}}(x) W_{\bar{n}}^{\dagger}(0) h_{v_{-}}(0)\right\}\right| 0\right\rangle .
\end{aligned}
$$

These bHQET jet functions can be calculated using the usual Feynman rules of HQET except that the gluons have ucollinear scaling as in Eq. (34). The $W$-Wilson lines in $B_{ \pm}$ also contain these boosted gluons. Since $p_{n}^{2}-m^{2}=$ $2 m v_{+} \cdot k$ and $p_{\bar{n}}^{2}-m^{2}=2 m v_{-} \cdot k$, we can identify the arguments of the bHQET jet functions as

$$
2 v_{+} \cdot k=\frac{s_{t}}{m}=\hat{s}_{t}, \quad 2 v_{-} \cdot k=\frac{s_{\bar{t}}}{m}=\hat{s}_{\bar{t}} .
$$

In the factorization theorem these arguments are shifted by the soft gluon momenta as shown in Eq. (94) below. Recall that the fields $h_{v_{+}}$and $h_{v_{-}}$are defined with zero-bin subtractions on their ultracollinear momenta. For Eq. (88) these subtractions can be thought of as being inherited from the SCET fields in the matching. They remove the light-cone singularities $n \cdot k \rightarrow 0$ and $\bar{n} \cdot k \rightarrow 0$ in $B_{+}$and $B_{-}$, respectively, and are important to ensure that the width $\Gamma$ is sufficient to make $B_{ \pm}$infrared finite.

In general the matching of the jet functions in SCET onto those in bHQET could take the form

$$
J_{n, \bar{n}}(m \hat{s}, m, \Gamma, \mu)=\int_{-\infty}^{\infty} d \hat{s}^{\prime} T_{ \pm}\left(\hat{s}, \hat{s}^{\prime}, m, \mu\right) B_{ \pm}\left(\hat{s}^{\prime}, \Gamma, \mu\right),
$$

where the convolution takes into account the fact that depending on the definition, the observable $\hat{s}$ could be sensitive to scales of $\mathcal{O}(m)$ and $\mathcal{O}(\Gamma)$. In such a case, since $\hat{s}^{\prime}$ does not know about the scale $m$, it cannot be identical to $\hat{s}$. The convolution with $T_{ \pm}\left(\hat{s}, \hat{s}^{\prime}, m, \mu\right)$ then compensates for this difference. In our case (and most reasonable cases) the definition of the invariant mass is not sensitive to $m$, so we have $T_{ \pm}\left(\hat{s}, \hat{s}^{\prime}, m, \mu\right)=\delta\left(\hat{s}-\hat{s}^{\prime}\right) T_{ \pm}(m, \mu)$ and the matching equations are simply

$$
\begin{aligned}
& J_{n}\left(m \hat{s}, m, \Gamma, \mu_{m}\right)=T_{+}\left(m, \mu_{m}\right) B_{+}\left(\hat{s}, \Gamma, \mu_{m}\right), \\
& J_{\bar{n}}\left(m \hat{s}, m, \Gamma, \mu_{m}\right)=T_{-}\left(m, \mu_{m}\right) B_{-}\left(\hat{s}, \Gamma, \mu_{m}\right) .
\end{aligned}
$$

Since there are no mass modes in bHQET the function $\mathcal{M}$ also appears as part of the Wilson coefficient. From this we define a hard coefficient that contains the mass corrections as $^{4}$

$H_{m}\left(m, \mu_{m}\right)=T_{+}\left(m, \mu_{m}\right) T_{-}\left(m, \mu_{m}\right) T_{0}\left(m, \mu_{m}\right) \mathcal{M}\left(m, \mu_{m}\right)$.

\footnotetext{
${ }^{4}$ In explicit computations scheme dependence may affect the manner in which the mass corrections are divided up between $T_{ \pm}, T_{0}$, and $\mathcal{M}$; however, this dependence will cancel out in the product that gives $H_{m}$.
}

By charge conjugation we know that the jet functions for the top and antitop have the same functional form, and that $T_{+}=T_{-}$. When we sum large logs into the coefficient $H_{m}$ it develops an additional dependence on $Q / m$ through its anomalous dimension which depends on $v_{+} \cdot \bar{n}=$ $v_{-} \cdot n=Q / m$. Note that in principle $H_{m}(m, \mu)$ and the factors in Eq. (92) can also have $Q / m$ dependence at nextto-next-to leading logarithmic order. Contributions related to the mass modes in Bhabbha scattering due to virtual QED electron loops corrections at the two-loop level have been determined in Ref. [74]. A treatment of mass mode type effects for massive gauge boson effects in electroweak corrections for large energies has been given in Ref. [75].

Since the functions $T_{ \pm}$are independent of the top width $\Gamma$, we are free to set $\Gamma=0$ (i.e., use stable top quarks) for the matching calculations at any order in perturbation theory. At tree level we need to compute the discontinuity of the graphs in Fig. 6 which have a trace over spin and color indices. For $\Gamma=0$ this gives

$$
\begin{aligned}
B_{+}^{\text {tree }}(\hat{s}, \Gamma=0) & =\frac{-1}{8 \pi N_{c} m}\left(-2 N_{c}\right) \operatorname{Disc}\left(\frac{i}{v_{+} \cdot k+i 0}\right) \\
& =\frac{1}{4 \pi m} \operatorname{Im}\left(\frac{-2}{v_{+} \cdot k+i 0}\right) \\
& =\frac{1}{m} \delta\left(2 v_{+} \cdot k\right)=\frac{1}{m} \delta(\hat{s})=\delta(s),
\end{aligned}
$$

which is identical to the result for the corresponding SCET jet function, so at tree level $T_{+}=T_{-}=1$.

Plugging Eq. (91) into Eq. (83), and incorporating renormalization group evolution, the form for the differential cross section is

$$
\begin{aligned}
\left(\frac{d^{2} \sigma}{d M_{t}^{2} d M_{\bar{t}}^{2}}\right)_{\mathrm{hemi}}= & \sigma_{0} H_{Q}\left(Q, \mu_{m}\right) H_{m}\left(m, \frac{Q}{m}, \mu_{m}, \mu\right) \\
& \times \int_{-\infty}^{\infty} d \ell^{+} d \ell^{-} B_{+}\left(\hat{s}_{t}-\frac{Q \ell^{+}}{m}, \Gamma, \mu\right) \\
& \times B_{-}\left(\hat{s}_{\bar{t}}-\frac{Q \ell^{-}}{m}, \Gamma, \mu\right) S_{\mathrm{hemi}}\left(\ell^{+}, \ell^{-}, \mu\right) .
\end{aligned}
$$

Equation (94) is our final result in terms of the pole mass $m$. The analogous result for a short-distance mass is given a)

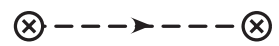

b)

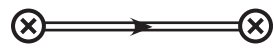

FIG. 6. Tree-level top quark jet functions in (a) SCET and (b) bHQET. 
in the next section. Here $H_{m}\left(m, Q / m, \mu_{m}, \mu\right)$ is the hard coefficient $H_{m}\left(m, \mu_{m}\right)$ run down from $\mu_{m}$ to $\mu$, and we still have $H_{Q}\left(Q, \mu_{m}\right)=\left|C\left(Q, \mu_{m}\right)\right|^{2}$, and the soft function with Wilson lines evaluated at $x=0$,

$$
\begin{aligned}
S_{\text {hemi }}\left(\ell^{+}, \ell^{-}, \mu\right)= & \frac{1}{N_{c}} \sum_{X_{s}} \delta\left(\ell^{+}-k_{s}^{+a}\right) \delta\left(\ell^{-}-k_{s}^{-b}\right) \\
& \times\left\langle 0\left|\left(\bar{Y}_{\bar{n}}\right) c^{\prime}\left(Y_{n}\right)^{c b^{\prime}}\right| X_{s}\right\rangle \\
& \times\left\langle X_{s}\left|\left(Y_{n}^{\dagger}\right)^{b^{\prime} c^{\prime}}\left(\bar{Y}_{\bar{n}}^{\dagger}\right)^{a^{\prime} c^{\prime}}\right| 0\right\rangle .
\end{aligned}
$$

For completeness we wrote out the color indices from Eq. (52). It is interesting to note that in the result in Eq. (94) the final matrix elements only involve Wilson lines (since the coupling of gluons to a heavy quark field $h_{v_{+}}$in $B_{+}$is the same as to a Wilson line $W_{v_{+}}$).

To conclude this section we finally repeat the computation of the tree level bHQET jet functions, but now for the realistic case with $\Gamma \neq 0$ in the HQET propagators. The computation is done at a scale $\mu \gtrsim \Gamma$, but the $\mu$ dependence does not show up at tree level. Figure 6(b) gives

$$
\begin{aligned}
B_{ \pm}^{\text {tree }}(\hat{s}, \Gamma) & =\frac{-1}{8 \pi N_{c} m}\left(-2 N_{c}\right) \operatorname{Disc}\left(\frac{i}{v_{ \pm} \cdot k+i \Gamma / 2}\right) \\
& =\frac{1}{4 \pi m} \operatorname{Im}\left(\frac{-2}{v_{ \pm} \cdot k+i \Gamma / 2}\right)=\frac{1}{\pi m} \frac{\Gamma}{\hat{s}^{2}+\Gamma^{2}} .
\end{aligned}
$$

Thus we see that $B_{ \pm}(\hat{s})$ are equal to Breit-Wigner functions at lowest order in $\alpha_{s}$. At higher orders in perturbation theory the width will cut off the IR divergences that would otherwise occur at $\hat{s}=0$. The functions $B_{ \pm}$at the scale $\mu$ can therefore be computed perturbatively to any desired order in $\alpha_{s}$. In general the perturbative "matching" corrections will lead to distortions of the tree-level BreitWigner distributions shown in Eq. (96), as does the potential separate running between $\mu_{\Delta}$ and $\mu_{\Gamma}$ discussed below in Sec. III H and shown in Fig. 5.

\section{G. A short-distance top mass for jets}

The derivation of the factorization formulas (94) in the previous section was given in the pole mass scheme, ${ }^{5} m_{\text {pole }}$. It is, however, well known that the pole mass definition leads to an artificially enhanced sensitivity to small momenta in Feynman diagrams (see Ref. [76] for a review) and, as a consequence, to artificially large perturbative corrections. This behavior is particularly important for observables that have a strong dependence on the heavy quark mass [15,58-61]. From a nonperturbative point of view, this feature is related to an intrinsic ambiguity in the heavy quark pole mass parameter of order the hadronization scale $\Lambda_{\mathrm{QCD}}$ and is sometimes referred to as the

\footnotetext{
${ }^{5}$ In Eq. (94) we used $m$ for the pole mass, but in this section we write $m_{\text {pole }}$, and reserve " $m$ " for a generic mass scheme.
}

$\mathcal{O}\left(\Lambda_{\mathrm{QCD}}\right)$-renormalon problem of the pole mass. Heavy quark mass definitions that do not have such an $\mathcal{O}\left(\Lambda_{\mathrm{QCD}}\right)$ ambiguity are called short-distance mass schemes. ${ }^{6}$ In the factorization formulas in Eq. (94), the top mass appears in the hard function $H_{m}$ and in the two jet functions $B_{+}\left(\hat{s}_{t}\right)$ and $B_{-}\left(\hat{s}_{\bar{t}}\right)$. The most important sensitivity to the top-mass scheme is in $\hat{s}_{t}=\left(M_{t}^{2}-m^{2}\right) / m$ and $\hat{s}_{\bar{t}}=\left(M_{\bar{t}}^{2}-m^{2}\right) / m$, where $M_{t}^{2}$ and $M_{\bar{t}}^{2}$ are scheme independent observables.

A specific short-distance top quark mass scheme " $m$ " can be defined by a finite residual mass term $\delta m \neq 0$, as

$$
m_{\text {pole }}=m+\delta m,
$$

where $\delta m$ starts at $\mathcal{O}\left(\alpha_{s}\right)$ or higher, and must be strictly expanded perturbatively to the same order as other $\mathcal{O}\left(\alpha_{s}\right)$ corrections. (This strict expansion does not apply to powers of $\alpha_{s}$ times $\operatorname{logs}$ that are summed up by renormalization group improved perturbation theory.) Let $B_{+}(\hat{s}, \mu, \delta m)$ denote the jet function in the short-distance mass scheme specified by $\delta m$. We can calculate $B_{+}(\hat{s}, \mu, \delta m)$ in two equivalent ways: (i) Use the pole mass scheme initially by setting $\delta m=0$ in Eq. (33). In this case the mass dependence appears in $\hat{s}_{\text {pole }}=\left(M^{2}-m_{\text {pole }}^{2}\right) / m_{\text {pole }}$ in $B_{+}$and we change the scheme with Eq. (97). Alternatively, (ii) treat $\delta m \neq 0$ in Eq. (33) as a vertex in Feynman diagrams, and take $\hat{s}$ to be defined in the short-distance mass scheme right from the start, so $\hat{s}=\left(M^{2}-m^{2}\right) / m$.

As discussed in Sec. II B, it is necessary that the residual mass term is consistent with the bHQET power counting, i.e.

$$
\delta m \sim \hat{s}_{t} \sim \hat{s}_{\bar{t}} \sim \Gamma .
$$

Equation (98) restricts us to a suitable class of shortdistance mass schemes for jets. In any short-distance mass scheme which violates Eq. (98) the EFT expansion breaks down, and thus the notion of a top quark BreitWigner distribution becomes invalid. The most prominent example for an excluded short-distance mass scheme is the $\overline{\mathrm{MS}}$ mass scheme, $\bar{m}$, for which $m_{\text {pole }}-\bar{m}=\delta \bar{m}$. Here $\delta \bar{m} \simeq 8 \mathrm{GeV} \gg \Gamma$, or parametrically $\delta \bar{m} \sim \alpha_{s} \bar{m} \gg \Gamma$. Using Eq. (96) and converting to the $\overline{\mathrm{MS}}$ scheme with the $\mathcal{O}\left(\alpha_{s}\right)$ residual mass term we have

$$
B_{+}(\hat{s}, \mu, \delta \bar{m})=\frac{1}{\pi \bar{m}}\left\{\frac{\Gamma}{\left[\frac{\left(M_{t}^{2}-\bar{m}^{2}\right)^{2}}{\bar{m}^{2}}+\Gamma^{2}\right]}+\frac{(4 \hat{s} \Gamma) \delta \bar{m}}{\left[\frac{\left(M_{t}^{2}-\bar{m}^{2}\right)^{2}}{\bar{m}^{2}}+\Gamma^{2}\right]^{2}}\right\} .
$$

Here the first term is $\sim 1 /(\bar{m} \Gamma)$ and is swamped by the second term $\sim \alpha_{s} / \Gamma^{2}$, which is supposed to be a perturbative correction. This means that it is not the $\overline{\mathrm{MS}}$ mass that is

\footnotetext{
${ }^{6}$ In practice, determining the pole mass from the analysis of experimental data leads to values that depend strongly on the order of perturbation theory that has been employed for the theoretical predictions. This makes the treatment of theoretical errors difficult.
} 
ever directly measured from any reconstruction mass measurement that uses a top Breit-Wigner at some level of the analysis. We stress that this statement applies to any topmass determination that relies on the reconstruction of the peak position of an invariant mass distribution.

To define a short-distance scheme for jet reconstruction measurements, $m_{J}$, we choose the residual mass term $\delta m_{J}$ such that, order-by-order, the jet functions $B_{ \pm}$have their maximum at $\hat{s}_{t}=\hat{s}_{\bar{t}}=0$, where $B_{+}(\hat{s})$ is the gauge invariant function defined in Eq. (88). So order-by-order in perturbation theory the definition is given by the solution to

$$
\left.\frac{d B_{+}\left(\hat{s}, \mu, \delta m_{J}\right)}{d \hat{s}}\right|_{\hat{s}=0}=0 .
$$

We call this mass definition the top quark jet mass, $m_{J}(\mu)=m_{\text {pole }}-\delta m_{J}$. Since the bHQET jet functions have a nonvanishing anomalous dimension, the top jetmass depends on the renormalization scale $\mu$, at which the jet functions are computed perturbatively. Thus the jet mass is a running mass, similar to the $\overline{\mathrm{MS}}$ mass, and different choices for $\mu \gtrsim \Gamma$ can in principle be made.

For simplicity we will use the notation $\tilde{B}_{ \pm}(\hat{s}, \mu)$ for the bHQET jet functions in the jet-mass scheme. At next-toleading order in $\alpha_{s}$,

$$
\tilde{B}_{ \pm}(\hat{s}, \mu)=B_{ \pm}(\hat{s}, \mu)+\frac{1}{\pi m_{J}} \frac{(4 \hat{s} \Gamma) \delta m_{J}}{\left(\hat{s}^{2}+\Gamma^{2}\right)^{2}},
$$

where $m_{J}=m_{J}(\mu)$ and $B_{+}$is the pole mass jet function to $\mathcal{O}\left(\alpha_{s}\right)$. Here we dropped all corrections that are power suppressed by $\Gamma / \mathrm{m}$. The one-loop relation between the pole and jet mass is [66]

$$
m_{J}(\mu)=m_{\text {pole }}-\Gamma \frac{\alpha_{s}(\mu)}{3}\left[\ln \left(\frac{\mu}{\Gamma}\right)+\frac{3}{2}\right] .
$$

For $\mu=\Gamma$ we have $\delta m_{J} \simeq 0.26 \mathrm{GeV}$, so the jet mass is quite close to the one-loop pole mass. Equation (102) also shows that the jet mass is substantially different from the short-distance masses that are employed for $t \bar{t}$-threshold analyses [15], where $\delta m \sim \alpha_{s}^{2} m \sim 2 \mathrm{GeV}$ is of order the binding energy of the $t \bar{t}$ quasibound state. Nevertheless, in some of the threshold mass schemes $[59,60] \delta m$ is proportional to a cutoff scale that could in principle be adapted such that they are numerically close to the jet mass we are proposing. A detailed discussion on the impact of switching from the pole to the jet-mass scheme at the one-loop level and at higher orders will be given in Refs. [66,77], respectively. We remark that many other schemes satisfying Eq. (98) can in principle be defined, but the existence of one such scheme suffices. However, for any suitable shortdistance mass scheme the renormalization scale in $\alpha_{s}$ contained in $\delta m$ has to be equal to the scale $\mu$ used for the computation of the bHQET jet functions.

The other function that must be modified in the factorization theorem is $H_{m}\left(m, Q / m, \mu_{m}, \mu_{\Delta}\right)$. However, this function only depends logarithmically on $m$, and

$$
\ln \left(\frac{m_{\text {pole }}}{\mu}\right)=\ln \left(\frac{m_{J}}{\mu}\right)+\mathcal{O}\left(\frac{\alpha_{s} \Gamma}{m_{J}}\right) \text {. }
$$

So dropping these perturbatively suppressed power corrections we can simply replace $m \rightarrow m_{J}$ in $H_{m}$. We note that any $\mu$ dependence from $m_{J}(\mu)$ in $H_{m}$ is also power suppressed.

Thus our final result for the cross section in terms of the short-distance jet mass is

$$
\begin{aligned}
\left(\frac{d^{2} \sigma}{d M_{t}^{2} d M_{\bar{t}}^{2}}\right)_{\mathrm{hemi}}= & \sigma_{0} H_{Q}\left(Q, \mu_{m}\right) H_{m}\left(m_{J}, \frac{Q}{m_{J}}, \mu_{m}, \mu\right) \\
& \times \int_{-\infty}^{\infty} d \ell^{+} d \ell^{-} \tilde{B}_{+}\left(\hat{s}_{t}-\frac{Q \ell^{+}}{m_{J}}, \Gamma, \mu\right) \\
& \times \tilde{B}_{-}\left(\hat{s}_{\bar{t}}-\frac{Q \ell^{-}}{m_{J}}, \Gamma, \mu\right) S_{\mathrm{hemi}}\left(\ell^{+}, \ell^{-}, \mu\right),
\end{aligned}
$$

where the running jet mass $m_{J}=m_{J}(\mu)$.

\section{H. Renormalization-group evolution}

In order to explain the $\mu$ dependence of the factorization theorem in Eq. (104) we give a brief discussion of the renormalization group evolution. A more detailed discussion is given in Ref. [66]. Equation (104) depends on two renormalization scales, $\mu_{m}$ and $\mu$. The matching scale $\mu_{m} \sim m$ was the endpoint of the evolution of the hard function $H_{Q}\left(Q, \mu_{m}\right)$. From the matching at $m$ we get the dependence on $\mu_{m}$ in $H_{m}$, and from running below $m$ we get an additional dependence on $\mu$ as well as $Q / m$ (which is discussed in more detail in Ref. [66] and signifies the presence of a cusp term in the anomalous dimension, see Ref. [78]). The $\mu$ dependence in $H_{m}$ cancels against the $\mu$ dependence in the bHQET jet functions and the soft function.

To sum the remaining large logarithms we have in principle two choices. We can either run the Wilson coefficient $H_{m}$, or we can run the individual functions $\tilde{B}_{ \pm}$and $S$. The first option essentially corresponds to running the bHQET top pair production current of Eq. (37), and we will call this method top-down. The relation

$$
H_{m}\left(m, \frac{Q}{m}, \mu_{m}, \mu\right)=H_{m}\left(m, \mu_{m}\right) U_{H_{m}}\left(\mu_{m}, \mu, \frac{Q}{m}\right)
$$

defines the corresponding evolution factor $U_{H_{m}}$ that is shown in Fig. 5. The second option means running the jet functions $\tilde{B}_{ \pm}$and the soft function $S_{\text {hemi }}$ independently with the evolution factors $U_{B_{ \pm}}\left(\mu, \mu_{m}\right)$ and $U_{S}\left(\mu, \mu_{m}\right)$, respectively, and is also illustrated in Fig. 5. This running involves convolutions, such as 


$$
\begin{aligned}
\mu \frac{d}{d \mu} \tilde{B}_{+}(\hat{s}, \mu) & =\int d \hat{s}^{\prime} \gamma_{B_{+}}\left(\hat{s}-\hat{s}^{\prime}\right) \tilde{B}_{+}\left(\hat{s}^{\prime}, \mu\right), \\
\tilde{B}_{+}\left(\hat{s}, \mu_{m}\right) & =\int d \hat{s}^{\prime} U_{B_{+}}\left(\hat{s}-\hat{s}^{\prime}, \mu_{m}, \mu\right) \tilde{B}_{+}\left(\hat{s}^{\prime}, \mu\right),
\end{aligned}
$$

and analogously for $\tilde{B}_{-}$and $S_{\text {hemi }}$. Since this method for the running usually involves taking the functions $B_{ \pm}$and $S_{\text {hemi }}$ as an input at the low scale (to avoid the appearance of large logs) we will call this option bottom-up. Because the running of $H_{m}$ is local (i.e. has no convolution), this RG evolution only affects the normalization of the cross section and does not change the dependence on $s_{t}$ and $s_{\bar{t}}$ in a nontrivial way. This is more difficult to discern from the bottom-up running, but when the convolutions for $B_{ \pm}$and $S$ are combined they must become local. These cancellations are discussed in detail in Ref. [66] where also the full leading $\log$ evolution is derived.

Generically, we may wish to run the soft function and jet function to slightly different low energy scales. Lets examine the case shown in Fig. 5 where we run the soft function to $\mu_{\Delta}$ but run the bHQET jet functions to a slightly lower scale $\mu_{\Gamma}$. (The opposite case could of course also be realized.) In this case the running is local up to the scale $\mu_{\Delta}$, and below this scale we have convolution running for $B_{ \pm}$. Using Eq. (106) the factorization formula for split low energy renormalization scales is

$$
\begin{aligned}
\frac{d^{2} \sigma}{d M_{t}^{2} d M_{\bar{t}}^{2}}= & \sigma_{0} H_{Q}\left(Q, \mu_{m}\right) H_{m}\left(m_{J}, \frac{Q}{m_{J}}, \mu_{m}, \mu_{\Delta}\right) \int_{-\infty}^{\infty} d \hat{s}_{t}^{\prime} d \hat{s}_{\bar{t}}^{\prime} U_{B_{+}}\left(\hat{s}_{t}-\hat{s}_{t}^{\prime}, \mu_{\Delta}, \mu_{\Gamma}\right) U_{B_{-}}\left(\hat{s}_{\bar{t}}-\hat{s}_{\bar{t}}^{\prime}, \mu_{\Delta}, \mu_{\Gamma}\right) \\
& \times \int_{-\infty}^{\infty} d \ell^{+} d \ell^{-} S_{\text {hemi }}\left(\ell^{+}, \ell^{-}, \mu_{\Delta}\right) \tilde{B}_{+}\left(\hat{s}_{t}^{\prime}-\frac{Q \ell^{+}}{m_{J}}, \Gamma, \mu_{\Gamma}\right) \tilde{B}_{-}\left(\hat{s}_{\bar{t}}^{\prime}-\frac{Q \ell^{-}}{m_{J}}, \Gamma, \mu_{\Gamma}\right),
\end{aligned}
$$

where parametrically $\mu_{\Delta} \sim \mu_{\Gamma}$ and here we take $m_{J}=$ $m_{J}\left(\mu_{\Gamma}\right)$. In this paper we will use common low energy scales for our numerical analysis, as shown in Eq. (104), and leave the discussion of the more general case in Eq. (107) to Ref. [66].

\section{Thrust and other event-shape variables}

Starting from the two-dimensional distribution, $d^{2} \sigma / d M_{t}^{2} d M_{\bar{t}}^{2}$ in Eq. (104) it is straightforward to derive results for other event-shape variables. For example, for the thrust $T$ defined in Eq. (59), we have $1-T=\left(M_{t}^{2}+\right.$ $\left.M_{\bar{t}}^{2}\right) / Q^{2}$ which follows using Eq. (80) with Eqs. (1) and (60). Inserting the identity

$$
1=\int d T \delta\left(1-T-\frac{M_{t}^{2}+M_{\bar{t}}^{2}}{Q^{2}}\right)
$$

into Eq. (104) and integrating over $M_{t}^{2}$ and $M_{\bar{t}}^{2}$ we find

$$
\begin{aligned}
\frac{d \sigma}{d T}= & \sigma_{0}^{H}(\mu) \int_{-\infty}^{\infty} d s_{t} d s_{\bar{t}} \tilde{B}_{+}\left(\frac{s_{t}}{m_{J}}, \Gamma, \mu\right) \tilde{B}_{-}\left(\frac{s_{\bar{t}}}{m_{J}}, \Gamma, \mu\right) \\
& \times S_{\text {thrust }}\left(1-T-\frac{\left(2 m_{J}^{2}+s_{t}+s_{\bar{t}}\right)}{Q^{2}}, \mu\right),
\end{aligned}
$$

where $\quad \sigma_{0}^{H}(\mu)=\sigma_{0} H_{Q}\left(Q, \mu_{m}\right) H_{m}\left(m_{J}, Q / m_{J}, \mu_{m}, \mu\right)$. Here the thrust soft function is simply a projection of the hemisphere soft function,

$$
\begin{aligned}
S_{\text {thrust }}(\tau, \mu)= & \int_{0}^{\infty} d \ell^{+} d \ell^{-} \delta\left(\tau-\frac{\left(\ell^{+}+\ell^{-}\right)}{Q}\right) \\
& \times S_{\text {hemi }}\left(\ell^{+}, \ell^{-}, \mu\right) \\
= & \frac{1}{N_{c}} \sum_{X_{s}} \delta\left(\tau-\frac{k_{s}^{+a}+k_{s}^{-b}}{Q}\right) \\
& \times\left\langle 0\left|\bar{Y}_{\bar{n}} Y_{n}(0)\right| X_{s}\right\rangle\left\langle X_{s}\left|Y_{n}^{\dagger} \bar{Y}_{\bar{n}}^{\dagger}(0)\right| 0\right\rangle .
\end{aligned}
$$

Another well-known distribution, which is also frequently analyzed for massless jets, is the heavy-jet mass. It can be defined by the dimensionless variable

$$
\rho=\frac{1}{Q^{2}} \max \left\{M_{t}^{2}, M_{\bar{t}}^{2}\right\} .
$$

Using the same steps as above for $\rho$, the factorization theorem for top initiated jets is

$$
\begin{aligned}
\frac{d \sigma}{d \rho}= & \sigma_{0}^{H}(\mu) \int_{-\infty}^{\infty} d s_{t} d s_{\bar{t}} \tilde{B}_{+}\left(\frac{s_{t}}{m_{J}}, \Gamma, \mu\right) \tilde{B}_{-}\left(\frac{s_{\bar{t}}}{m_{J}}, \Gamma, \mu\right) \\
& \times S_{\mathrm{HJM}}\left(\rho-\frac{m_{J}^{2}}{Q^{2}}, s_{t}, s_{\bar{t}}\right),
\end{aligned}
$$

where the relevant soft function is

$$
\begin{aligned}
S_{\mathrm{HJM}}\left(\rho, s_{t}, s_{\bar{t}}\right)= & \int_{0}^{\infty} d \ell^{+} d \ell^{-} \\
& \times \delta\left(\rho-\frac{1}{Q^{2}} \max \left\{Q \ell^{+}+s_{t}, Q \ell^{-}+s_{\bar{t}}\right\}\right) \\
& \times S_{\text {hemi }}\left(\ell^{+}, \ell^{-}, \mu\right) .
\end{aligned}
$$

Factorization theorems for other event shapes that are related to $d^{2} \sigma / d M_{t}^{2} d M_{\bar{t}}^{2}$ can be derived in an analogous manner. As should be obvious from the definitions of thrust and the heavy-jet mass distribution in Eqs. (109) and (112), 
these event-shape distributions are also characterized by a peak at shape parameter values that are sensitive to the short-distance top quark mass. It is therefore possible to use these event shapes to measure the top mass with a precision comparable to the invariant mass distribution discussed in the previous subsection. A brief numerical analysis of the thrust distribution is given in Sec. IVA.

\section{ANALYSIS OF THE INVARIANT MASS DISTRIBUTION}

\section{A. A simple leading order analysis}

The main result of this paper is the formula in Eq. (104) for the double invariant mass distribution with a shortdistance top quark mass suitable for measurements using jets. In this section we discuss the implications of Eq. (104) for top-mass measurements. For convenience we rewrite the cross section in terms of dimension one invariant mass variables

$$
\frac{d^{2} \sigma}{d M_{t} d M_{\bar{t}}}=\frac{4 M_{t} M_{\bar{t}} \sigma_{0}^{H}}{\left(m_{J} \Gamma\right)^{2}} F\left(M_{t}, M_{\bar{t}}, \mu\right),
$$

where $\sigma_{0}^{H}=\sigma_{0} H_{Q}\left(Q, \mu_{m}\right) H_{m}\left(m_{J}, Q / m_{J}, \mu_{m}, \mu\right)$ is the cross section normalization factor with radiative corrections, $Q$ is the c.m. energy, and we have defined a dimensionless function

$$
\begin{aligned}
F\left(M_{t}, M_{\bar{t}}, \mu\right)= & \left(m_{J} \Gamma\right)^{2} \int_{-\infty}^{\infty} d \ell^{+} d \ell^{-} \tilde{B}_{+}\left(\hat{s}_{t}-\frac{Q \ell^{+}}{m_{J}}, \Gamma, \mu\right) \\
& \times \tilde{B}_{-}\left(\hat{s}_{\bar{t}}-\frac{Q \ell^{-}}{m_{J}}, \Gamma, \mu\right) S_{\mathrm{hemi}}\left(\ell^{+}, \ell^{-}, \mu\right) .
\end{aligned}
$$

In terms of $M_{t}$ and $M_{\bar{t}}$ the variables $\hat{s}_{t, \bar{t}}$ are

$$
\hat{s}_{t}=2 M_{t}-2 m_{J}, \quad \hat{s}_{\bar{t}}=2 M_{\bar{t}}-2 m_{J},
$$

up to small $\Gamma / m$ power corrections. In Eqs. (114)-(116) the jet hemisphere invariant masses are $M_{t}$ and $M_{\bar{t}}$ and the short-distance top quark mass that we wish to measure is $m_{J}$. In $d^{2} \sigma / d M_{t} d M_{\bar{t}}$ the function $F$ dominates the spectrum, while $4 M_{t} M_{\bar{t}} \sigma_{0}^{H} /\left(m_{J} \Gamma\right)^{2}$ acts as a normalization constant (since $M_{t} M_{\bar{t}}$ is essentially constant in the peak region of interest). A measurement of the normalization is not optimal for determining $m_{J}$; it only has logarithmic dependence on the short-distance mass, and has larger theoretical uncertainties. On the other hand, the spectrum is very sensitive to $m_{J}$, so henceforth we focus on $F\left(M_{t}, M_{\bar{t}}, \mu\right)$.

From Eq. (115) $F$ is given by the convolution of the computable $\tilde{B}_{ \pm}$functions, with a nonperturbative hemisphere soft function, $S_{\text {hemi }}$, that describes soft final-state radiation. The majority of the important features of Eq. (115) can be explained without discussing perturbative corrections, so we focus here on the leading order result. From Eq. (96), $\tilde{B}_{ \pm}$are simply Breit-Wigner functions at leading order,

$$
\begin{aligned}
\tilde{B}_{+}\left(\hat{s}_{t}\right) & =\frac{1}{\pi\left(m_{J} \Gamma\right)} \frac{1}{\left(\hat{s}_{t} / \Gamma\right)^{2}+1}, \\
\tilde{B}_{-}\left(\hat{s}_{\bar{t}}\right) & =\frac{1}{\pi\left(m_{J} \Gamma\right)} \frac{1}{\left(\hat{s}_{\bar{t}} / \Gamma\right)^{2}+1} .
\end{aligned}
$$

For our numerical analysis we use the two-loop standard model prediction for the top width $\Gamma=1.43 \mathrm{GeV}$ [79] and we take the short-distance jet mass to be fixed at $m_{J}=$ $172 \mathrm{GeV}$. As demonstrated in Secs. II and III, $S_{\text {hemi }}$ is the same function that controls the soft radiation for massless dijets, which was studied in Refs. [32,33,56]. Hence, it is convenient for our analysis to adopt the model used to fit the massless dijet data [56],

$$
\begin{aligned}
S_{\text {hemi }}^{\mathrm{M} 1}\left(\ell^{+}, \ell^{-}\right)= & \theta\left(\ell^{+}\right) \theta\left(\ell^{-}\right) \frac{\mathcal{N}(a, b)}{\Lambda^{2}}\left(\frac{\ell^{+} \ell^{-}}{\Lambda^{2}}\right)^{a-1} \\
& \times \exp \left(\frac{-\left(\ell^{+}\right)^{2}-\left(\ell^{-}\right)^{2}-2 b \ell^{+} \ell^{-}}{\Lambda^{2}}\right) .
\end{aligned}
$$

Here the normalization constant $\mathcal{N}(a, b)$ is defined so that $\int d \ell^{+} d \ell^{-} S\left(\ell^{+}, \ell^{-}\right)=1$, the parameter $\Lambda \sim \Lambda_{\mathrm{QCD}}$ sets the scale for $\ell^{ \pm}$and hence the soft radiation, and the parameter $a$ controls how fast the soft function vanishes at the origin. The dimensionless parameter $b>-1$ controls the correlation of energy flow into the two hemispheres. Any $b \neq 0$ implies cross talk between the two hemispheres. A fit to the heavy-jet mass distribution using $e^{+} e^{-}$dijet data from LEP and SLD with $Q=m_{Z}$ gives [56]

$$
a=2, \quad b=-0.4, \quad \Lambda=0.55 \mathrm{GeV}
$$

These values were shown to yield accurate predictions for the heavy-jet mass and $C$-parameter event shapes for a wide range of energies, $Q=35-189 \mathrm{GeV}$ [56], as well as available thrust distributions with $Q=14-161 \mathrm{GeV}$ [33]. We adopt Eq. (119) as the central values for our analysis, but will also discuss how our predictions vary with changes to these model parameters.

In Fig. 7 we plot $F\left(M_{t}, M_{\bar{t}}\right)$ using Eqs. (117)-(119) and taking $Q=745 \mathrm{GeV}$. The key feature to note is that the observed peak position is not given by the short-distance top quark mass $m_{J}$ but is instead shifted upward by $\simeq 1.5 \mathrm{GeV}$. The positive sign of this shift is a prediction of Eq. (115) irrespective of the choice of parameters. The precise value for this shift depends on $Q / m_{J}, \Gamma$, as well as the parameters of the soft function. A less obvious feature of Fig. 7 is that the width of the observed peak has also increased beyond the width $\Gamma$ of Eq. (117). Physically, the reason for this behavior is that soft radiation contributes to the invariant masses, while the Breit-Wigner function is only a leading order approximation for the spectrum of the top quark and accompanying collinear gluons. Thus the arguments of $\tilde{B}_{ \pm}$in Eq. (115) subtract the dominant soft 


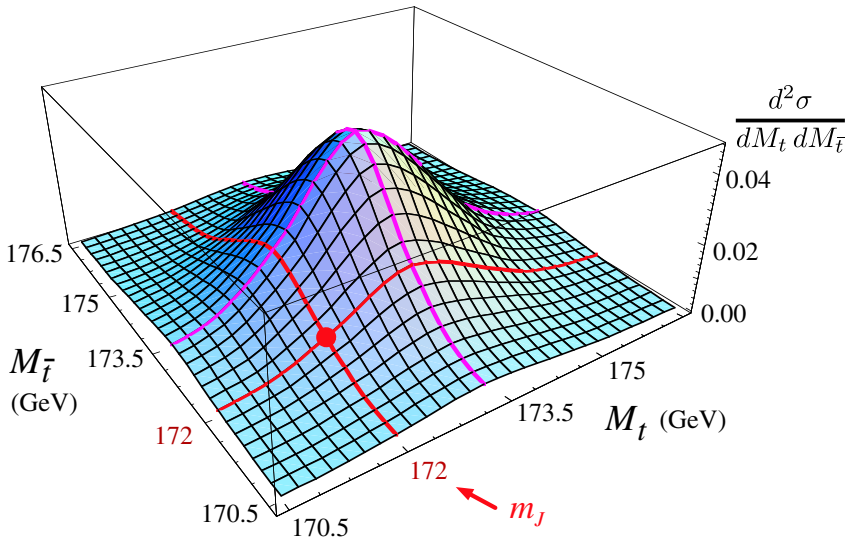

FIG. 7 (color online). Plot of $F\left(M_{t}, M_{\bar{t}}\right)$, which is the double differential hemisphere invariant mass cross section $d^{2} \sigma / d M_{t} d M_{\bar{t}}$ in units of $4 \sigma_{0}^{H} / \Gamma^{2}$. The observed peak position (intersection of the magenta lines) is not given by the true top quark mass, $m=m_{J}=172 \mathrm{GeV}$ (red lines). This peak shift depends on the energy $Q$, the width $\Gamma$, and the soft radiation function. The result is shown for $Q / m_{J}=4.33$ and the parameters in Eq. (119).

momentum component from $\hat{s}_{t, \bar{t}}$. If we approximate $S_{\text {hemi }}\left(\ell^{+}, \ell^{-}\right)$as a very narrow Gaussian centered at $\ell^{ \pm}=$ $\ell_{0}^{ \pm}$, then the observed peak simply occurs at $M_{t, \bar{t}} \sim m_{J}+$ $Q \ell_{0}^{ \pm} /\left(2 m_{J}\right)$. Although this model is too naive, we demonstrate in the next section that the linear dependence of the peak shift on $Q / m_{J}$ is in fact generic and independent of the soft function parameters. The peak width also increases linearly with $Q / m_{J}$.

The presence of the shift is due to the inclusion of soft radiation in the definition of the invariant masses $M_{t}$ and $M_{\bar{t}}$. Although we adopted a hemisphere mass definition, the same type of shift will be present for any jet algorithm that groups all the soft radiation into the jets identified for the top and antidecay products, as we discuss in Sec. V. The numerical analysis performed in this section applies equally well to these situations, though the appropriate definition and model for the soft functions $S$ for such analyses will in general be different than that in Eq. (118) with Eq. (119). We are not aware of studies where models for such soft functions were discussed.

It is important to emphasize that the shift of the observed peak position away from $m_{J}$ is not an artifact of the mass scheme. At the order used to make Fig. 7 we could set $m_{J}=m^{\text {pole }}$ since as explained in Sec. III G they differ by $\mathcal{O}\left(\alpha_{s} \Gamma\right){ }^{7}$ In a generic short-distance top quark jet-mass scheme there is a small shift $\sim \alpha_{s} \Gamma$ in the peak position due to perturbative corrections in the matrix element defining $\tilde{B}_{ \pm}$(as discussed in detail in Ref. [66]). In Sec. III G we

\footnotetext{
${ }^{7}$ In general use of $m^{\text {pole }}$ is not a good idea, since in fits it would induce an unphysical change in the required parameters $a, b, \Lambda$ order-by-order in perturbation theory
}

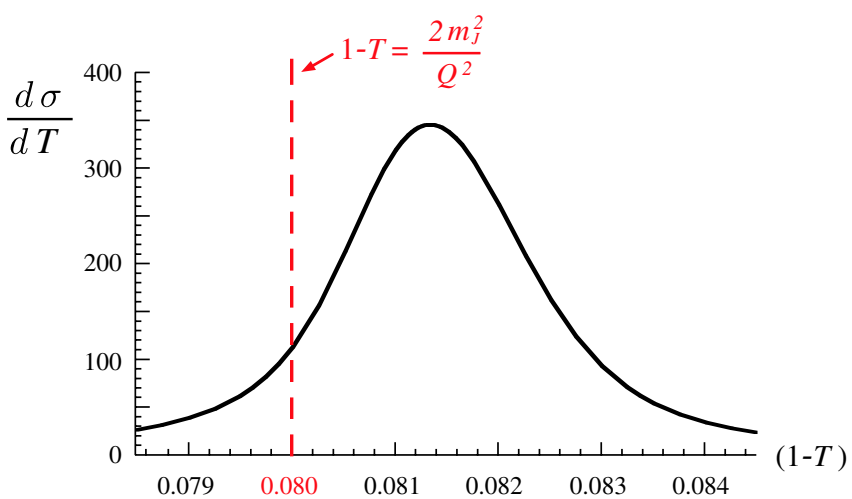

FIG. 8 (color online). Plot of the thrust distribution, $d \sigma / d T$ in units of $\sigma_{0}^{H}$, for top initiated events in the peak region. We use $Q / m_{J}=5, m_{J}=172 \mathrm{GeV}$ and the soft function parameters in Eq. (119).

defined $m_{J}$ using a jet-mass scheme which keeps the peak of $\tilde{B}_{ \pm}$fixed order-by-order in perturbation theory. In this scheme the shift in the peak location relative to the shortdistance mass is entirely due to the nonperturbative soft radiation.

Although $m_{J}$ is not determined by the peak position, the shape of the cross section is very sensitive to $m_{J}$, and hence for $\delta m_{t} \lesssim 1 \mathrm{GeV}$ the top quark mass should be determined by a fit to $F$ in Eq. (114). In Sec. III I factorization theorems for related event-shape variables were derived, including thrust $d \sigma / d T$, and the heavy-jet mass $d \sigma / d \rho$. These event shapes also exhibit a peak. They are sensitive to the top quark mass parameter $m_{J}$, and can be used for top-mass measurements. As an example, in Fig. 8 we plot $d \sigma / d T$ using $Q / m_{J}=5, m_{J}=172 \mathrm{GeV}$, and the parameters in Eq. (119). The expected peak in the thrust distribution is at $1-T \simeq 2 m^{2} / Q^{2}=0.08$ and is shifted to the right by $\Delta(1-T)=1.3 \times 10^{-3}$ by the soft radiation. Again the direction of the shift is a prediction, but the precise amount of the shift depends on the soft model parameters in Eq. (119) as well as $Q / m_{J}$. An analysis of any other event-shape distributions that are related to $d^{2} \sigma / d M_{t}^{2} d M_{\bar{t}}^{2}$ can be made in a similar fashion.

In Sec. IV B we explore the functional dependence of the peak shift for $d^{2} \sigma / d M_{t} d M_{\bar{t}}$ in greater detail. In Sec. IV C we discuss the implications of our results for fits to determine the short-distance mass.

\section{B. Analysis of the peak shift and broadening}

In this section we analyze the parameter dependence of the peak shift and broadening of the width and demonstrate that they have a linear dependence on $Q$. The main analysis is carried out assuming that the soft function model parameters have been determined from massless jet observables with small uncertainties and adopting the parameters in Eq. (119). It is, however, also instructive to study the dependence of the invariant mass distribution on variations of the model parameters, anticipating that the soft function 

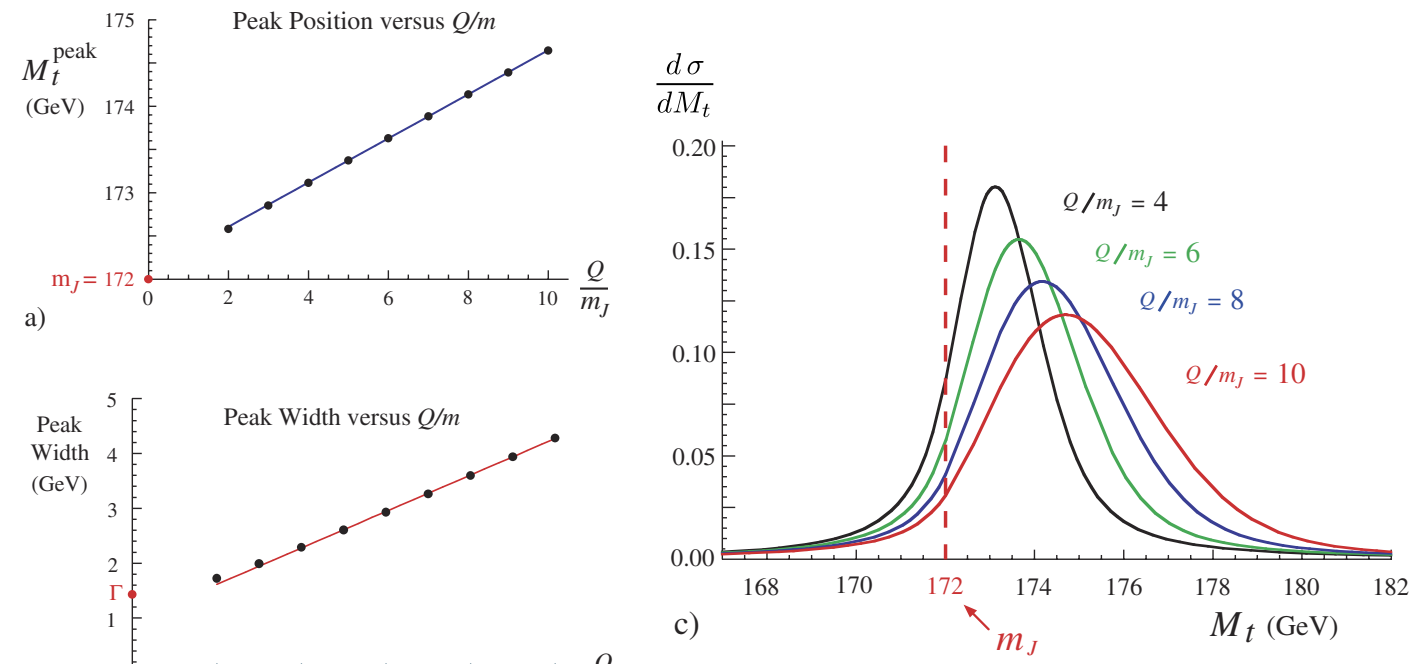

b)

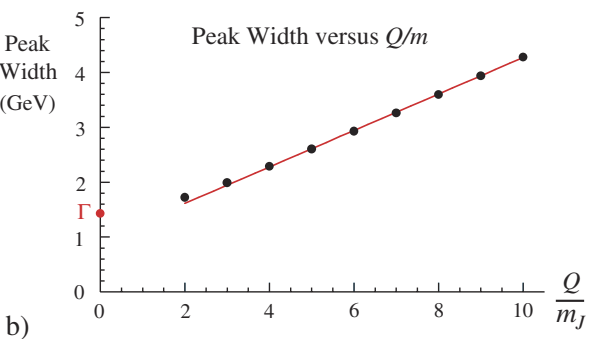

FIG. 9 (color online). Effect of a change in $Q$ on the invariant mass distribution. Results on the left are generated from $d^{2} \sigma / d M_{t} d M_{\bar{t}}$, (a) shows the peak position versus $Q / m_{J}$ and (b) gives the full width at half max versus $Q / m_{J}$. In (c) we show $d \sigma / d M_{t}$ in units of $2 \sigma_{0}^{H} / \Gamma$ for different values of $Q / m_{J}$. The curves use $m_{J}=172 \mathrm{GeV}, \Gamma=1.4 \mathrm{GeV}$, and the parameters in Eq. (119).

is different when the definition of the invariant masses is modified (see the discussion in Sec. V). We carry out such an analysis near the end of this section.

In Fig. 9(a) we plot the peak location, $M_{t}^{\text {peak }}$, for nine values of $Q . M_{t}^{\text {peak }}$ is obtained from the two-dimensional distribution, and corresponds to the intersection of the magenta lines in Fig. 7. Since $d^{2} \sigma / d M_{t} d M_{\bar{t}}$ is symmetric the value of $M_{\bar{t}}^{\text {peak }}$ is the same. Note that for $Q \simeq 2 m_{J}$ where the tops are near $t \bar{t}$ production threshold, our effective theory expansions do not apply. The straight blue line in Fig. 9(a) is a linear fit to the points with $Q / m_{J} \geq 4$, and clearly shows that the peak location grows linearly with $Q$. In Fig. 9(b) we plot the "Peak Width," defined as the full width at half max of $d^{2} \sigma / d M_{t} d M_{\bar{t}}$ in the top-variable $M_{t}$, while fixing the antitop $M_{\bar{t}}=M_{\bar{t}}^{\text {peak }}$. The red solid line is a linear fit for $Q / m_{J} \geq 4$. This figure demonstrates that we also have linear growth with $Q$ for the width of the measured invariant mass distribution. Note that the values for the peak position and peak width shown are consistent with our power counting since $\hat{s}_{t, \bar{t}}$ can be order $\Gamma$ as well as greater than $\Gamma$.

To get a better picture of how the distribution changes with $Q$ we plot the single invariant mass distribution $d \sigma / d M_{t}$ in Fig. $9(\mathrm{c})$. In particular we plot

$$
F_{1}\left(M_{t}\right)=\frac{2}{\Gamma} \int_{M_{\text {lower }}}^{M_{\text {upper }}} d M_{\bar{t}} F\left(M_{t}, M_{\bar{t}}\right)
$$

which gives $d \sigma / d M_{t}$ in units of $2 \sigma_{0}^{H} / \Gamma$. In the numerical analysis we center the integration interval $\left[M_{\text {lower }}, M_{\text {upper }}\right]$ on $M_{\bar{t}}^{\text {peak }}$ with a size that is twice the measured peak width. Hence the size of the interval depends on $Q$, but keeps the number of events collected at each $Q$ approximately constant for the comparison. For different choices of $Q$ we find that the peak position and width of $F_{1}\left(M_{t}\right)$ behave in an identical manner to Figs. 9(a) and 9(b) including having essentially the same slopes. In order to keep the area under the curves constant the peak height drops as $Q$ is increased. Note that for values $Q / m_{J} \simeq 8-10$ the observed peak location may be as much as $2.0-2.5 \mathrm{GeV}$ above the value of the Lagrangian mass $m_{J}$ one wants to measure. In our analysis $m_{J}$ is held fixed as shown by the dashed line in Fig. 9(c).

To gain an analytic understanding of this linear behavior we consider the effect of $Q$ on the mean of the cross section, which is a good approximation to the peak location. Taking the first moment with respect to $\hat{s}_{t} / 2=\left(M_{t}-\right.$ $\left.m_{J}\right)$ over an interval of size $2 L \gg Q \Lambda$ and the zeroth moment in $\hat{s}_{\bar{t}} / 2=\left(M_{\bar{t}}-m_{J}\right)$ gives

$$
\begin{aligned}
F^{[1,0]} & \equiv \frac{1}{m_{J}^{2} \Gamma^{2}} \int_{-L}^{L} d s_{t} \frac{\hat{s}_{t}}{2} \int_{-\infty}^{\infty} d s_{\bar{t}} F\left(M_{t}, M_{\bar{t}}\right)=\int_{-\infty}^{\infty} d \ell^{+} \int_{-L}^{L} d s_{t} \frac{\hat{s}_{t}}{2} \tilde{B}_{+}\left(\hat{s}_{t}-\frac{Q \ell^{+}}{m_{J}}\right) \int_{-\infty}^{\infty} d \ell^{-} S_{\mathrm{hemi}}\left(\ell^{+}, \ell^{-}\right) \\
& \simeq \frac{1}{2} \int_{-\infty}^{\infty} d \ell^{+} \int_{-L}^{L} d s_{t}\left(\hat{s}_{t}+\frac{Q \ell^{+}}{m_{J}}\right) \tilde{B}_{+}\left(\hat{s}_{t}\right) \int_{-\infty}^{\infty} d \ell^{-} S_{\mathrm{hemi}}\left(\ell^{+}, \ell^{-}\right)=\frac{Q}{2 m_{J}} S_{\mathrm{hemi}}^{[1,0]} .
\end{aligned}
$$

Thus the mean grows linearly with $Q / m_{J}$ with a slope determined by the first moment of the soft function, 
$S_{\text {hemi }}^{[1,0]}=\int d \ell^{+} d \ell^{-} \ell^{+} S_{\text {hemi }}\left(\ell^{+}, \ell^{-}\right)$. In the first equality of Eq. (121) the $\tilde{B}_{-}$function drops out because we integrate over all $\hat{s}_{\bar{t}}$. The approximation in Eq. (121) is that terms of $\sim 1 / L$ are dropped. We can also directly consider the location of the peak in $M_{t}$, again integrating over $M_{\bar{t}}$ for convenience. We use the fact that the tree level $\tilde{B}_{+}\left(\hat{s}_{t}\right)$ is symmetric and solve for $M_{t}^{\text {peak }}=m_{J}+\hat{s}_{t}^{\text {peak }} / 2$ by setting

$$
\begin{aligned}
0 & =\frac{1}{m_{J}^{2} \Gamma^{2}} \int_{-\infty}^{\infty} d \hat{s}_{\bar{t}} \frac{d F\left(M_{t}, M_{\bar{t}}\right)}{d \hat{s}_{t}}=\int_{-\infty}^{\infty} d \ell^{+} \tilde{B}_{+}^{\prime}\left(\hat{s}_{t}-\frac{Q \ell^{+}}{m_{J}}\right) \int_{-\infty}^{\infty} d \ell^{-} S_{\text {hemi }}\left(\ell^{+}, \ell^{-}\right) \\
& =\int_{-\infty}^{\infty} d \ell^{+}\left[\left(\hat{s}_{t}-\frac{Q \ell^{+}}{m_{J}}\right) \tilde{B}_{+}^{\prime \prime}(0)+\frac{1}{3 !}\left(\hat{s}_{t}-\frac{Q \ell^{+}}{m_{J}}\right)^{3} \tilde{B}_{+}^{(4)}(0)+\ldots\right] \int_{-\infty}^{\infty} d \ell^{-} S_{\mathrm{hemi}}\left(\ell^{+}, \ell^{-}\right) .
\end{aligned}
$$

For $Q \Lambda \gg m \Gamma$ we can keep only the first term which yields

$$
M_{t}^{\text {peak }} \simeq m_{J}+\frac{Q}{2 m_{J}} S_{\text {hemi }}^{[1,0]} .
$$

Thus we find the same shift as for the moment in Eq. (121). Our default model in Eq. (119) gives $S_{\text {hemi }}^{[1,0]} / 2=0.31 \mathrm{GeV}$ for the slope in $Q / m_{J}$. This can be compared with the fit to the two-dimensional peak position, Fig. 9(a), which gives a slope of $0.26 \mathrm{GeV}$. The fit to the peak position of $F_{1}\left(M_{t}\right)$ in 9(c) has a similar slope, $0.25 \mathrm{GeV}$. Finally, the first moments of $F_{1}\left(M_{t}\right)$ also display linear behavior in $Q / m_{J}$ with a slope of $0.28 \mathrm{GeV}$. We see that $S_{\text {hemi }}^{[1,0]} / 2$ accounts for the largest portion of these slopes, with the remainder being accounted for by other moments. Note that the linear behavior in $Q / m_{J}$ observed in Fig. 9 is much more accurate than the statement that $S_{\text {hemi }}^{[1,0]} / 2$ determines the proper slope at lowest order. We want to point out that a first measurement of the short-distance mass could be made using a couple of different $Q$ values and a simple extrapolation with Eq. (123).

Finally we consider the effect on the invariant mass shift from a scan over model parameters. $F\left(M_{t}, M_{\bar{t}}\right)$ depends on the parameters
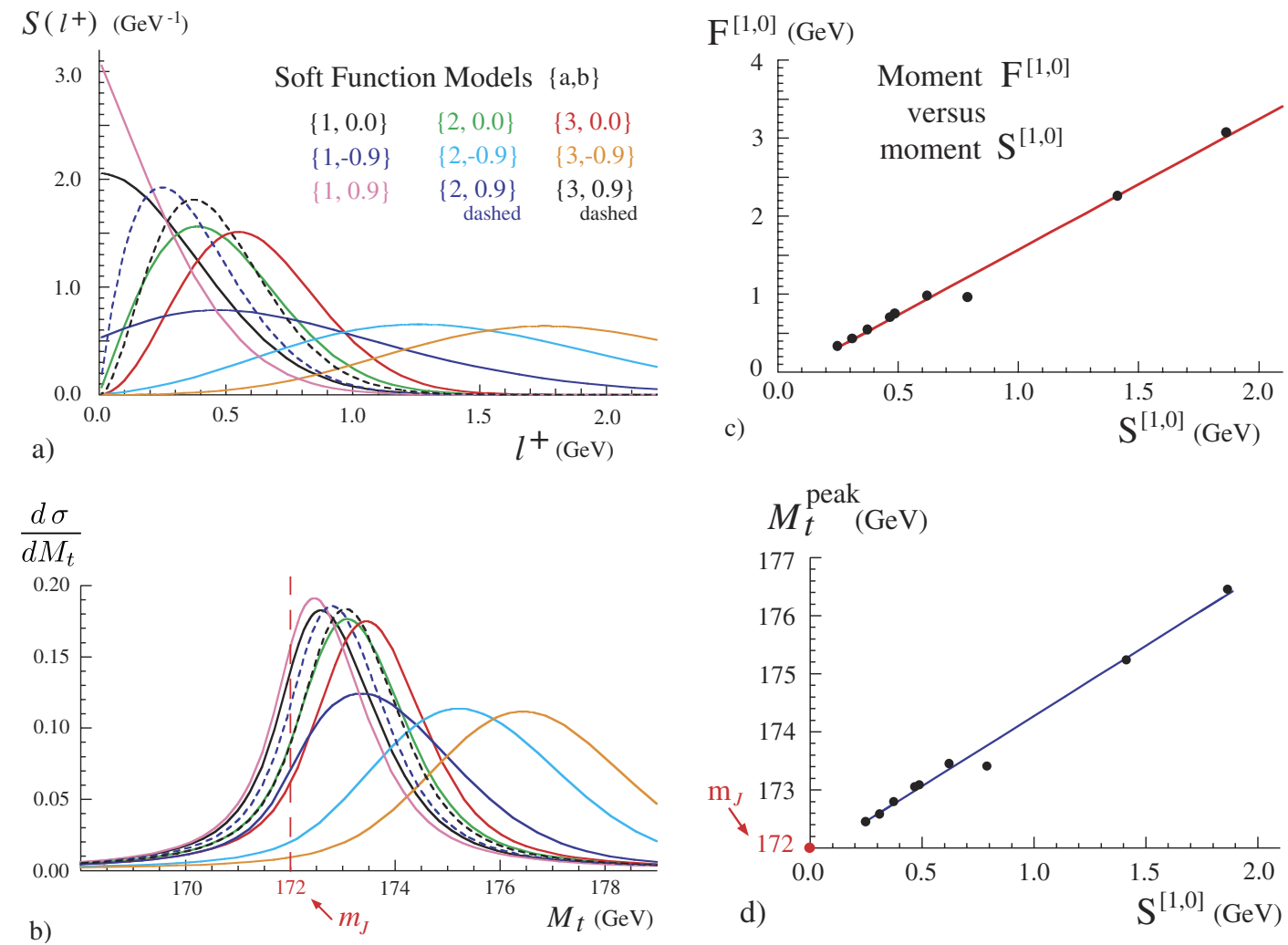

FIG. 10 (color online). Dependence of the invariant mass distribution on the soft function model parameters. In (a) we show nine models with different $a$ and $b$ parameters, and in (b) we show the resulting $d \sigma / d M_{t}$ in units of $2 \sigma_{0}^{H} / \Gamma$. In (c) we plot the first moment of the invariant mass distribution, $F^{[1,0]}$, versus the first moment of the soft function $\ell^{+}, S^{[1,0]}$. In (d) we plot the peak position of $d \sigma / d M_{t}$ versus $S^{[1,0]}$. These plots are made with $Q / m_{J}=5$ and $m_{J}=172 \mathrm{GeV}$. Note that these scans are only relevant experimentally if the universality of $S_{\text {hemi }}$ with massless dijet events is not used to determine the parameters. 


$$
m_{J}, \quad \Gamma, \quad \beta=\frac{Q \Lambda}{m_{J} \Gamma}, \quad a, \quad b .
$$

Here the scale $\Lambda$ for the soft function only shows up along with $Q / m_{J}$ in the effective boost parameter $\beta$. To demonstrate that it is $\beta$ that appears in $F\left(M_{t}, M_{\bar{t}}\right)$, switch integration variables to $x=\ell^{+} / \Lambda$ and $y=\ell^{-} / \Lambda$, and let $\hat{s}_{t, \bar{t}}=z_{t, \bar{t}} \Gamma$. This yields a soft function $\Lambda^{2} S_{\text {hemi }}(\Lambda x, \Lambda y)$ that is independent of $\Lambda$, and $\tilde{B}_{+}\left(\hat{s}_{t}-Q \ell^{+} / m_{J}\right)=$ $\tilde{B}_{+}\left(\Gamma\left(z_{t}-\beta x\right)\right)$ which is only a function of $\left(z_{t}-\beta x\right)$ times $\left(\Gamma m_{J}\right)^{-1}$. Hence $F\left(M_{t}, M_{\bar{t}}\right)$ is only a function of $\beta$, $z_{t, \bar{t}}=\left(M_{t, \bar{t}}-m_{J}\right) / \Gamma,\left(m_{J} \Gamma\right)$, and the model parameters $a$ and $b$. Hence changing $\Lambda$ has the same effect as changing $Q / m_{J}$. Below we will only consider variations of the model parameters $a$ and $b$, while keeping $\Lambda=0.55 \mathrm{GeV}$.

We generate nine soft function models from the intersection of $a=\{1,2,3\}$ and $b=\{-0.9,0.0,0.9\}$, and in Fig. 10(a) give the profile of these models by plotting $S\left(\ell^{+}\right)=\int d \ell^{-} S_{\text {hemi }}\left(\ell^{+}, \ell^{-}\right)$. Increasing $a$ shifts the distribution to larger average momenta. For each model the result for the single invariant mass distribution $F_{1}\left(M_{t}\right)$ is shown in Fig. 10(b) with curves of a matching color. We again take the $M_{\overline{\mathrm{t}}}$ integration interval, $\left[M_{\text {lower }}, M_{\text {uppper }}\right]$, to be centered on the measured peak, with a size that is twice the measured peak width. The peak positions for $F_{1}\left(M_{t}\right)$ are ordered in the same manner as the peak positions for the $S\left(\ell^{+}\right)$models. We note that models with $b=0,0.9$ generate smaller peak shifts than those with $b \sim-0.9$. To examine the peak shifts more quantitatively we plot the first moment $F^{[1,0]}$ versus the first moment $S_{\text {hemi }}^{[1,0]}$ in Fig. 10(c). To compute $F^{[1,0]}$ we restrict the two integrals in Eq. (121) to the same interval choice $\left[M_{\text {lower }}, M_{\text {upper }}\right]$. From the figure we observe that the mean of the invariant mass distributions for different models falls close to a straight line. In Fig. 10(d) we plot the peak position $M_{t}^{\text {peak }}$ for each model versus the first moment $S_{\text {hemi }}^{[1,0]}$ and observe that the behavior is also quite linear. We conclude that the main effects of $a, b$ on the peak shift are controlled by the first-moment parameter $S_{\text {hemi }}^{[1,0]}$.

\section{Implications for top quark mass measurements}

In this section we take a step back to consider the more general implications of our method for top quark mass measurements. In a realistic top quark mass analysis at a hadron collider with $p p$ or $p \bar{p}$ collisions, the set of issues that effect the accuracy for a $m_{t}$ measurement and that can potentially be improved by theoretical progress includes (i) the choice of the observable to be measured, (ii) the topmass definition, (iii) hadronization effects, (iv) color reconnection, (v) final-state radiation, (vi) initial state radiation, (vii) underlying events, (viii) cuts to remove the beam remnant, and (ix) parton distribution functions. In our analysis we treat $e^{+} e^{-}$collisions which allows us to investigate strong interaction effects in categories (i) $-(\mathrm{v})$.
We briefly discuss what our result for $d^{2} \sigma / d M_{t} d M_{\bar{t}}$ implies for these uncertainties.

The main advantage of the factorization approach is that it keeps careful track of how changing the observable affects corrections from the other categories. For example, switching from invariant mass variables to thrust gives a different function for the nonperturbative soft radiation, but the soft functions in these observables are related by Eq. (110), and one model can be used to fit both of them. In our analysis, the inclusive nature of the hemisphere invariant mass observable reduces the uncertainty from hadronization effects. In particular it yields jet functions which sum over hadronic states with invariant mass up to $\sim m \Gamma$, and remain perturbatively computable due to the lowmomentum cutoff provided by the top width. Final-state gluon radiation from the decay products contributes to the width, while emissions of soft gluons in the c.m. frame organize themselves with radiation from the top quark to give a single universal soft radiation function. Thus, nontrivial color reconnection effects between the decay products are power suppressed. The level of control provided by the factorization theorem therefore provides a significant reduction in the associated uncertainties. Of course the nature of this control is observable dependent and will undoubtedly change in the hadronic collider environment, in particular, hemisphere masses are not suitable for mass measurements at the Tevatron or LHC. Nevertheless, we expect the control provided by the factorization approach to provide useful applications to this case as well. Finally, as discussed in detail in Sec. III G, the inherent theoretical ambiguity in the pole mass, $\delta m^{\text {pole }} \sim \Lambda_{\mathrm{QCD}}$, can be avoided by switching to a short-distance jet mass $m_{J}$. This mass definition is suitable for reconstruction measurements in $e^{+} e^{-}$collisions, and in principle also for $p \bar{p}$ and $p p$ collisions.

For the case studied in detail here, a measurement of $d^{2} \sigma / d M_{t} d M_{\bar{t}}$ from energetic top jets in $e^{+} e^{-}$collisions, there are at least two ways the result in Eq. (114) can be used to fit for the short-distance mass $m_{J}$. In the first one takes the soft function model parameters $a, b$, and $\Lambda$ from a fit to massless dijet event shapes, and then analyzes $d^{2} \sigma / d M_{t} d M_{\bar{t}}$ to fit for $m_{J}$. This method makes use of the universality of the soft hemisphere function $S_{\text {hemi }}\left(\ell^{+}, \ell^{-}\right)$ between massive and massless jets. Alternatively, one can vary $Q$ and do a simultaneous fit to $m_{J}, a, b$, and the effective boost parameter $\beta$, to determine the soft parameters from the same data used to determine $m_{J}$. This may be advantageous if the energy resolution, jet energy scale, or other experimental effects have nontrivial interactions with the soft radiation that are particular to $t \bar{t}$ decays. In the next section we consider how the factorization theorem is modified by the use of an inclusive $k_{T}$ algorithm rather than using hemisphere masses.

To conclude this section we note that in our analysis we have not accounted for QED effects since in this work we 
are interested in treating the effects of the strong interactions. For a realistic description of experimental data obtained at a future linear collider QED effects will of course have to be included. Effects from QED can contribute to the categories (ii), (v), (vi) as well as to the QED analogues of categories (iv) and (ix). As such the treatment of these QED effects is straightforward and can be included naturally in our factorization approach. To be more concrete, the QED analogue to parton distribution functions entails to account for initial state radiation, beam strahlung and the beam energy spread through a luminosity spectral function which has to be convoluted with the QCD cross section. The luminosity spectrum is obtained from analyzing Babbha scattering [80]. The effect of soft photons showing up in the two hemispheres can be incorporated as additional perturbative contributions in the soft function, and the effects of collinear photons can be incorporated as additional perturbative contributions to the jet functions in our factorization theorem. Finally, the effect of hard photons not aligned with the top and antitop jets is analogous to the productions of additional hard jets, which leads to contributions in the hemisphere masses away from the resonance region. Compared to the QCD effects treated in this work the above QED corrections lead to changes of the invariant mass distribution that are suppressed by the small QED coupling.

\section{FACTORIZATION FOR MASSES BASED ON JET ALGORITHMS}

In this work we have defined the top and antitop invariant masses up to now as the invariant masses of all particles in the two hemispheres defined through the thrust axis of each event, see Fig. 3. In past experimental studies, on the other hand, a $k_{T}$ algorithm was employed so that each event results in exactly six jets for the all-hadronic decay mode, $e^{+} e^{-} \rightarrow t \bar{t}+X \rightarrow 6$ jets [8,9]. Of these six jets, three jets were combined to the top and the other three to the antitop invariant mass. We remind the reader that jet algorithms for $e^{+} e^{-}$collisions do not need to remove any "beam remnants," so every final-state particle of an event is eventually either assigned to the top or the antitop invariant mass. It is this crucial aspect of jet algorithms for $e^{+} e^{-}$collisions that makes them share a number of important properties with the hemisphere invariant masses that we have analyzed so far. One of these properties is that having both invariant masses in the peak region close to the top quark mass automatically ensures that the event is dijetlike, such that the EFT setup discussed in the previous sections can be applied in the same way as it was for the hemisphere masses.

In this section we show that using a jet algorithm with the property mentioned above for the top and antitop invariant mass reconstruction, the double differential top and antitop invariant mass distribution in the peak region can be written in the factorized form of Eq. (104), but with a different soft function $S\left(\ell^{+}, \ell^{-}\right)$which depends on the jet algorithm. All other ingredients, the jet functions $B_{ \pm}$and the matching and evolution factors, are identical. For the proof we assume that the top and antitop decay jets ${ }^{8}$ obtained from the jet algorithm can be assigned unambiguously to the top and the antitop, i.e. we neglect the combinatorial background. This simplification is possible at leading order in $m / Q$ because hard jets from the top decay only have a very small probability of order $(\mathrm{m} / Q)^{2}$ to show up in the hemisphere of the antitop quark, as was already pointed out in Sec. III E. The analogous statement is of course also true for hard jets from the antitop decay. Moreover we assume that the jet algorithm uses simple addition of four-vectors as its recombination scheme for merging final-state objects.

The proof can be carried out in the EFT setups described in Sec. IIIF and Fig. 5. The crucial point that has to be shown is that, at leading order in the power counting, the total $n$-collinear momentum $P_{X_{n}}$ enters exclusively the top invariant mass, while the total $\bar{n}$-collinear momentum enters exclusively the antitop invariant mass, just as for the hemisphere mass definitions explained in Sec. IIID. Furthermore, the prescription to determine the soft function has to be provided for a given jet algorithm. This corresponds to defining appropriate projection operators $\hat{P}_{a}$ and $\hat{P}_{b}$ in Eq. (74) or equivalently the momenta $k_{s}^{a}$ and $k_{s}^{b}$ for each state $\left|X_{s}\right\rangle$. Apart from that, the derivation of the factorization theorem goes along the same lines as for the hemisphere case described in detail in the previous sections.

Concerning the assignment of $n$ - and $\bar{n}$-collinear momenta it is easy to see that the top and antitop collinear momenta are attributed correctly to the top and antitop invariant masses since we can neglect combinatorial background for the assignment of top and antitop decay jets at leading order. Assuming, for example, a $k_{T}$ jet algorithm similar to Refs. [8,9] where all final-state particles are combined to exactly six jets, one can also conclude that at leading order in $m / Q$ the $n$-collinear gluons are properly assigned to the top invariant mass, since these gluons are radiated into the $n$ hemisphere and therefore assigned to one of the three hard jets from the top quark decay. The analogous conclusion, of course, also applies to the $\bar{n}$-collinear gluons. This shows that the top-mass reconstruction based on a jet algorithm treats $n$ - and $\bar{n}$-collinear momenta essentially in the same way as the hemisphere method. It also means that the double differential top and antitop invariant mass distribution based on a jet algorithm can be derived in complete analogy to the hemisphere case and has the form shown in Eq. (104). The soft function depends on the jet algorithm that is employed, and, in

\footnotetext{
${ }^{8}$ For this discussion we deal with the case that the top quarks decay all-hadronically into jets. However, our arguments also work in principle for final states with leptons plus jets.
} 
particular, on the distance measure implemented in the algorithm. Whether a soft gluon of a given energy ends up contributing to the top or the antitop invariant mass depends on its relative angles with respect to the hard jets coming from the top and antitop decay. So upon averaging over the hard jet-configurations, a soft gluon with a given energy and a given angle with respect to the thrust axis contributes either to the top or to the antitop invariant mass governed by a probability function that is determined by the jet algorithm. This means that a soft gluon in, let us say, the $n$ hemisphere has in general a nonvanishing probability to be eventually assigned to the antitop invariant mass. The equivalence of the top-down and the bottom-up approaches to the RG evolution in the EFT's used to derive the factorization theorem further ensures that the RG running of the soft function for a given jet algorithm agrees with the running of the hemisphere soft function $S_{\text {hemi }}$, although their scale-independent terms differ. (We assume that the jet algorithm is symmetric in its treatment of top and antitop final states.) The explicit one-loop expressions for the RG running and the scale-independent contributions of the soft function for a general jet algorithm and for the hemisphere masses will be given in Ref. [66].

\section{CONCLUSIONS}

The reconstruction of top quark invariant mass distributions is one of the major methods for measuring the topmass $m$ at present and future collider experiments. Using a sequence of effective theories to separate effects at different mass scales we presented an analytic factorization approach for the top invariant mass distribution in the peak region. To be definite, we derived the double differential top/antitop invariant mass distribution $d^{2} \sigma / d M_{t} d M_{\bar{t}}$ in $e^{+} e^{-}$collisions for c.m. energies $Q \gg m$, where $M_{t, \bar{t}}$ are defined as the total invariant masses of all particles in the two hemispheres determined with respect to the event thrust axis. The factorization formula is given in Eq. (104) and represents the leading order result in an expansion in $m / Q$ and $\Gamma / m$, where $\Gamma$ is the top quark total width.

The factorization formula consists of two jet functions for top and antitop quarks, which depend strongly on the top quark Lagrangian mass, and can be computed perturbatively order-by-order in $\alpha_{s}$. It also involves a nonperturbative soft function that describes the momentum distribution of soft final-state radiation. Using alternative invariant mass prescriptions, for which the soft particles are assigned differently to $M_{t}$ and $M_{\bar{t}}$, the same factorization formula applies, but with a different soft function. The observable invariant mass distribution is obtained from a convolution of the perturbative jet functions with the nonperturbative soft function. Through this convolution the energy of the maximum and the width of the observed distribution are dependent on the c.m. energy $Q$. For a lowest order analysis see Figs. 7 and 9, and the accompanying discussion.
A very important outcome of the derivation is that the soft function for the hemisphere mass prescription also governs event-shape distributions for massless dijet events for which plenty of data has been collected at LEP and previous $e^{+} e^{-}$experiments. Since the soft function can be determined from these data, it is possible to predict the top invariant mass distribution based on the hemisphere prescription as a function of the c.m. energy $Q$, the strong coupling $\alpha_{s}$, and the Lagrangian top mass in different mass schemes without hadronization uncertainties at leading order in the expansion in $m / Q, \Gamma / m$, and $\Lambda_{\mathrm{QCD}} / m$. In principle, this allows one to measure a short-distance top quark mass from reconstruction with a precision better than $\Lambda_{\mathrm{QCD}}$.

We have proposed a new short-distance mass scheme called top quark jet mass which can be measured with minimized theoretical uncertainties from data obtained at a future linear collider and which can be reliably related to other known short-distance masses such as the threshold or the $\overline{\mathrm{MS}}$ masses. We also expect that, quite generally, the jet-mass scheme will provide an appropriate mass scheme for jet related observables involving heavy quarks.

The factorization approach developed in this work can be applied to determine the top quark mass from reconstruction of the top/antitop quark invariant mass distributions at a future $e^{+} e^{-}$linear collider. At present the most precise method to measure the top quark mass at a future linear collider is the threshold scan method. It relies on the determination of the hadronic $R$-ratio for c.m. energies around twice the top mass and will provide a short-distance top quark mass measurement with theoretical and experimental uncertainties at the level of $100 \mathrm{MeV}$. Compared to the measurement of the $R$-ratio for the threshold scan, the reconstruction of the top/antitop invariant mass distribution is without a doubt substantially more complicated. However, it has the advantage that it can be carried out at any c.m. energy above threshold and that substantially more luminosity can be spent on it. Given that our factorization approach gives control of the perturbative and nonperturbative effects contributing to the invariant mass distributions, we believe that it could eventually become a method that competes with the threshold scan. We also note that for an initial $500 \mathrm{GeV}$ linear collider, as planned for the ILC project, a careful analysis of subleading power corrections to the leading order factorization formulas derived in this work is mandatory. It is straightforward to use the effective theory framework developed in this work to account for these power corrections.

The factorization ideas proposed in this work can be applied to mass distributions of other final-state particles produced in $e^{+} e^{-}$collisions in a straightforward manner. Notable examples include single top quark production, the production of $W$ bosons or of new heavy colored unstable particles such as squarks or gluinos in certain supersymmetric new physics scenarios. They will also be relevant 
for predicting invariant mass distributions at hadron colliders. However, at hadron colliders there are additional complications that still need to be resolved as discussed in Sec. IV C. These include the initial state radiation and incorporating parton distribution functions, which lead to a distribution for $Q$ and require modifications of the concept of event shapes, the large $p_{T}$ cuts needed to get clear signals away from the beam remnant, and the effects of underlying events, which need to be taken into account. Finally, the algorithm for defining and measuring the invariant mass of jets that contain the top decay products is different in the LHC environment. We plan to address these issues in future work.

\section{ACKNOWLEDGMENTS}

We would like to thank A. Juste, S. Kluth, S. Menke, and M. Wise for helpful discussions. We also thank C. Bauer and M. Dorsten for collaboration in an early stage of this work. S. F. and S. M. thank the visitor program of the MaxPlanck-Institute for Physics for support. This work was supported in part by the Offices of Nuclear and Particle Physics of the U.S. Department of Energy under DEFG02-94ER40818, DE-FG03-92ER40701, and DEFG02-06ER41449, and in part by the EU network Contract No. MRTN-CT-2006-035482 (FLAVIAnet). I. S. and S.F. were supported in part by the DOE OJI program, and I. S. was supported in part by the Sloan Foundation.
[1] S. Heinemeyer, S. Kraml, W. Porod, and G. Weiglein, J. High Energy Phys. 09 (2003) 075.

[2] E. Brubaker et al. (Tevatron Electroweak Working Group), arXiv:hep-ex/0608032.

[3] A. P. Heinson (CDF Collaboration and D0 Collaboration), AIP Conf. Proc. 870, 223 (2006).

[4] F. Abe et al. (CDF Collaboration), Phys. Rev. D 50, 2966 (1994).

[5] V. M. Abazov et al. (D0 Collaboration), Nature (London) 429, 638 (2004).

[6] I. Borjanovic et al., Eur. Phys. J. C 39S2, 63 (2005).

[7] A. I. Etienvre, Proc. Sci., TOP2006 (2006) 023.

[8] S. V. Chekanov, arXiv:hep-ph/0206264.

[9] S. V. Chekanov and V.L. Morgunov, Phys. Rev. D 67, 074011 (2003).

[10] V.S. Fadin and V. A. Khoze, JETP Lett. 46, 525 (1987) [Pis'ma Zh. Eksp. Teor. Fiz. 46, 417 (1987)].

[11] M. J. Strassler and M. E. Peskin, Phys. Rev. D 43, 1500 (1991).

[12] V. S. Fadin and V. A. Khoze, Sov. J. Nucl. Phys. 53, 692 (1991) [Yad. Fiz.53, 1118 (1991)].

[13] M. Jezabek, J. H. Kuhn, and T. Teubner, Z. Phys. C 56, 653 (1992).

[14] Y. Sumino, K. Fujii, K. Hagiwara, H. Murayama, and C. K. Ng, Phys. Rev. D 47, 56 (1993).

[15] A. H. Hoang et al., Eur. Phys. J. direct C 2, 1 (2000).

[16] A.H. Hoang, A. V. Manohar, I. W. Stewart, and T. Teubner, Phys. Rev. Lett. 86, 1951 (2001).

[17] A. H. Hoang, A. V. Manohar, I. W. Stewart, and T. Teubner, Phys. Rev. D 65, 014014 (2001).

[18] A. Pineda and A. Signer, Nucl. Phys. B762, 67 (2007).

[19] A. H. Hoang and C. J. Reisser, Phys. Rev. D 71, 074022 (2005).

[20] G. T. Bodwin, E. Braaten, and G. P. Lepage, Phys. Rev. D 51, 1125 (1995).

[21] M.E. Luke, A. V. Manohar, and I. Z. Rothstein, Phys. Rev. D 61, 074025 (2000).

[22] D. Peralta, M. Martinez, and R. Miquel, Proceedings of the 4th International Workshop on Linear Coilliders, Sitges, Barcelona, Spain, 1999.
[23] M. Martinez and R. Miquel, Eur. Phys. J. C 27, 49 (2003).

[24] M. Beneke et al., arXiv:hep-ph/0003033.

[25] C. W. Bauer, S. Fleming, and M. E. Luke, Phys. Rev. D 63, 014006 (2000).

[26] C. W. Bauer, S. Fleming, D. Pirjol, and I. W. Stewart, Phys. Rev. D 63, 114020 (2001).

[27] C. W. Bauer and I. W. Stewart, Phys. Lett. B 516, 134 (2001).

[28] C. W. Bauer, D. Pirjol, and I. W. Stewart, Phys. Rev. D 65 , 054022 (2002).

[29] A. K. Leibovich, Z. Ligeti, and M. B. Wise, Phys. Lett. B 564, 231 (2003).

[30] A. V. Manohar and M. B. Wise, Cambridge Monogr. Part. Phys., Nucl. Phys., Cosmol. 10, 1 (2000).

[31] M. Beneke, A.P. Chapovsky, A. Signer, and G. Zanderighi, Nucl. Phys. B686, 205 (2004).

[32] G.P. Korchemsky, arXiv:hep-ph/9806537.

[33] G. P. Korchemsky and G. Sterman, Nucl. Phys. B555, 335 (1999).

[34] C. W. Bauer, A. V. Manohar, and M. B. Wise, Phys. Rev. Lett. 91, 122001 (2003).

[35] C. W. Bauer, D. Pirjol, and I. W. Stewart, Phys. Rev. D 67, 071502 (2003).

[36] C. Lee and G. Sterman, Phys. Rev. D 75, 014022 (2007).

[37] S. Cortese and R. Petronzio, Phys. Lett. B 253, 494 (1991).

[38] S.S. D. Willenbrock and D. A. Dicus, Phys. Rev. D 34, 155 (1986).

[39] J. M. Butterworth, B. E. Cox, and J. R. Forshaw, Phys. Rev. D 65, 096014 (2002).

[40] W. Skiba and D. Tucker-Smith, Phys. Rev. D 75, 115010 (2007).

[41] C. W. Bauer, S. Fleming, D. Pirjol, I.Z. Rothstein, and I. W. Stewart, Phys. Rev. D 66, 014017 (2002).

[42] E. Eichten and B. Hill, Phys. Lett. B 234, 511 (1990).

[43] N. Isgur and M. B. Wise, Phys. Lett. B 232, 113 (1989).

[44] N. Isgur and M. B. Wise, Phys. Lett. B 237, 527 (1990).

[45] B. Grinstein, Nucl. Phys. B339, 253 (1990).

[46] H. Georgi, Phys. Lett. B 240, 447 (1990).

[47] M. Beneke, A.P. Chapovsky, A. Signer, and G. Zanderighi, Phys. Rev. Lett. 93, 011602 (2004). 
[48] A.H. Hoang and C. J. Reisser, Phys. Rev. D 74, 034002 (2006).

[49] W. Beenakker, F. A. Berends, and A. P. Chapovsky, Nucl. Phys. B573, 503 (2000).

[50] I. Z. Rothstein, Phys. Rev. D 70, 054024 (2004).

[51] J. Chay, C. Kim, and A. K. Leibovich, Phys. Rev. D 72, 014010 (2005).

[52] J. Chay, C. Kim, Y. G. Kim, and J.-P. Lee, Phys. Rev. D 71, 056001 (2005).

[53] C. M. Arnesen, J. Kundu, and I. W. Stewart, Phys. Rev. D 72, 114002 (2005).

[54] A. V. Manohar and I. W. Stewart, Phys. Rev. D 76, 074002 (2007).

[55] G. P. Korchemsky and G. Sterman, Nucl. Phys. B437, 415 (1995).

[56] G. P. Korchemsky and S. Tafat, J. High Energy Phys. 10 (2000) 010.

[57] C. W. Bauer, C. Lee, A. V. Manohar, and M.B. Wise, Phys. Rev. D 70, 034014 (2004).

[58] A. H. Hoang, M. C. Smith, T. Stelzer, and S. Willenbrock, Phys. Rev. D 59, 114014 (1999).

[59] M. Beneke, Phys. Lett. B 434, 115 (1998).

[60] N. Uraltsev, arXiv:hep-ph/9804275.

[61] A. H. Hoang, Z. Ligeti, and A. V. Manohar, Phys. Rev. Lett. 82, 277 (1999).

[62] C. W. Bauer, D. Pirjol, and I. W. Stewart, Phys. Rev. D 68, 034021 (2003).

[63] T. Sjostrand and V. A. Khoze, arXiv:hep-ph/9908408.
[64] V. A. Khoze, W. J. Stirling, and L.H. Orr, Nucl. Phys. B378, 413 (1992).

[65] A. V. Manohar, Phys. Rev. D 68, 114019 (2003).

[66] S. Fleming, A. H. Hoang, S. Mantry, and I. W. Stewart, arXiv:0711.2079.

[67] K. S. M. Lee and I. W. Stewart, Nucl. Phys. B721, 325 (2005).

[68] S. Catani, Y.L. Dokshitzer, M. Olsson, G. Turnock, and B. R. Webber, Phys. Lett. B 269, 432 (1991).

[69] J. M. Butterworth, J. P. Couchman, B.E. Cox, and B. M. Waugh, Comput. Phys. Commun. 153, 85 (2003).

[70] S. J. Parke and Y. Shadmi, Phys. Lett. B 387, 199 (1996).

[71] B. A. Kniehl, Phys. Lett. B 237, 127 (1990).

[72] G. J. H. Burgers, Phys. Lett. 164B, 167 (1985).

[73] A. H. Hoang, J.H. Kuhn, and T. Teubner, Nucl. Phys. B452, 173 (1995).

[74] T. Becher and K. Melnikov, J. High Energy Phys. 06 (2007) 084.

[75] J.-y. Chiu, F. Golf, R. Kelley, and A. V. Manohar, Phys. Rev. Lett. 100, 021802 (2008).

[76] M. Beneke, Phys. Rep. 317, 1 (1999).

[77] S. Fleming, A. H. Hoang, S. Mantry, and I. W. Stewart (unpublished).

[78] G. P. Korchemsky and A. V. Radyushkin, Phys. Lett. B 279, 359 (1992).

[79] A. Czarnecki and K. Melnikov, Nucl. Phys. B544, 520 (1999).

[80] S. T. Boogert and D. J. Miller, arXiv:hep-ex/0211021. 